2005-11-29

\title{
A New Method for the Rapid Calculation of Finely-Gridded Reservoir Simulation Pressures
}

\author{
Benjamin Arik Hardy \\ Brigham Young University - Provo
}

Follow this and additional works at: https://scholarsarchive.byu.edu/etd

Part of the Chemical Engineering Commons

\section{BYU ScholarsArchive Citation}

Hardy, Benjamin Arik, "A New Method for the Rapid Calculation of Finely-Gridded Reservoir Simulation Pressures" (2005). Theses and Dissertations. 712.

https://scholarsarchive.byu.edu/etd/712

This Thesis is brought to you for free and open access by BYU ScholarsArchive. It has been accepted for inclusion in Theses and Dissertations by an authorized administrator of BYU ScholarsArchive. For more information, please contact scholarsarchive@byu.edu, ellen_amatangelo@byu.edu. 


\section{A NEW METHOD FOR THE RAPID CALCULATION OF FINELY-GRIDDED RESERVOIR SIMULATION}

PRESSURES

by

Benjamin A. Hardy

A thesis submitted to the faculty of

Brigham Young University

in partial fulfillment of the requirements for the degree of

Master of Science

Department of Chemical Engineering

Brigham Young University

December 2005 
Copyright @ 2005 Benjamin A. Hardy

All Rights Reserved 
BRIGHAM YOUNG UNIVERSITY

GRADUATE COMMITTEE APPROVAL

of a thesis submitted by

Benjamin A. Hardy

This thesis has been read by each member of the following graduate committee and by a majority vote has been found satisfactory.

Date

Date

Date
Hugh B. Hales, Chair

Larry L. Baxter

Merrill W. Beckstead 


\section{BRIGHAM YOUNG UNIVERSITY}

As chair of the candidate's graduate committee, I have read the thesis of Benjamin A. Hardy in its final form and have found that (1) its format, citations, and bibliographical style are consistent and acceptable and fulfill university and department style requirements; (2) its illustrative materials including figures, tables, and charts are in place; and (3) the final manuscript is satisfactory to the graduate committee and is ready for submission to the university library.

Date

Hugh B. Hales

Chair, Graduate Committee

Accepted for the Department

William G. Pitt

Graduate Coordinator

Accepted for the College

Alan R. Parkinson
Dean, Ira A. Fulton College of Engineering and
Technology




\begin{abstract}
A NEW METHOD FOR THE RAPID CALCULATION

OF FINELY-GRIDDED RESERVOIR SIMULATION

PRESSURES
\end{abstract}

\author{
Benjamin A. Hardy \\ Department of Chemical Engineering \\ Master of Science
}

A new method for the determination of finely-gridded reservoir simulation pressures has been developed. It is estimated to be as much as hundreds to thousands of times faster than other methods for very large reservoir simulation grids. The method extends the work of Weber et al. ${ }^{27}$ Weber demonstrated accuracies for the pressure solution normally requiring millions of cells using traditional finite-difference equations with only hundreds of cells. This was accomplished through the use of finite-difference equations that incorporate the physics of the flow. Although these coarse-grid solutions achieve accuracies normally requiring orders of magnitude more resolution, their coarse resolution does not resolve local pressure variations resulting from fine-grid permeability variations. Many oil reservoir simulation models require fine grids to adequately represent the reservoir properties. Weber's coarse grids are of little value. This study 
takes advantage of the accurate coarse-grid solutions of Weber, by nesting them in the requisite fine grids to achieve much faster solutions of the large systems.

Application of the nested-grid method involved calculating an accurate solution on a coarse grid, nesting the coarse-grid solution as fixed points into a finer grid and solving. Best results were obtained when an optimal number of coarse-grid pressure points were nested into the fine grid and when an optimal number of nested-grid systems were used.

\begin{tabular}{|c|c|c|}
\hline \multicolumn{3}{|c|}{ Speed Increase Factor for Weber's Results } \\
\hline Grid Size & $10^{6}$ & $10^{9}$ \\
\hline One Optimum Nested-Grid & 115.84 & 812.98 \\
\hline Two Optimum Nested-Grids & 278.21 & 2796.81 \\
\hline Three Optimum Nested Grids & 351.32 & 3860.19 \\
\hline
\end{tabular}




\section{ACKNOWLEDGEMENTS}

I would like to express appreciation to my advisor Dr. Hugh B. Hales for his untiring support and kindness. I would also like to thank the members of my graduate committee, Dr. Larry L. Baxter and Dr. Merrill W. Beckstead for their input and willingness to be on my committee. I am very grateful for the financial support that IRSRI has provided during my time as a graduate student.

I consider myself fortunate to have had the privilege of completing my Master of Science degree at Brigham Young University among such outstanding students and faculty. 


\section{TABLE OF CONTENTS}

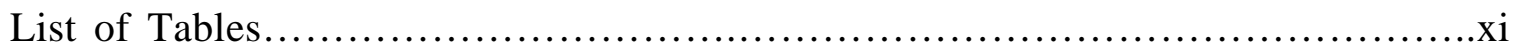

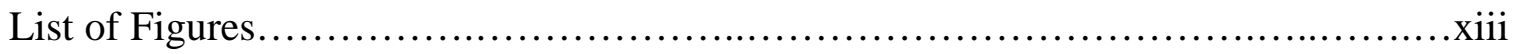

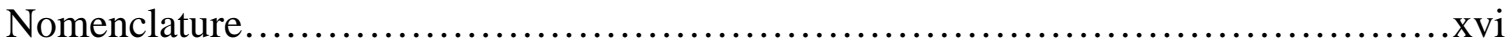

Chapter $1 \quad$ Introduction..........................................................

1.1 Technologies That Depend on Reservoir Simulation.......................2

1.2 Reservoir Descriptions.................................................

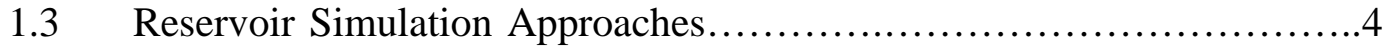

1.4 Review of Multiscale Methods........................................

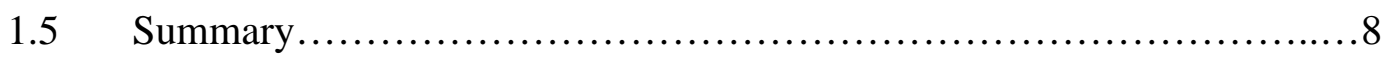

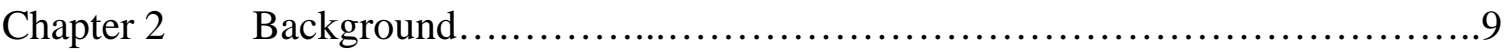

2.1 Weber's Equations and Nested-Grid Description........................9

2.2 Comparison to Multigrid Method.........................................11

2.3 Reservoir Description................................................13

2.4 Solution of the Pressure Equation.........................................14

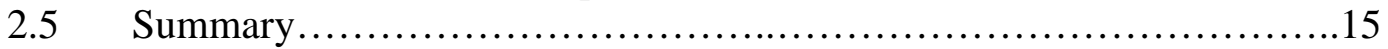

Chapter 3 Testing the Performance of Various Solvers as a Function of Grid Size Size.................................................................

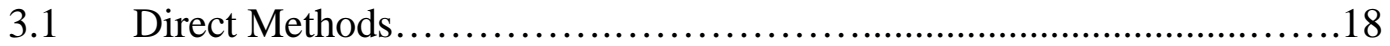

3.2 Iterative Methods..................................................18

3.3 MATLAB 7.0 Iterative Methods.........................................22

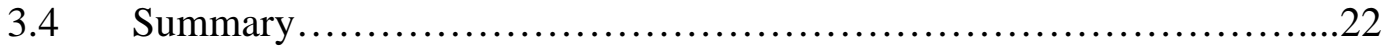

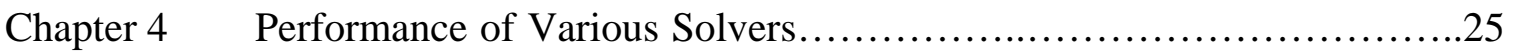

4.1 Direct Methods: Results...........................................25

4.2 Stationary and Nonstationary Iterative Methods: Results..................27

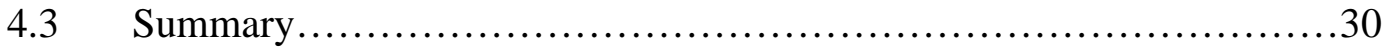

Chapter $5 \quad$ Determination of Best Solver............................................31 


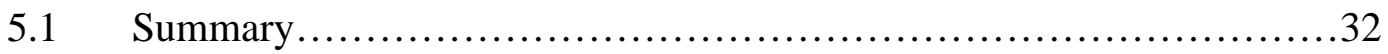

Chapter 6 Implementation of Solver in Nested-Grid Method........................33

6.1 Grid Geometries and General Setup....................................33

6.2 Calculation of Coarse-Grid Pressure..................................36

6.3 Calculation of Fine-Grid Pressure: LaPlace SOR Results..................37

6.4 Nested-Grid Method: GMRES Results..................................38

6.5 Data Analysis................................................... 40

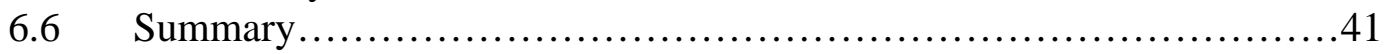

Chapter $7 \quad$ Interpolation......................................................... 43

7.1 Considering Interpolation..........................................43

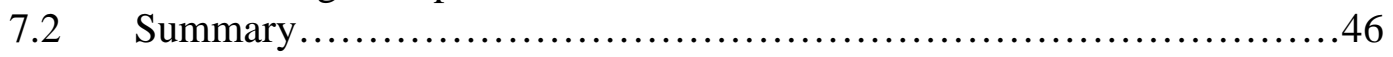

Chapter $8 \quad$ Weber's Coefficients............................................... 47

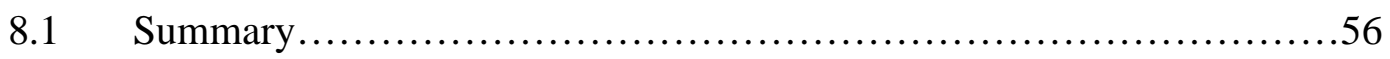

Chapter 9 Study with Weber's Coefficients Applied...............................57

9.1 Grid Geometries and General Setup.................................5 57

$9.2 \quad$ Nested-Grid Results..............................................61

9.3 Regression and Dimensionless Time Analysis.........................62

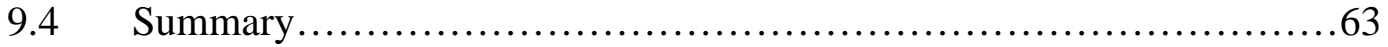

Chapter $10 \quad$ Regression of All Results...........................................65

10.1 Regression of LaPlace and Weber SOR ..............................65

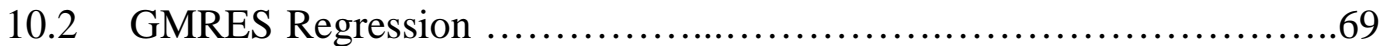

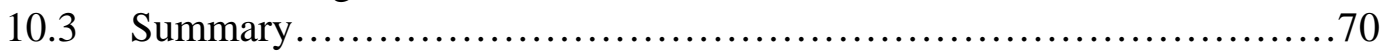

Chapter 11 Dimensionless Time and Optimum Coarse-Grid Size......................71

11.1 Dimensionless Time.............................................. 71

11.2 LaPlace SOR: Optimization of the Nested-Grid Method...................73

11.3 GMRES: Optimization of the Nested-Grid Method..........................74

11.4 Weber SOR: Optimization of the Nested-Grid Method...................75

11.5 Comparison of the Performance of the Different Methods................76

11.6 Optimal Number of Fixed Points......................................78

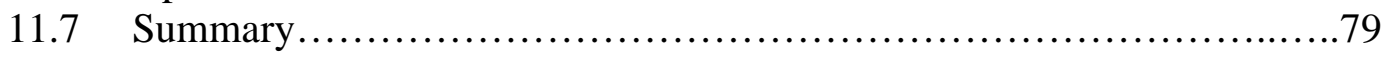

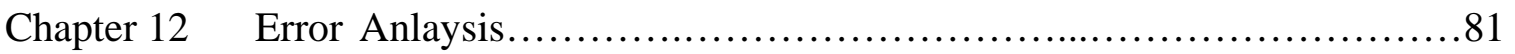

$12.1 \quad$ Error Analysis Results..............................................82 


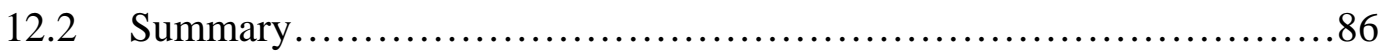

Chapter 13 Multiple Nested-Grid Analysis.................................................................

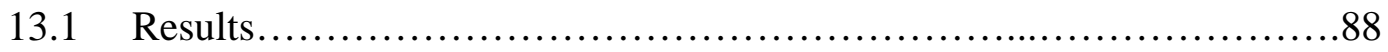

13.2 Practical Multiple Nested-Grid Study ................................90

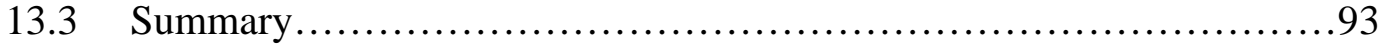

Chapter 14 Conclusion and Future Work..........................................95

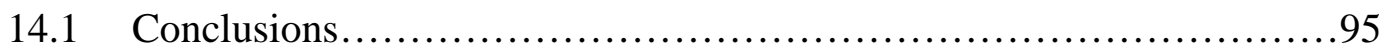

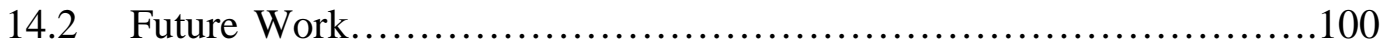

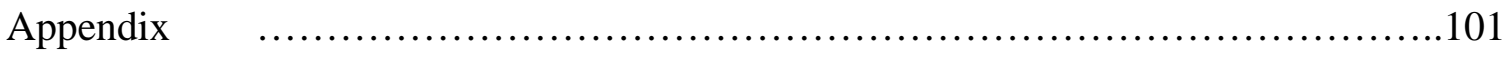

Appendix A Tables Referred to in Body of Thesis..................................103

Appendix B Original LaPlace and GMRES Regression and Dimensionless Time Analysis..........................................................111

Appendix C Original Weber Regression and Dimensionless Time Analysis..........123

Appendix D Optimal Over-Relaxation Factor Finder...............................129

Appendix E Summary of Computer Programs......................................139

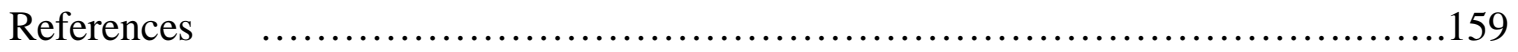




\section{LIST OF TABLES}

Table

$\underline{\text { Page }}$

I. General Objectives of Study..........................................13

II. $\quad$ Grid Sizes and Dimensions........................................

III. $\quad$ Fine-Grid Sizes Used in Nested-Grid Study..............................34

IV. $\quad$ Course-Grid Sizes............................................. 34

V. $\quad$ Grid Dimensions and Size............................................ 58

VI. $\quad$ Nested-Grid Points Take From a 5x5x10 Grid..........................84

VII. $\quad$ Nested-Grid Points Taken From a 15x15x30 Grid........................84

VIII. Nested-Grid Points Taken From a 5x5x10 Grid.............................84

IX. Nested-Grid Points Taken From a 15x15x30 Grid.........................84

X. $\quad$ Size of Coarse and Fine Grids in Nested-Grid Method.......................88

XI. Dimensionless Time Required to Solve Nested-Grid Method.................89

A.XII Nested-Grid Configurations...........................................103

A.XIII LaPlace SOR: Fine and Nested-Grid Results..........................103

A.XIV GMRES: Fine and Nested-Grid Results.............................104

A.XV. Interpolation Study ............................................ 105

A.XVI. Interpolation Study: Comparison with No Interpolation...................105

A.XVII. Weber SOR: Fine and Nested-Grid Results with Weber’s Coefficients.....106

A.XVIII. LaPlace SOR: Dimensionless Time Results of Nested-Grid Method........107 
A.XIX GMRES: Dimensionless Time Results of Nested-Grid Method.............107

A.XX. Weber SOR: Dimensionless Time Results of Nested-Grid Method..........108

A.XXI Absolute Difference from a $10^{-9}$ Solution...............................108

A.XXII Percent Difference from $10^{-9}$ Solution.....................................109

B.XXIII Laplace SOR: Fine and Nested-Grid Results............................111

B.XXIV Fine and Nested-Grid Results for GMRES...................................113

B.XXV Dimensionless Time Results of Nested-Grid Method.......................117

B.XXVI Dimensionless Time Results of Nested-Grid Method......................120

C.XXVII Weber SOR: Fine and Nested-Grid Results with Weber's Coefficients.....123

C.XXVIII Performance of Nested-Grid Method Using Weber's Coefficients............127 


\section{LIST OF FIGURES}

Figure

$\underline{\text { Page }}$

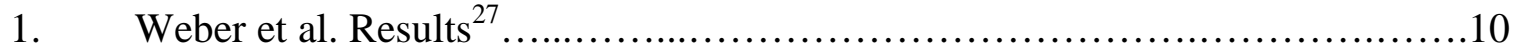

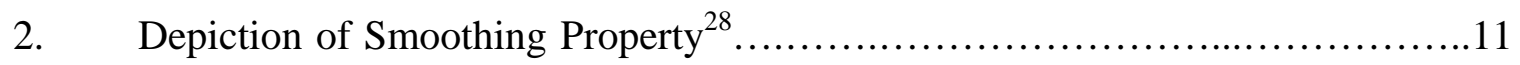

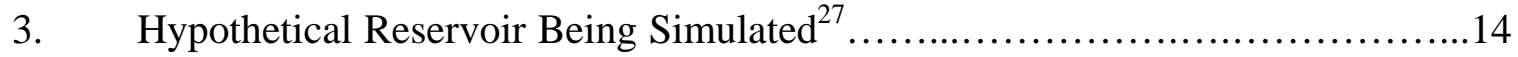

4. Determination of the Optimal Over-Relaxation Factor.........................21

5. Direct Methods: Time required for Convergence as a Function of Grid Size.....25

6. Direct Methods: Memory (RAM) Requirements as a Function of Grid Size......27

7. Iterative Methods: Time Required for Convergence as a Function of Grid

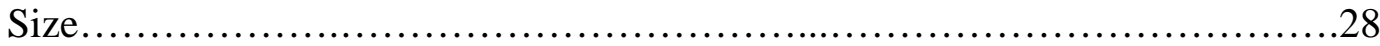

8. Iterative Methods: Memory (RAM) Requirements as a Function of Grid Size..................................................................

9. Top and Side Plane Views of Fixed Points in a 5x5x10 Grid....................35

10. Nested-Grid Configurations: Percentage of Fixed Points........................36

11. LaPlace SOR: Time Required for Convergence of Various Nested-Grids..........38

12. GMRES: Time Required for Convergence as a Function of Grid Size.............39

13. Comparison of Time Required to Obtain Full-Fine Grid Pressure Solution With and Without Interpolation..........................................45

14. Application of Weber's Coefficients.......................................50

15. Coarse Grid Dimensions in Feet..........................................59

16. 5x5x10 Coarse-Grid Pressures Nested Into a 15x15x30 Grid....................60 
17. Weber SOR: Time Required for Convergence of Various Nested-Grid Configurations.

18. LaPlace and Weber SOR Combined Data Set Regression.........................67

19. LaPlace and Weber SORCombined ORF Regression.............................69

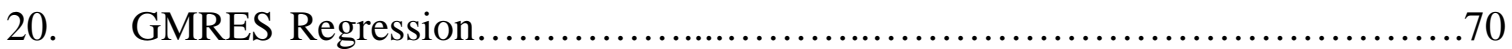

21. Time/Iteration as a Function of Total Grid Size ................................71

22. Laplace SOR: Improvement Obtained by the Nested-Grid Method.................73

23. GMRES: Improvement Obtained by the Nested-Grid Method.....................75

24. Weber SOR: Improvement Obtained by the Nested-Grid Method...................76

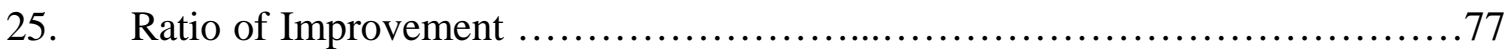

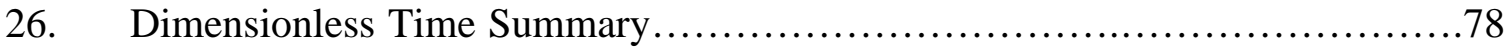

27. Optimal Number of Fixed Points..............................................79

28. Multiple Nested-Grid Analysis: $10^{3}, 10^{5}$, and $10^{6}$ Grid Sizes.....................90

29. Practical Nested-Grid Configuration.......................................91

30. A Look at Optimal, Practical and No Nested Grid Configurations..................92

B.31. LaPlace SOR Time/Iteration as a Function of Total Grid.......................115

B.32. Improvement Obtained by the Nested-Grid Method...........................116

B.33. Optimal Number of Coarse-Grid Points Nested Into Fine Grid..................118

B.34. GMRES: Time/Iteration as a Function of Total Grid Size........................119

B.35. Optimized Nested-Grid Method Using SOR Compared with GMRES............120

B.36. Comparison of Improvement Obtained by the Nested-Grid Method for

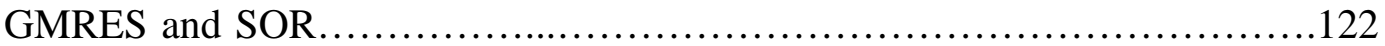

C.37. Weber Time/Iteration as a Function of Total Grid Size...........................125

C.38. Improvement Obtained by Nested-Grid Method...............................126 
C.39. Comparison of Dimensionless Time for all Solution Algorithms...............127

D.40. ORF 1.0

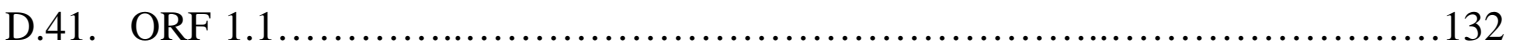

D.42. ORF 1.2

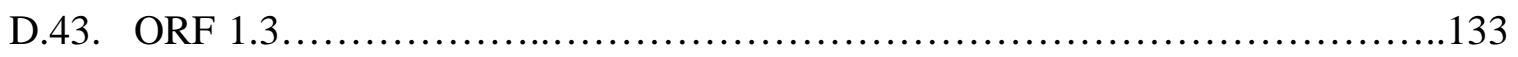

D.44. ORF1.4........................................................ 134

D.45. ORF1.5........................................................ 134

D.46. ORF1.6........................................................ 135

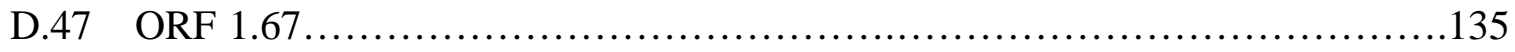

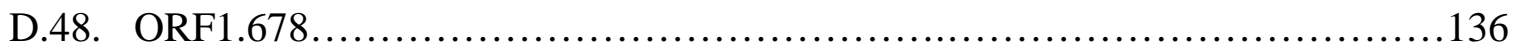

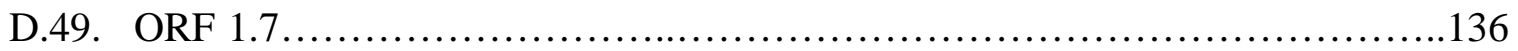

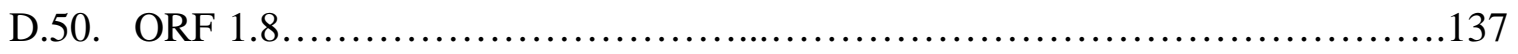

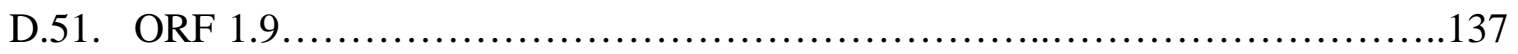

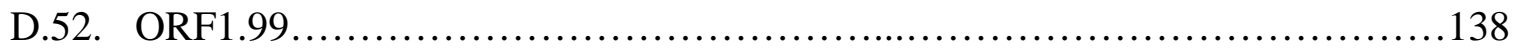




\section{NOMENCLATURE}

a

C

i,j,k

n

$\mathrm{P}$

q

r

$\mathrm{x}, \mathrm{y}, \mathrm{z}$

A, B, C, D

$\mathrm{Fx}, \mathrm{Fy}, \mathrm{Fz}$

K

$\mathrm{K}^{\prime}$

$\mathrm{N}$

$\mathrm{P}$

$\mathrm{N}_{\text {ITER }}$

$\mathrm{N}_{\mathrm{FG}}$

$\mathrm{N}_{\text {CG }}$

$\mathrm{N}_{\text {LAPLACE }}$

$\mathrm{N}_{\text {WEBER }}$

$\mathrm{N}_{\mathrm{CG}}{ }^{\circ}$

$\mathrm{N}_{\mathrm{FG}}{ }^{`}$

$\mathrm{Q}_{\mathrm{n}}$

$r_{n}$

$t_{d}$

$t_{\text {total }}$

$\mathrm{t}_{\text {coarse-grid }}$

$t_{\text {nested-grid }}$

$\lambda$

$\mu$

$\omega$

$\omega_{\text {opt }}$

$\Omega$

$+$

Proportionality constant

Integration constant

Grid point location in $\mathrm{x}, \mathrm{y}, \mathrm{z}$ respectively

Number of grid refinements; number of wells

Pressure

Flux of fluid through porous media

Distance to the wells

Cartesian coordinates

Variables determined by regression

Fraction of $\mathrm{x}, \mathrm{y}$, and $\mathrm{z}$-distances that the fine-grid points are from point (1)

of the course-grid block

Permeability

Pseudo-permeability

Parameter used to describe effectiveness of well, determined by regression

Pressure

Number of Iterations

Number of fine-grid points

Number of coarse-grid points

Parameter used to describe effectiveness of well for LaPlace SOR

Parameter used to describe effectiveness of well for Weber SOR

$\mathrm{N}_{\mathrm{CG}}-\mathrm{N}$

$\mathrm{N}_{\mathrm{FG}}-\mathrm{N}$

Volumetric injection rate of well $n$

Distance to well $\mathrm{n}$

Dimensionless time

Total dimensionless time

Dimensionless time required to calculate coarse-grid solution

Dimensionless time required to calculate a given nested-grid solution

Ratio of pseudo-permeability to actual permeability

Fluid viscosity

Over-relaxation factor

Optimal Over-relaxation factor

Solid angle

Positive cell face

Negative cell face 


\section{CHAPTER 1}

\section{INTRODUCTION}

This thesis proposes a new method of linear algebra that provides a rapid solution of very large systems of equations. This work was motivated by the need for such a technology in reservoir simulation. However, the method is likely to be valuable in other disciplines where large systems of linear equations are encountered, such as in computational fluid mechanics.

Oil is the lifeblood of America's economy. It presently supplies more than $40 \%$ of the nation's total energy demands. ${ }^{1}$ Recently the Department of Energy (DOE) has expressed two key concerns over oil in America: (1) maintaining an immediate readiness to respond to oil supply disruptions and (2) keeping America's oil fields producing in the future. The DOE has determined that one way to prevent an oil supply disruption is to make certain that domestic production of oil is maintained. Remaining U.S. oil fields are becoming progressively more costly to produce because much of the easily produced oil has already been recovered. Better technology is needed to locate and produce the

residual oil. ${ }^{1}$ Many of the emerging technologies that will keep U.S. oil fields producing long into the future do and will employ reservoir simulators. 


\subsection{Technologies That Depend on Reservoir Simulation}

Reservoir simulation became feasible some thirty years ago with the dawn of the computer age. Since that time, computer technology has increased greatly. Computer hardware and software have become continuously more powerful at an astonishing rate. However, throughout the history of computers reservoir simulators have always taxed the very fastest machines, and despite these enormous advances in computer technology, reservoir simulators continue to strain the largest and fastest computer systems. The need for faster reservoir simulation remains as critical today as it has ever been. Emerging technologies in the oil industry that depend on reservoir simulators would be greatly enhanced by more accurate and faster reservoir simulations. Brief descriptions of some of the emerging technologies key to optimal oil recovery are described as follows.

Geostatistics: Geostatistics involves gathering large quantities of data from exposed rock out-crops, where measurements of physical properties can be made with relative ease. The statistical variations in physical properties observed in the out-crops are applied to data obtained from well cores, logs, and from surface seismic data for subterranean reservoirs. Many possible subsurface models of the same field, each with some probability of being correct, can then be generated. Simulation of all the geostatistical reservoir models is practical only when simulations can be made rapidly. Results of these reservoir simulations are valuable as they can provide a measure of a field's potential profitability as well as the associated risks. ${ }^{2}$

Automated History Matching: History matching plays a critical role in monitoring displacement processes, constructing good reservoir models, and predicting future reservoir performance. Production data are the most common type of reservoir data. Matching these data allows reservoir engineers to better characterize reservoir properties such as permeability and porosity. ${ }^{2}$ This process has been automated, and is relatively simple when the number of data values to be adjusted is small and when simulations can be accomplished rapidly. For more extensive applications, history matching remains a difficult and time-consuming task, as many simulations of the same field must be made to determine a data set that matches the production history of the field. ${ }^{2}$

Optimization: Optimization of reservoir production involves repeated simulations of a particular field to determine optimal reservoir design variables 
and design functions such as well location, geometry, completion intervals, well rates, well remedial treatments, etc. Optimization software is available, yet fast, accurate and robust reservoir models are essential to enhance the optimization process. $^{2}$

Smart Wells: Smart wells are equipped with down-hole measurement equipment, packers, and control valves. They use real-time simulations to control the production rate from different well segments through the use of packers, which isolate the various production intervals in a well, and valves, that control the amount of flow from each interval. Smart wells have the potential to significantly increase production (up to 65\%). ${ }^{3}$ Optimal real-time control theory, used to determine proper packer and valve settings, requires rapid reservoir simulation. $^{2}$

\subsection{Reservoir Descriptions}

Modern reservoir imaging techniques and advances in geological modeling are allowing very detailed reservoir descriptions. Often ten million cells or more may be used to describe reservoir rock properties. With current computers, most oil companies cannot afford to run routine fluid-flow simulations with more than about 100,000 grid blocks. ${ }^{4}$ Typical reservoir models generally contain $10,000-100,000$ cells. $^{5}$

The alternative to running multi-million cell simulation models that incorporate the geologic models, is "Upscaling". "Upscaling" is the practice by which a fine geologic model containing a detailed description of reservoir rock properties is replaced by a coarser scale description of equivalent reservoir rock properties which is more suitable for reservoir simulation. The coarsening of the geologic model can lead to reservoir simulation runs that can be completed in hours, as opposed to days. The challenge in applying this process is to retain an accurate representation of the physical characteristics of the geologic model critical to fluid flow. ${ }^{6}$ A variety of different methods has been proposed to upscale single and multiphase flow properties. Many

studies $^{7,8,9,10,11,12}$ and reviews ${ }^{4,5,13,14,15,16}$ have been conducted, yet all upscaling methods 
suffer from the problem that either, they make assumptions about the large-scale boundary conditions, which may significantly affect the results, or they require a fine-

grid solution to derive coarse grid properties. ${ }^{17}$ Other unsettled issues include grouping of upscaled relative permeabilities, robustness and process independence. There are still unresolved issues dealing with extending multi-phase scales up to three-phase flow, compositional flow, and flow in naturally occurring systems. ${ }^{5}$ Consequently, despite a huge literature on multiphase upscaling, the best approach is still very much an open issue. In fact, the current industry practice is to limit upscaling to single-phase properties only. ${ }^{17}$

In a recent review, M. A. Christie stated that, "The most promising methods of the last few years may be those that regard upscaling as an integral part of the solution of the flow equations, rather than as an external process which have the correct boundary conditions to provide the correct answer."5

\subsection{Reservoir Simulation Approaches}

In the May $17^{\text {th }}$, 2004, Oil \& Gas Journal, ${ }^{6}$ Scott Evans summarized four basic approaches that can be used for reservoir simulation in today's high performance computing environments.

"Four basic approaches that can be successfully used in reservoir simulation are available for subsurface modeling:

1. Traditional. A geologic model "upscaled" to a second model and used for reservoir simulation. The result is two models, only one of which is used for simulation.

2. Geologic. A geologic model run without coarsening in the reservoir simulator. The result is one model but with potentially very slow simulation runs 
3. Hybrid. One model generated, but with varying scale, in which the model has more detail where needed and less where it is not. The result is one model with potentially more acceptable simulation runs.

4. Multiscale. Two or more models, one fine and one coarse, linked and used simultaneously in the reservoir simulator. This is an area of industry research and would have each of the models used in the simulation as appropriate.”

The multiscale approach is a current area of research and development. This methodology will hopefully provide a way to more accurately represent the static and dynamic properties of a reservoir with minimal computational power. Recent studies have shown how to use both coarse and fine grid information during reservoir simulations.

\subsection{Review of Multiscale Methods}

In 1991, Ramé and Killough ${ }^{18}$ presented the first implementation of a multiscale simulation technique. The method used the implicit-pressure, explicit saturation (IMPES) procedure to decouple the pressure equation from the conservation equations numerically. A fourth-order finite element method was used to solve the pressure equation on each coarse grid. Fine-scale information was interpolated from the coarse grid using a splines-under-tension technique, and the conservation equations for fluid transport were solved on the fine grid. Time stepping was performed on the fine grid and after several timesteps the current mobilities on the fine grid were passed to the coarse grid to update the pressure field. 2D examples for miscible flow were presented.

In 1995 Guérillot and Verdière ${ }^{19}$ proposed a dual mesh method where the pressure field was first computed on a coarse grid, but where the saturation was moved on the fine grid. Using approximate boundary conditions, the velocity field was estimated within 
each coarse block by solving for the pressure. This technique was applied to a 2D singlephase model and in 1996 they extended their work to multiphase flow. ${ }^{20}$ They showed the results in 2D for two-phase flow models with simplified injection-production boundary conditions. Comparison of the time of calculation spent for the full fine-grid calculation and the dual mesh method gave a speed-up factor from 5 to 7 .

In $1996 \mathrm{Hou}$ and $\mathrm{Wu}^{21}$ presented the derivation of a mathematically rigorous multiscale finite-element method for solving the class of elliptical problems that arise from composite material (steady-heat conduction through a composite material with tubular fiber reinforcement in a matrix) and flows in porous media. By constructing multiscale finite-element base functions that were adaptive to the local property of the differential operator, this method was able to capture efficiently the large-scale behavior of the solution without resolving the small-scale features. Results for flow in porous media were provided in 2D without gravity and capillary effects.

In 1999 Guedes and Schoiozer ${ }^{22}$ implemented the same methodology as Guérillot and Verdière, using an upscaling method developed by Hermitte and Guerillot. ${ }^{23}$ They provided results in 2D and included gravity effects. Well boundary conditions were considered as sources or sinks applied in one grid block.

Also in 1999, Gautier et al. ${ }^{24}$ presented a similar approach using streamline-based simulation (an IMPES method). The pressure solve method (psm) was used to upscale the transmissivities for each coarse-grid block from petrophysical properties defined on a fine grid. Gravity effects and wells where included. The well pressures were determined using Peaceman's well model, and special attention was given to keep equivalent fluxes on both coarse and fine scales. The method was tested on a series of waterflood problems 
and it was demonstrated that the method could give accurate estimates of oil production for large 3D models up to 8.5 times faster than direct simulation using streamlines on the fine grid. The results were very efficient in terms of CPU time and memory management. Arbogast and Bryant ${ }^{25}$ introduced a slightly different dual grid approach in 2001 by using Green function methods to upscale transmissivities and a mixed finite-element method to solve the pressure field. Gravity and capillary effects were considered in their methodology. Similar to Guatier et al. ${ }^{24}$, they observed a reduction ratio for the time of calculation of about 2 to 10 compared to fine-grid simulation.

Audigane and Blunt (2004) ${ }^{17}$ present an extension of the dual-mesh method of Guérillot and Verdière to 3D cases and included gravity and wells. Using an IMPES method, the pressure field was solved on the coarse mesh with a conventional finitedifference scheme. Transmissivities were upscaled either with the psm method or with a simple geometric average (ga). The pressure field was reconstructed on the fine mesh using flux boundary conditions from the coarse-grid simulation.

Multiscale methods continue to develop and make advances in reducing the cpu time of reservoir simulations. The solution of the pressure equation is the most timeconsuming step in any reservoir simulation ${ }^{5}$, and it has become more evident that improved mathematics, which will allow faster and more accurate simulations, is fundamental to improved reservoir simulation capabilities.

In reservoir simulation, the primary concern is movement of gas, oil, and water in the reservoir. ${ }^{26}$ These fluids flow as a result of pressure variations in the reservoir. Hence, the accurate prediction of reservoir pressures is essential to a good reservoir simulator. This work proposes a new linear algebra technique for the solving of finely-gridded 
reservoir pressures. The new method is multiscale, in that it involves the calculation of the reservoir pressures on a coarse grid and then uses the coarse-grid solution in a nested grid to calculate fine-grid pressures. Improved mathematics are key to the method and permit faster and more accurate solutions of the pressure equation.

\subsection{Summary}

Better technology is needed to produce oil and gas reserves in a more effective manner. Many developing technologies that improve oil and gas production would be greatly enhanced by faster and more accurate reservoir simulators. In approaching reservoir simulation, the multiscale method is an area of industry research which has been shown by various researchers to reduce the computational time required to complete reservoir simulations. Calculation of reservoir pressures is the most time consuming step in any reservoir simulator; this thesis proposes a new linear algebraic method that incorporates the multiscale approach to calculate reservoir simulation pressures in an accurate and fast manner. 


\section{CHAPTER 2}

\section{BACKGROUND}

\subsection{Weber's Equations and Nested-Grid Description}

The new pressure-solution method developed in this thesis is based on the work of Weber et al. ${ }^{27}$ They proposed that finite-difference equations, used to represent the pressure equation, be based on mathematical expressions that incorporate the physics of the process instead of on traditional polynomial expressions. In modeling reservoir pressures, equations incorporating the physically realistic $\ln (\mathrm{r})$ dependence on pressure for reservoirs with straight line wells, and a 1/r dependence for reservoirs with more complex well geometries were used ( $\mathrm{r}$ is the distance to the wells). Weber investigated formulations in which $\ln (\mathrm{r})$ 's and 1/r's were summed over all the wells in the reservoir, and in which only the single, closest well value was used. The results of Weber's study that incorporated a $1 / \mathrm{r}$ dependence into the finite-difference equations are shown in Figure 1 . The figure compares the accuracy of the pressures calculated by various methods for an 11x11x22 grid of a rectangular reservoir of similar geometry to that used in this study. The different methods were compared with an analytical solution generated by Weber.

The new finite-difference equations showed a four-order-of-magnitude improvement in accuracy compared with traditional polynomial based finite-difference equations and approximately three-orders-of-magnitude improvement over Peaceman's 
Correction. This dramatic improvement in accuracy was the catalyst for the development of a new method to calculate finely-gridded pressures.

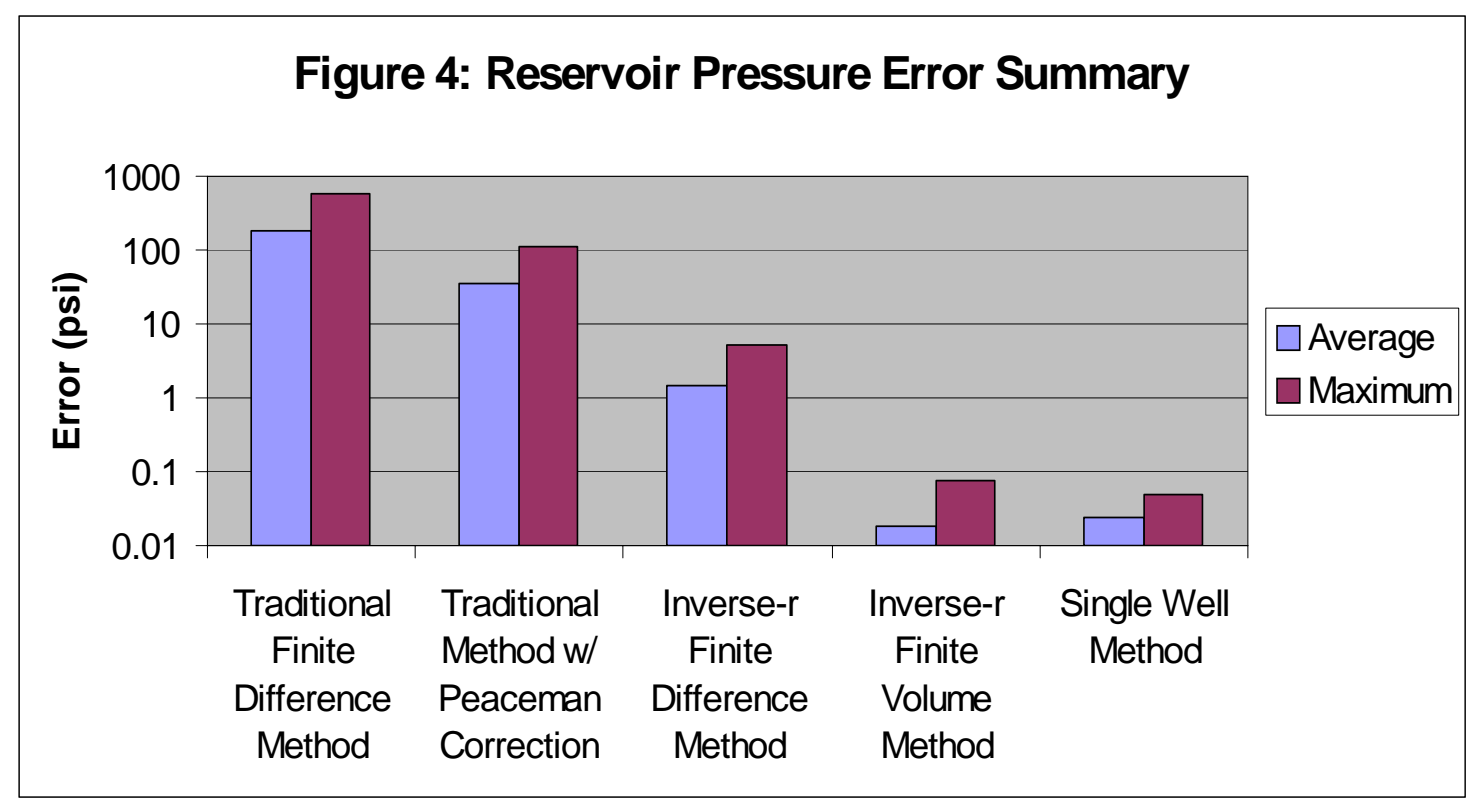

Figure 1. Weber et al. Results ${ }^{27}$

The new method involves two steps: (1) The creation of a course-grid solution using Weber's finite-difference equations that incorporate the physics of the flow to obtain an accurate pressure solution on a small, coarse grid, and (2) nesting the coarsegrid solutions into a desired fine grid and solving the system of equations to obtain detailed pressures that honor the nested-course-grid pressures. The improved mathematics of Weber et al. is vital to this method, as it permits the generation of accurate solutions on coarse grids. 


\subsection{Comparison to Multigrid Method}

This method is not a multigrid method, yet it does utilize some of the basic ideas of the multigrid method, namely coarse-grid relaxation and nested iteration. Multigrid methods are built on the fact that many standard iterative methods possess a smoothing property. The smoothing property describes the fact that as iterative methods progress to convergence the reduction in error decreases and becomes smooth. Figure 2 is taken from the book “A Multigrid Tutorial” by William L. Briggs ${ }^{28}$ and shows this smoothing effect for the weighted Jacobi method in a plot of the absolute error versus iteration number. The error decreases quickly within the first five iterations and then decreases slowly.

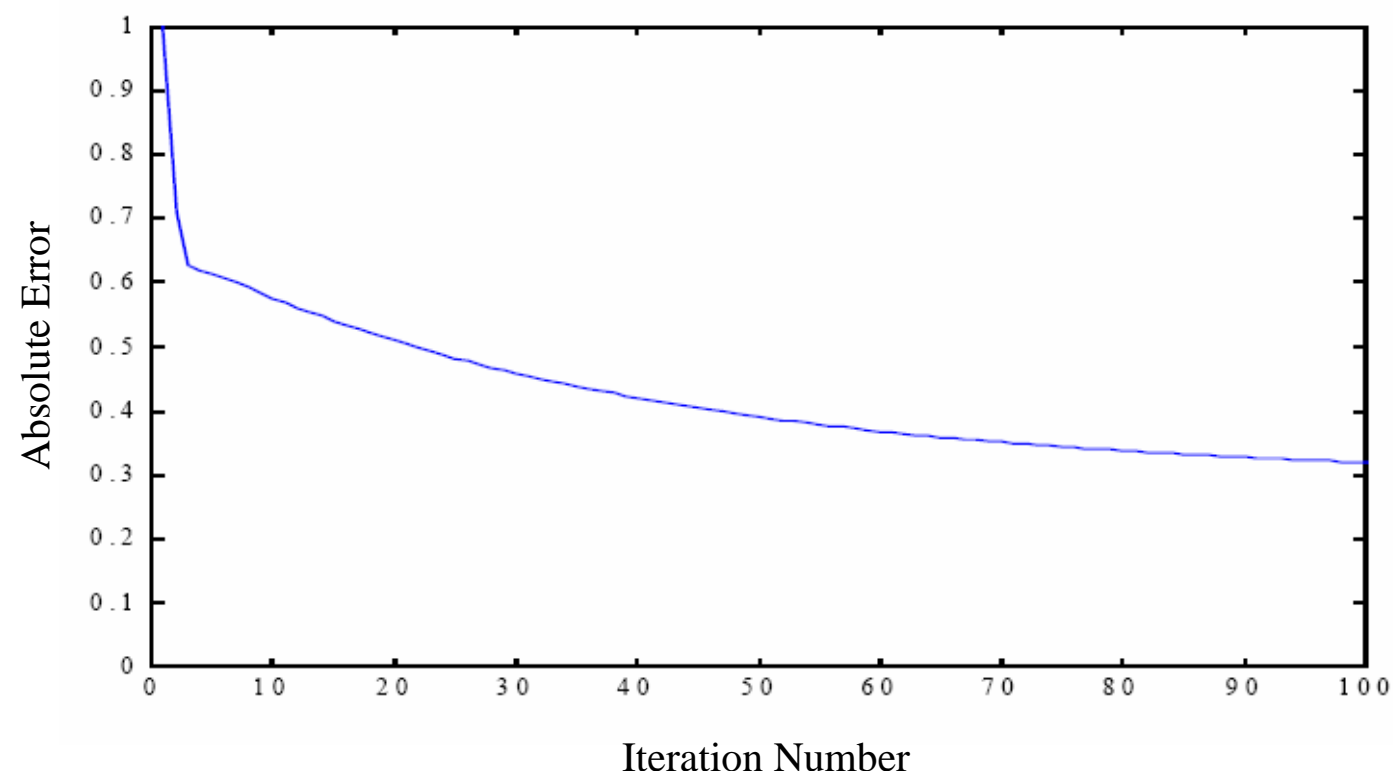

Figure 2. Depiction of Smoothing Property ${ }^{28}$

The initial rapid decrease in error is associated with the quick elimination of highfrequency modes, and the slow decrease is due to the presence of low-frequency modes. The modes are Fourier modes where a small wave number, $\mathrm{k}$, is associated with long 
smooth waves, and large values of k correspond to highly oscillatory waves. As stated earlier, many standard iterative methods possess this smoothing property which makes them very effective at eliminating high-frequency or oscillatory components of the error, while leaving the low-frequency or smooth components relatively unchanged. Through the use of coarse grids, the multigrid method puts the smoothing property to good use as the smooth error is relatively more oscillatory on coarser grids. Hence relaxation becomes more effective on coarser grids. Coarse grids can be used to compute an improved initial guess for fine-grid relaxation, and a reliable way to improve the relaxation scheme on the fine grid is to use a good initial guess. This well-known technique of using a coarse grid to generate improved initial guesses on a fine grid is called nested iteration. In a nut shell, multigrid methods relax on a fine grid until the smooth error is reached and the high frequency oscillations have been eliminated. The grid is then restricted to a coarse grid where the error is relatively more oscillatory. The grid is again relaxed until smooth error is reached. The coarse grid points become a new and improved initial guess for the fine grid where the coarse grid points are mapped to the fine grid through interpolation. The fine grid is relaxed again and the process continued to convergence. ${ }^{28}$

The research of this thesis investigates the potential value of implementing, in a nested-grid/multiscale manner, the new finite-difference equations developed by Weber et al. However, unlike the multigrid method described previously, the new method can potentially be completed in only two steps, one using a course grid and a second using a fine grid. Only two grids are necessary because of the very high accuracy of the coarsegrid solution using Weber’s solution. 
This thesis describes development of this new method. The steps involved are outlined in Table I.

Table I. General Objectives of Study

\section{Objectives of Study}

Test performance of various solvers as a function of grid size (Chapters 3,4)

Analyze results to determine the best solver(s) for coarse and fine grids. Selected solver(s) will be used in nested-grid solution method (Chapter 5)

Implement best solver(s) in the nested-grid solution method (Chapter 6)

Consider potential value of applying interpolated values to initial solution guesses (Chapter 7)

Apply Weber’s coefficients in nested-grid solution method (Chapters 8,9)

Regress all results to determine potential correlations that fit data (Chapter 10)

Conduct a dimensionless time analysis using correlations determined from regressions and optimize the nested-grid configuration (Chapter 11)

Analyze the error of the nested-grid solution method (Chapter 12)

Investigate the use of multiple nested-grids to improve method further (Chapter 13)

\subsection{Reservoir Description}

In this work, the reservoir geometry considered is shown in Figure 3. Injection at 1,500 psi occurs in one well; production at $-1,500$ psi occurs in the other well. The two wells are centered, one in each of the two cubic elements comprising the threedimensional rectangular reservoir. The boundary conditions of the reservoir are no flow, i.e. the pressure gradients in the direction normal to the boundaries are zero. 


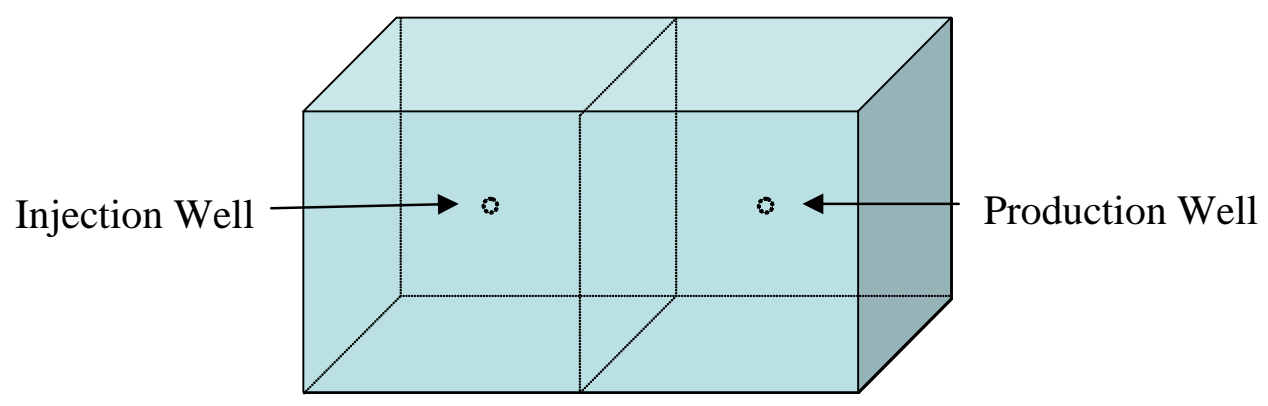

Figure 3. Hypothetical Reservoir Being Simulated ${ }^{27}$

The dimensions of the reservoir are in the following ratio: 1x1x2. The actual reservoir dimensions were factored in by scaling the well radius with the size of the grid block. For grid blocks of 100 foot dimensions, the well radius of three inches becomes 0.0025. Although gravity effects are assumed negligible, the reservoir was considered to lie with its largest dimension in the horizontal plane.

\subsection{Solution of the Pressure Equation}

The pressure equation is written in terms of average pressure for the conservation of mass flowing through porous material. The incompressible, steady-state, threedimensional pressure in a reservoir for which the mobility is everywhere uniform, is given by the Laplace equation:

$$
\frac{\partial^{2} P}{\partial x^{2}}+\frac{\partial^{2} P}{\partial y^{2}}+\frac{\partial^{2} P}{\partial z^{2}}=0
$$


Replacing each of the second derivatives by second-order, centered-difference approximations at grid point $(\mathrm{i}, \mathrm{j}, \mathrm{k})$ yields

$$
\frac{P_{i+1, j, k}-2 P_{i, j, k}+P_{i-1, j, k}}{\Delta x^{2}}+\frac{P_{i, j+1, k}-2 P_{i, j, k}+P_{i, j-1, k}}{\Delta y^{2}}+\frac{P_{i, j, k+1}-2 P_{i, j, k}+P_{i, j, k-1}}{\Delta z^{2}}=0
$$

In the special case where $\Delta \mathrm{x}=\Delta \mathrm{y}=\Delta \mathrm{z}$ the grid aspect ratio is unity and Equation (2-2) becomes

$$
P_{i, j, k}=\frac{P_{i+1, j, k}+P_{i-1, j, k}+P_{i, j+1, k}+P_{i, j-1, k}+P_{i, j, k+1}+P_{i, j, k-1}}{6}
$$

This is the traditional, finite-difference formulation used to solve the pressure equation in three dimensions.

\subsection{Summary}

Weber et. $\mathrm{al}^{27}$ developed finite-difference equations that incorporate physics of flow and allow for pressure solutions, four-orders-of-magnitude more accurate, to be generated on coarse grids. The proposed new calculation method developed in this thesis takes advantage of Weber's accurate coarse-grid solutions in a nested-grid calculation method to determine finely-gridded reservoir simulation pressures. Although this method incorporates some of the basic ideas of multigrid methods, namely coarse-grid relaxation and nested iteration, it is not a multigrid method as the coarse-grid pressure solutions are nested and fixed throughout the entire relaxation process of the fine grid. The reservoir 
being simulated is of an idealized nature. The simplify assumptions include: (1)

homogeneous permeability, (2) neglected gravity effects, (3) incompressibility (4)

steady-state, and (5) dimensions of reservoir and placement of wells generate a symmetric pressure solution. 


\section{CHAPTER 3}

\section{TESTING THE PERFORMANCE OF VARIOUS SOLVERS AS A FUNCTION OF GRID SIZE}

Reservoir simulations can be made at varying degrees of grid refinement ranging, as mentioned previously, from hundreds to millions of grid blocks. For this study, it was important to know how various solvers performed as a function of grid size so that the best solver(s) could be used for all grid sizes in the nested-grid method.

The study was initialized by considering the performance of various numerical methods for the solution of systems of linear algebraic equations as a function of grid size on a standard desktop computer. The computer used four Intel (R) Pentium (R) processors running at $1.80 \mathrm{GHz}$, and $655 \mathrm{MB}$ of RAM. In the entire study, swapping RAM data to the hard drive paging was not apparent. Hence the same performance increase would be expected on any computer (workstation, supercomputer) with sufficient memory to avoid paging. Both direct and iterative methods were considered and compared. The performance metrics included convergence time, iterations required to converge, and run-time memory requirements.

To study the performance of the various linear algebraic solvers as a function of grid size, a range of test grid sizes was selected. The grids were constructed so that the wells were always in the center of their respective cells. Table II summarizes the various grid sizes used. 
Table II. Grid Sizes and Dimensions

\begin{tabular}{|c|c|}
\hline Grid Size & Grid Dimensions \\
\hline 250 & $5 \times 5 \times 10$ \\
\hline 686 & $7 \times 7 \times 14$ \\
\hline 1458 & $9 \times 9 \times 18$ \\
\hline 2662 & $11 \times 11 \times 22$ \\
\hline 9826 & $17 \times 17 \times 34$ \\
\hline 18522 & $21 \times 21 \times 42$ \\
\hline 71874 & $33 \times 33 \times 66$ \\
\hline 101306 & $37 \times 37 \times 74$ \\
\hline 265302 & $51 \times 51 \times 102$ \\
\hline 549250 & $65 \times 65 \times 130$ \\
\hline
\end{tabular}

\subsection{Direct Methods}

Direct solution methods perform very well for small grids, but can require excessive computational effort and computer memory as the number of grid points increases. ${ }^{29}$ Direct methods might be preferable for the course-grid solution, while iterative methods might be required for the fine grid. The direct elimination methods analyzed were Gauss Elimination ${ }^{29}$, the Doolittle LU factorization method ${ }^{29}$, and a band solver, DGBSV, from the LAPACK library. ${ }^{30}$ The programs were developed using Compaq Visual Fortran 6.5 (Fortran 90). Default compiler options were used throughout so that others could duplicate results.

\subsection{Iterative Methods}

Iterative methods can be divided into two general categories, stationary and nonstationary. ${ }^{31}$ Three standard stationary iterative methods were considered, namely Jacobi, Gauss-Seidel, and Successive-Over-Relaxation (SOR). Five advanced nonstationary iterative methods from MATLAB $7.0^{32}$ and a hybrid of direct and iterative 
methods, Line Successive-Over-Relaxation (LSOR), were also considered. Iterative methods should be better suited for the large systems of equations required in this study. The computer programs for these solvers, other than the five found in MATLAB, were developed in-house with the incorporation of the Thomas algorithm (DGTSV) from the LAPACK library ${ }^{30}$ for LSOR. The in-house programs were developed in Compaq Visual Fortran 6.5 (Fortran 90).

Some iterative methods require diagonal dominance to guarantee convergence and in general are less robust than direct solvers. The system of equations arising from the seven-point, second-order approximation of the Laplace equation used in the simulations of this study is always diagonally dominant. Hence, such iterative solvers work well.

For any iterative method, an initial approximation must be made for the solution to start the process. Several choices are available: (1) Simply let the solution (in this case the value of the pressure $P_{i, j, k}$ at the various grid points) equal zero at all non-specified points; (2) Approximate $\mathrm{P}_{\mathrm{i}, \mathrm{j}, \mathrm{k}}$ by some average of the well pressures and or boundary conditions; or (3) Construct a solution on a course grid, and map the course-grid values onto the fine grid through interpolation. ${ }^{29}$ For the determination of the best solver to be used in the nested-grid method, the initial $\mathrm{P}_{\mathrm{i}, \mathrm{j}, \mathrm{k}}$ solution vector was set to 0.0 everywhere except at the wells, which was set to the average of the well pressures, 1500 and -1500 . However, later in the study (See Chapters 6 and 9), coarse-grid points would be nested into the fine grid, and three-dimensional linear interpolation would also be considered to establish good starting values for iterative methods on the fine grid. 
Iterative methods only generate an approximate solution after a finite number of iterative steps; the iterative process terminates when it meets a specified convergence criterion. In general, the number of iterations required to satisfy the convergence criterion is influenced by diagonal dominance, method of iteration, initial solution vector, and the convergence criterion itself. ${ }^{29}$ The convergence criterion used by the in-house iterative methods in this study is described by the following equation.

$$
\left|\mathrm{P}_{1,1,1}+\mathrm{P}_{\text {Imax }, \text { Imax }, K \max }\right| \leq 10^{-6}
$$

$\mathrm{P}_{1,1,1}$ and $\mathrm{P}_{\mathrm{Imax}, \mathrm{Imax}, \mathrm{Kmax}}$ are the values of the pressures at opposite corner points of the grid furthest from each other in three dimensions. The symmetry of the problem indicates that $\mathrm{P}_{1,1,1}$ equals $\mathrm{P}_{\text {Imax }, \text { Imax,Kmax }}$ in a properly converged solution. $\mathrm{P}_{1,1,1}$ is the first to be calculated and hence is the least converged. $\mathrm{P}_{\text {Imax,Ima,Kmax }}$ is the last to be calculated and would be expected to be the most converged. Since the pressures are initially at zero and relax to their solution values asymptotically, with points near the wells changing most rapidly, this convergence criteria should represent the maximum error in the solution after many iterations.

For the iterative method of SOR and the hybrid method of LSOR, another key factor in the determination of the number of iterations required for convergence was the value of the over-relaxation factor, $\omega$. When $\omega$ equals one, SOR yields the Gauss-Seidel method. When $\omega$ is greater than one, but less than two, the system is over-relaxed; when the $\omega$ factor is equal two or greater than two, the system becomes unstable. Figure 4 shows a plot of the iterations required for convergence as a function of the over- 
relaxation factor for a $17 \times 17 \times 34$ grid solved by SOR. In this case it is apparent that by using the optimal over-relaxation factor one reduces the number of iterations required for convergence by approximately two orders of magnitude in comparison with the GaussSeidel iteration method.

Optimal Over-Relaxation Factor: 17x17x34

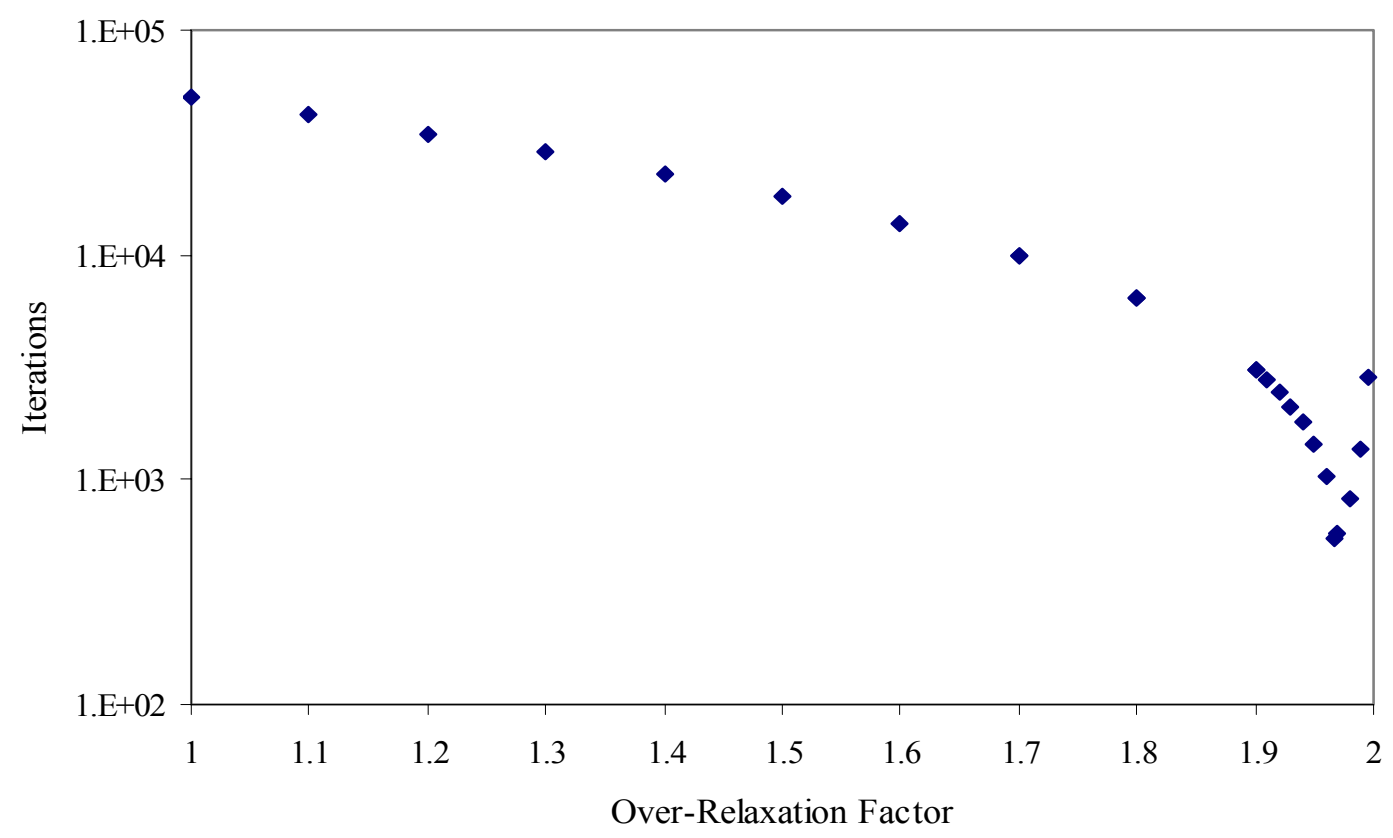

Figure 4. Determination of the Optimal Over-Relaxation Factor

The relaxation factor does not change the final solution since it multiplies the residual, which is zero when the final solution is reached. The major difficulty with the overrelaxation method is the determination of the best value for $\omega$. In general, the optimal value of the over-relaxation factor, $\omega_{\mathrm{opt}}$, depends on the size of the system of equations and the nature of the equations. As a general rule, larger values of $\omega_{\mathrm{opt}}$ are associated with larger systems of equations. ${ }^{29}$ The optimum value of $\omega$ was determined by 
experimentation for the various grid sizes considered in this study. The process of finding $\omega_{\mathrm{opt}}$ was straight forward but often time consuming, especially for large grids.

\subsection{MATLAB 7.0 Iterative Methods}

MATLAB is a high-performance language for technical computing; the name stands for matrix laboratory. MATLAB incorporates LAPACK and BLAS libraries in its software for matrix computation. Nine functions are available in MATLAB that implement advanced iterative methods for sparse systems of simultaneous linear systems. Of these nine, five where considered: Biconjugate Gradient (BICG), Biconjugate Gradient stabilized (BICGSTAB), LSQR implementation of Conjugate Gradients on the Normal Equations (LSQR), Generalized Minimum Residual (GMRES), and Quasiminimal Residual (QMR). GMRES is a Krylove Subspace Method and was recommended for use in this study by simulation developers from ConocoPhillips. All of the MATAB algorithms were implemented without preconditioners, and the magnitude of the convergence tolerance was $10^{-6}$.

\subsection{Summary}

A standard desktop computer was used for the study of the performance of various solvers as a function of gird size. Direct solution methods considered were Gauss Elimination $^{29}$, the Doolittle LU factorization method $^{29}$, and a band solver, DGBSV, from

the LAPACK library. ${ }^{30}$ Stationary iterative methods considered for the study were Jacobi, Gauss-Seidel, and Successive-Over-Relaxation (SOR). Five nonstationary iterative methods from MATLAB 7.0: Biconjugate Gradient (BICG), Biconjugate Gradient 
stabilized (BICGSTAB), LSQR implementation of Conjugate Gradients on the Normal Equations (LSQR), Generalized Minimum Residual (GMRES), and Quasiminimal Residual (QMR) were considered. A hybrid of direct and iterative methods, Line Successive-Over-Relaxation (LSOR), was also considered. SOR and LSOR require the determination of an optimal over-relaxation factor. 


\section{CHAPTER 4}

\section{PERFORMANCE OF VARIOUS SOLVERS}

\subsection{Direct Methods: Results}

Figure 5 shows a plot of convergence time as a function of grid size for the direct methods of Gauss Elimination (GE), Doolittle LU factorization (LU) and the band solver, DGBSV, from the LAPACK library. Due to the computational limits of the computer, the solvers’ performance was only considered on smaller grid sizes.

Direct Methods: Time vs Grid Size

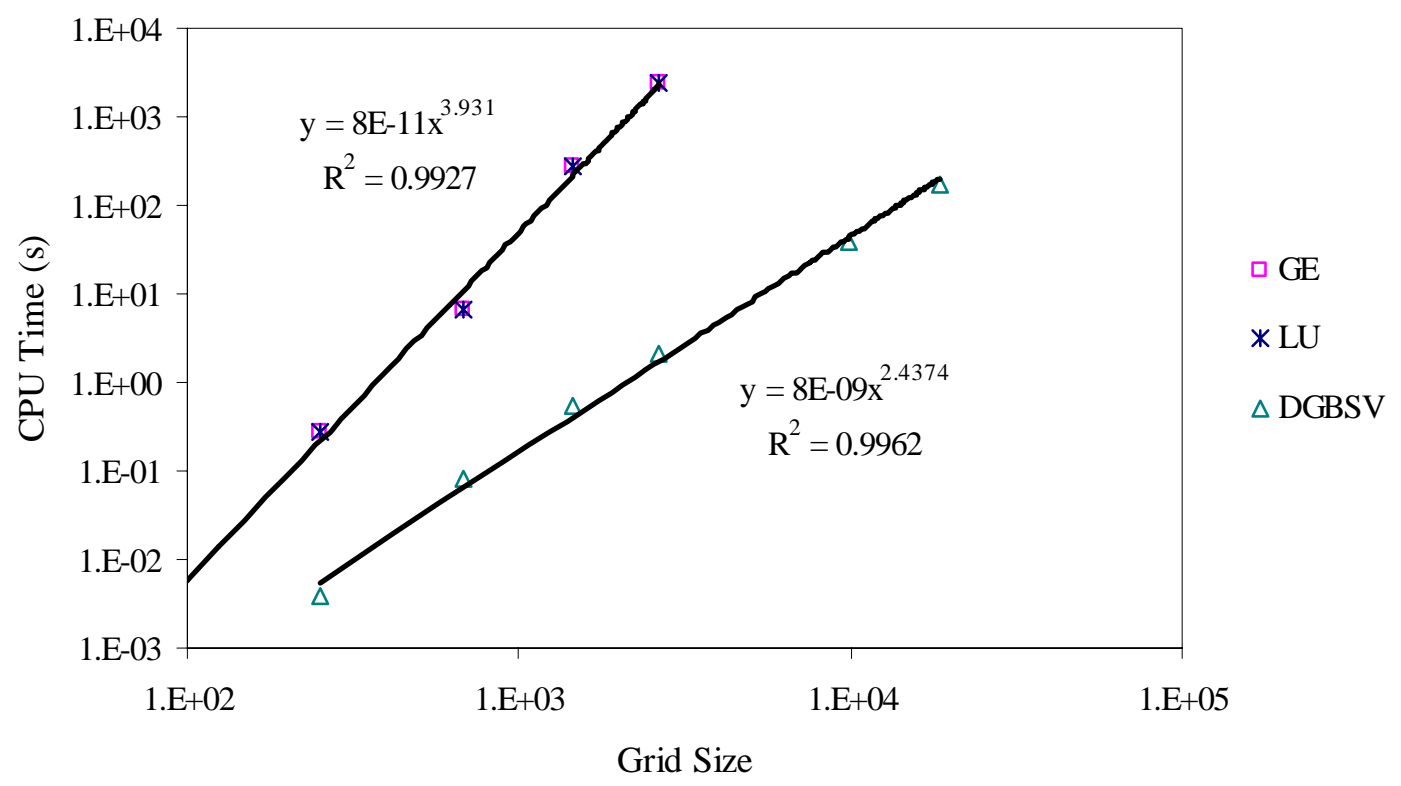

Figure 5. Direct Methods: Time Required for Convergence as a Function of Grid Size 
A power law fit of the relationship between time and grid size is included for the DGBSV solver. GE and LU data points are nearly coincident and the same power law fit is used to describe both. Indeed, these direct methods proved to work reasonably well on small grids, yet bigger grid sizes required large amounts of memory and numerous calculation steps. The direct methods eventually became impractical for the larger grids. For example, an 11x11x22 grid results in 11·11·22 = 2,262 equations in 2,262 unknowns and a full coefficient matrix with $(2,262)^{2}=7,086,244$ array elements. For the Gauss Elimination and Doolittle LU factorization programs, the next largest grid size considered, 17x17x34, has an input array of 96,550,276 elements and would have taken an estimated four and a half days to compute using $378,544 \mathrm{~K}$ of RAM. The banded solver from the LAPACK library, DGBSV, showed some improvement in both time and memory performance but still struggled on larger grids. The memory requirements for the direct methods are shown in Figure 6 with power law fits of the data for the DGBSV and LU/GE solver. These general trends were expected, yet the study was conducted for comparative purposes and to aid in understanding the solution process. Direct solutions could be preferable for obtaining the course-grid solutions for the new method. Again, since GE and LU memory requirements are so similar, the plotted points are essentially coincident and one trend line describes both. The left-most points are influenced by the finite amount of RAM that would be required even for a zero grid size, hence these points were excluded in the regression. 
Direct Methods: RAM Requirements vs Grid Size

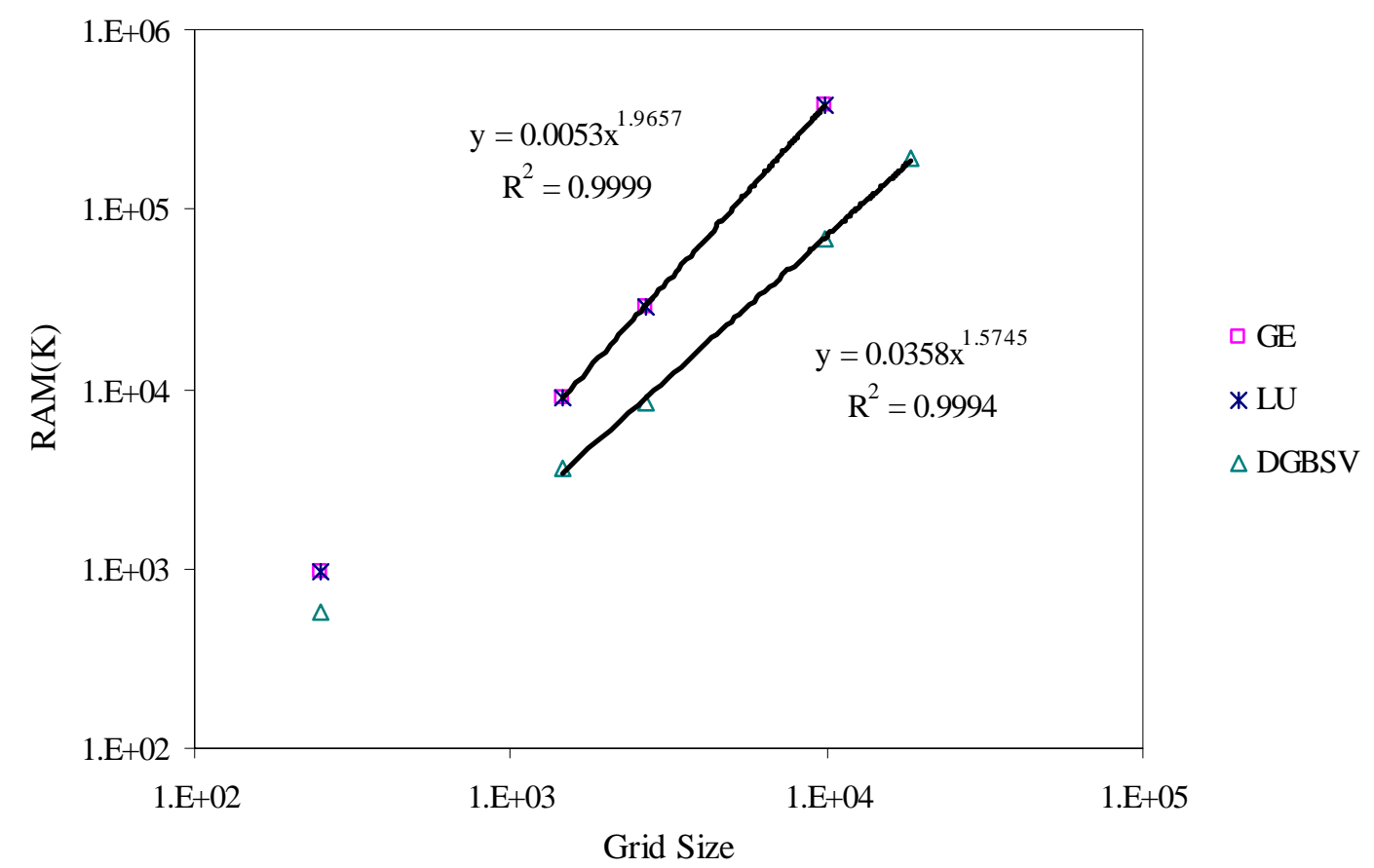

Figure 6. Direct Methods: Memory (RAM) Requirements as a Function of Grid Size

\subsection{Stationary and Nonstationary Iterative Methods: Results}

The iterative methods performance was tested at various grid sizes. Of all the iterative methods described in the previous chapter, LSOR was found to have the best convergence-time performance with SOR following closely. Of the five nonstationary MATLAB iterative methods, GMRES and LSQR were shown to have the best convergence-time performance. Figure 7 shows the convergence time performance of the various solvers as a function of grid size. Once again a power law fit is included for the best performer, LSOR. 
Itertative Methods: Time vs Grid Size

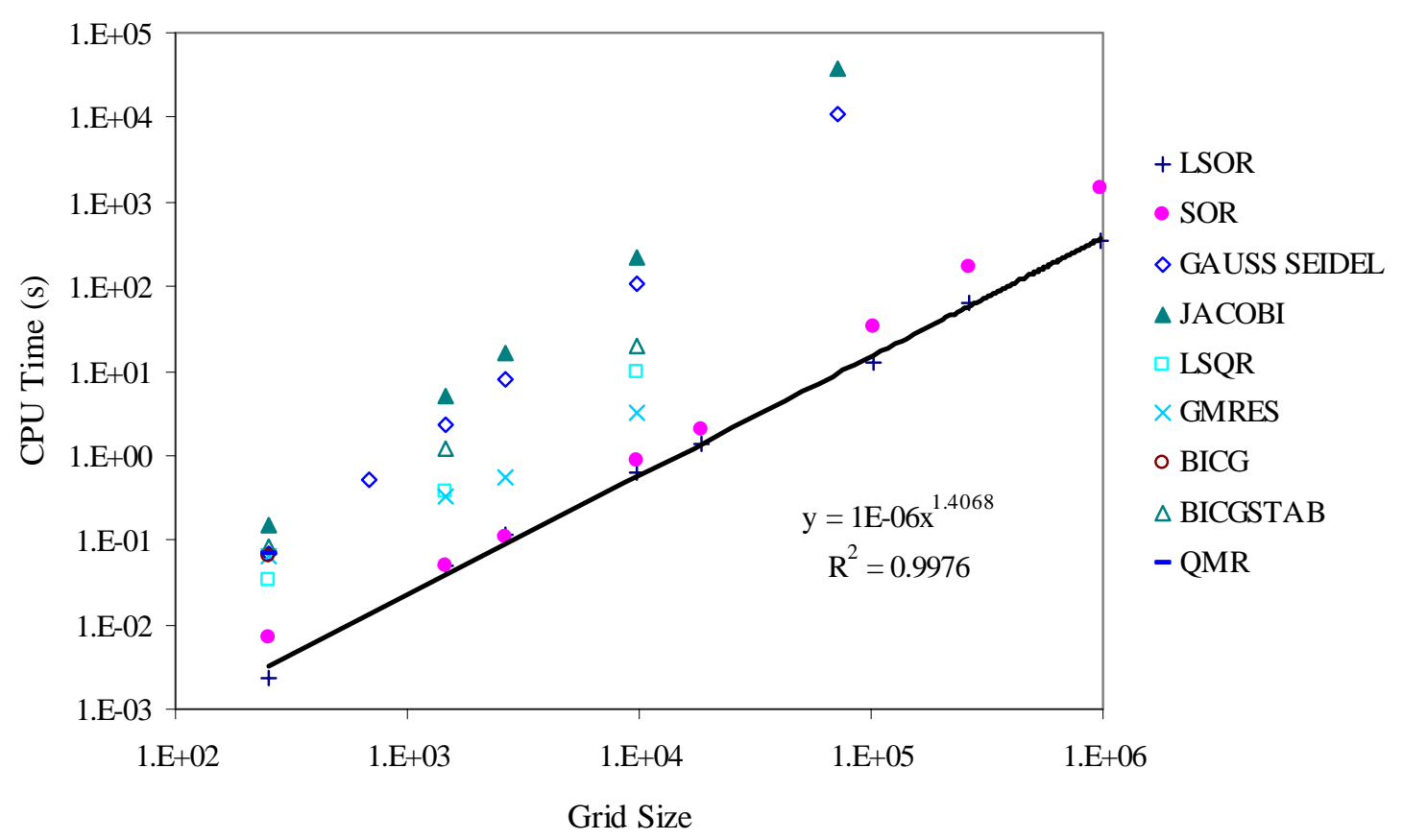

Figure 7. Iterative Methods: Time Required for Convergence as a Function of Grid Size

Extrapolation of the GMRES data from this plot suggests that GMRES could eventually perform better than any of the iterative methods at larger grid sizes. This can be seen by using the four data points for GMRES, which give a slope that indicates better performance for GMRES at larger grids; however GMRES memory requirements prohibited actual determination of results at larger grids. The potential of GMRES performing better than the other iterative methods at larger grids is somewhat diminished if the slope from the two largest grid sizes is used. Given the trends of the other iterative methods, decisions for GMRES were based on the data for the two largest grid sizes. QMR and BICG were stable for only the smallest grid size and had comparable convergence times to the other MATLAB iterative methods at the respective gird size. The use of a preconditioner at larger grid sizes could possibly help QMR and BICG to 
stabilize and generate reasonable answers, but this was not done as this study was conducted without preconditioners. BICGSTAB was shown to be the slowest of the five MATLAB iterative methods. As expected, Jacobi and Gauss Seidel iterative methods performed poorly with respect to the other methods when comparing convergence time.

SOR and LSOR performed better than all other iterative methods; LSOR had slightly better convergence times than SOR, but demanded more RAM than the other inhouse iterative methods for all grid sizes. It was difficult to determine how much memory was used for small grid sizes in MATLAB while the routines were in progress, yet for grid sizes larger than 17x17x34 the desktop computer did not have enough memory to complete the solution for any of the MATLAB methods. The results of the run-time memory performance of the various iterative methods are summarized in Figure 8. The values shown for SOR and LSOR are at the optimum over-relaxation factor, $\omega_{\text {opt }}$. Runtime memory requirements for MATLAB iterative methods at a grid size of $17 \times 17 \times 34$ were determined for the three methods that worked at this grid size. These three points, which show similar memory requirements for MATLAB methods, are shown in Figure 8 and indicate memory requirement two orders of magnitude larger than the stationary iterative methods.

This completes the first objective of this thesis, which was to test the performance of various solvers as a function of grid size. From these results, the best solver to be used on coarse and fine grids can be determined. 
Iterative Methods: Memory Requirements vs Grid Size

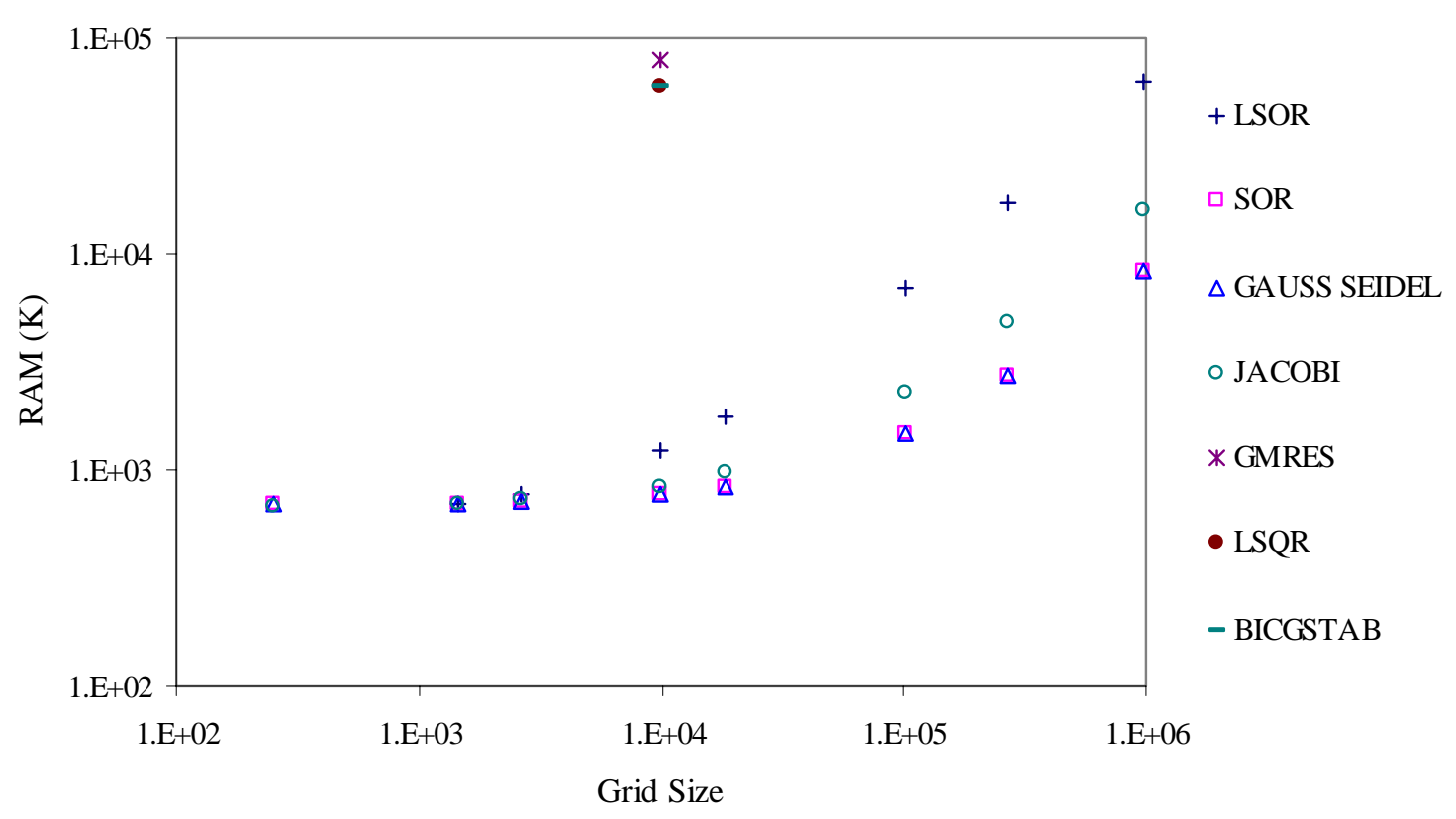

Figure 8. Iterative Methods: Memory (RAM) Requirements as a Function of Grid Size

\subsection{Summary}

Of the direct methods tested, the band solver, DGBSV, from the LAPACK library showed best performance with respect to both cpu time and memory requirements. LSOR showed the best cpu time performance of the stationary and nonstationary iterative methods, with SOR following closely behind; SOR and Gauss Seidel required the least amount of run-time memory of all iterative methods. These results will determine which solution algorithm should be used on both coarse and fine grids. 


\section{CHAPTER 5}

\section{DETERMINATION OF BEST SOLVER}

The second objective of this study was to analyze the previous results and determine which solver(s) performed best for coarse and fine grids. The best solver(s) would then be used in the nested-grid method. As the study of various solution methods was conducted, it became apparent which solution method operated best for coarse and fine grids and which would be easily implemented into the nested-grid method.

Of the direct methods considered, the band solver, DGBSV, from LAPACK

library was shown to perform best with respect to cpu time required for convergence and run-time memory requirements. For iterative methods, LSOR showed the best cpu time performance, with Successive-Over-Relaxation (SOR) following closely behind. In regard to memory requirements, SOR and Gauss Seidel required the same and least amount of memory for any of the iterative methods considered.

For the smallest grid sizes considered in this study, the cpu time and memory requirements for SOR were very similar to, but slightly larger than corresponding performance parameters of the band solver DGBSV. For grid sizes larger than the coarsest grid size, SOR performance was better than any of the direct methods considered.

With its convergence time performance similar to LSOR, its memory requirements much lower, and similar performance to the best direct method on the 
smallest grid considered, SOR became the primary candidate for the next phase of the study.

To ensure that SOR was the best solver for the task, work was done with the LSOR routine to implement the nested-grid method. The results showed little overall improvement to the speedup of the solution. This result was probably caused by the combination of direct and iterative methods incorporated into its solver routine. Due to memory constraints, the MATLAB iterative methods were not expected to perform well in the nested-grid method; however, the nested-grid method was implemented using GMRES and its performance recorded.

\subsection{Summary}

SOR was determined to be the best solver for the next phase of the study. It had similar convergence time to LSOR and its memory requirements were much lower. SOR had similar performance to the best direct method on the smallest grid size considered and outperformed any direct method on all larger grids. Even though their overall performance parameters were not as good as SOR, GMRES and LSOR were also considered for implementation in the nested-grid method. 


\section{CHAPTER 6}

\section{IMPLEMENTATION OF SOVER IN NESTED-GRID METHOD}

The third objective of this thesis was to implement the best solver in the nested-

grid solution method. In an effort to determine the potential advantage of the nested-grid solution method, a study was conducted in which selected pressure solutions were taken from a previously generated solution grid and inserted in matching locations from which they where taken, in an identical but unsolved grid. The pressure solution of the three dimensional grid was obtained again by iterating the system of equations to convergence, with the nested-grid pressures remaining constant throughout the iteration process. The performance improvement, or lack thereof, for the time required to reach convergence would be an indication of the future potential of applying Weber's accurate pressure solutions in a nested-grid manner. These results would also be used to determine optimal coarse grid sizes to be nested into various fine-grids.

\subsection{Grid Geometries and General Setup}

For the previous study of direct and iterative solver performance, various grid sizes were utilized as shown before in Table II, Chapter 3. For the nested-grid study, only fine-grid sizes that resulted in grid points coincident with course grids were used. Table III shows the fine grids used in the nested-grid study. 
Table III. Fine-Grid Sizes Used in Nested-Grid Study

\begin{tabular}{|c|c|}
\hline $\begin{array}{c}\text { Grid } \\
\text { Size }\end{array}$ & Grid Dimensions \\
\hline 250 & $5 \times 5 \times 10$ \\
\hline 1458 & $9 \times 9 \times 18$ \\
\hline 9826 & $17 \times 17 \times 34$ \\
\hline 71874 & $33 \times 33 \times 66$ \\
\hline 549250 & $65 \times 65 \times 130$ \\
\hline
\end{tabular}

For a given fine-grid size, the number of coarse-grid pressure points nested in the threedimensional array varied from a low concentration to a high concentration through various grid refinements. Table IV shows the level of grid refinement and the corresponding number of coarse-grid points nested into the fine-grid solution. The number of coarse-grid points includes the two wells.

Table IV. Course-Grid Sizes

\begin{tabular}{|c|c|}
\hline $\begin{array}{c}\text { Level of } \\
\text { Refinement }\end{array}$ & Coarse-Grid Size \\
\hline 1 & 18 \\
\hline 2 & 130 \\
\hline 3 & 1026 \\
\hline 4 & 8194 \\
\hline 5 & 65538 \\
\hline
\end{tabular}

The course-grid points were positioned equally distant from one another within the fine grid, and in the two reservoir halves they were symmetrical. For example, as shown in Figure 9, in a fine-grid plane determined by the height and width the number of fixed points at the first level of refinement was 4 , and in the plane determined from the height and length the number was 8 . In three dimensions, these numbers equated to a total of 16 fixed coarse-grid points plus the two wells for a total of 18 fixed grid points. 


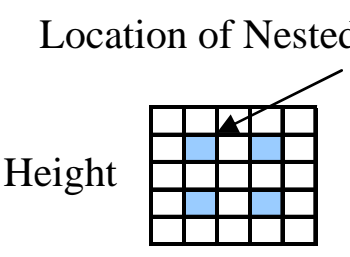

Width

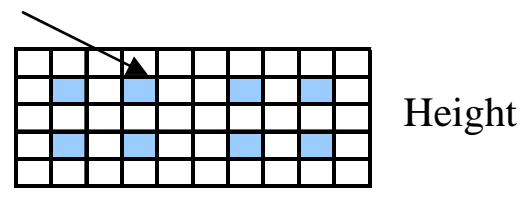

Length

Figure 9. Top and Side Plane Views of Fixed Points in a 5x5x10 Grid

Under the constraint that the nested-grid points, for any fine grid, be positioned equally distant from one another and symmetrically in each reservoir half, the following mathematical expression was determined to establish the number of nested-grid points at any level of coarse-grid refinement for the specified fine-grids.

$$
\text { Number of Coarse Grid Points }=2^{3 n+1}+2
$$

The mathematical expression indicates the total number of fixed points in the three dimensional simulation depending on the number of grid refinements " $n$ " desired; $n$ must be greater than or equal to one. In the case, where there are no additional nested-grid points, $n$ equals zero, expression (6-1) fails, and the two wells become the number of coarse-grid points. The greatest amount of coarse-grid refinement achievable depended on the fine grid in which the coarse grid was being placed. Refinement of the coarse grid was continued until, for the given fine-grid size, further refinement would result in the coarse grid not being aligned properly with the fine grid.

The values of the coarse-grid pressure solutions were imbedded into the fine grid such that the previous zero initial value of the fine grid was permanently replaced by the value of the coarse-grid pressure in the specified location throughout the iteration 
process. Table A.XII in Appendix A shows the coarse and fine grid sizes selected for the study, the number of fine-grid points between the course-grid points, and the percentage of coarse-grid points compared to the total number of fine-grid points. The number of coarse-grid points includes the two wells. The following figure shows the percentage of coarse-grid points in the nested-grid configurations used.

Nested-Grid Configurations: Percent Fixed Points

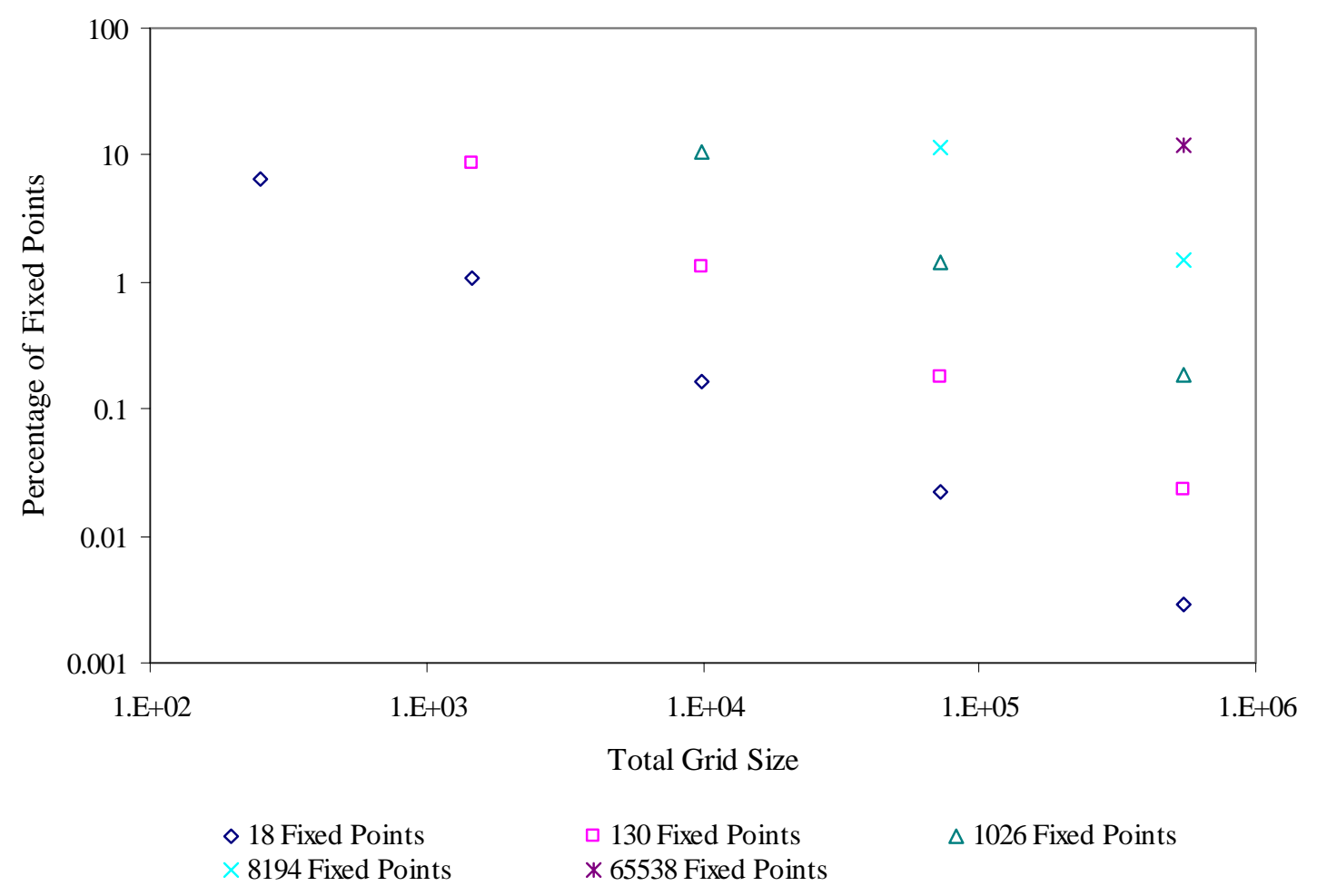

Figure 10. Nested-Grid Configurations: Percentage of Fixed Points

\subsection{Calculation of Coarse-Grid Pressure}

Successive-Over-Relaxation (SOR) was used to determine the coarse-grid pressure values at a pre-determined optimal over-relaxation factor. At this stage of the study, the goal was simply to demonstrate that the nested-grid solution method was 
feasible; consequently, Weber's solution was not used to generate the fine grid at this point. Instead, the coarse-grid pressures were extracted from a full fine-grid solution of LaPlace's equation using traditional finite-difference equations and generated at a convergence criterion of $10^{-9}$. This stringent criterion was selected so that errors in the extracted coarse grid would not influence the accuracy of the final fine-grid solution in which the coarse-grid points were inserted, and for which, the convergence criterion was $10^{-6}$.

The very accurate fine-grid solution values were used to represent pressures that would be obtained by using the new finite-difference equations that incorporate the physics of the flow. In practice, this method was taking selected accurate answers from the fine-grid solution to represent the coarse-grid values, and using them to generate the fine-grid solution again. This may be seen as using select parts of the answer to generate the answer again, yet this is exactly what the new finite-difference equations developed by Weber et al. permit. The new finite-difference equations allow a solution that is accurate on a coarse grid to be embedded into a finer grid to obtain, in a rapid manner, the final solution at fine-grid resolution.

\subsection{Calculation of Fine-Grid Pressure: LaPlace SOR Results}

With coarse-grid solutions determined, the accurate course-grid pressures were fixed into the fine-grid solution. For each of the nested-grid combinations of Table A.XII, an optimal over-relaxation factor, $\omega_{\mathrm{opt}}$, was determined, and the finely-gridded pressure solutions were generated at that value. Notable decreases in calculation times for the nested grids were observed. Table A.XIII in Appendix A shows the results. It tabulates 
$\omega_{\text {opt }}$, number of iterations required for convergence, time required for convergence, and ratio of time per iteration for the various nested-grid setups. In the table, $\mathrm{N}_{\mathrm{FG}}$ refers to the total number of fine-grid points and $\mathrm{N}_{\mathrm{CG}}$ refers to the number of coarse-grid points nested into a given fine grid. The following figure shows the time required for convergence as a function of total grid size for the various nested-grid configurations.

\section{LaPlace SOR: Time Required for Convergence}

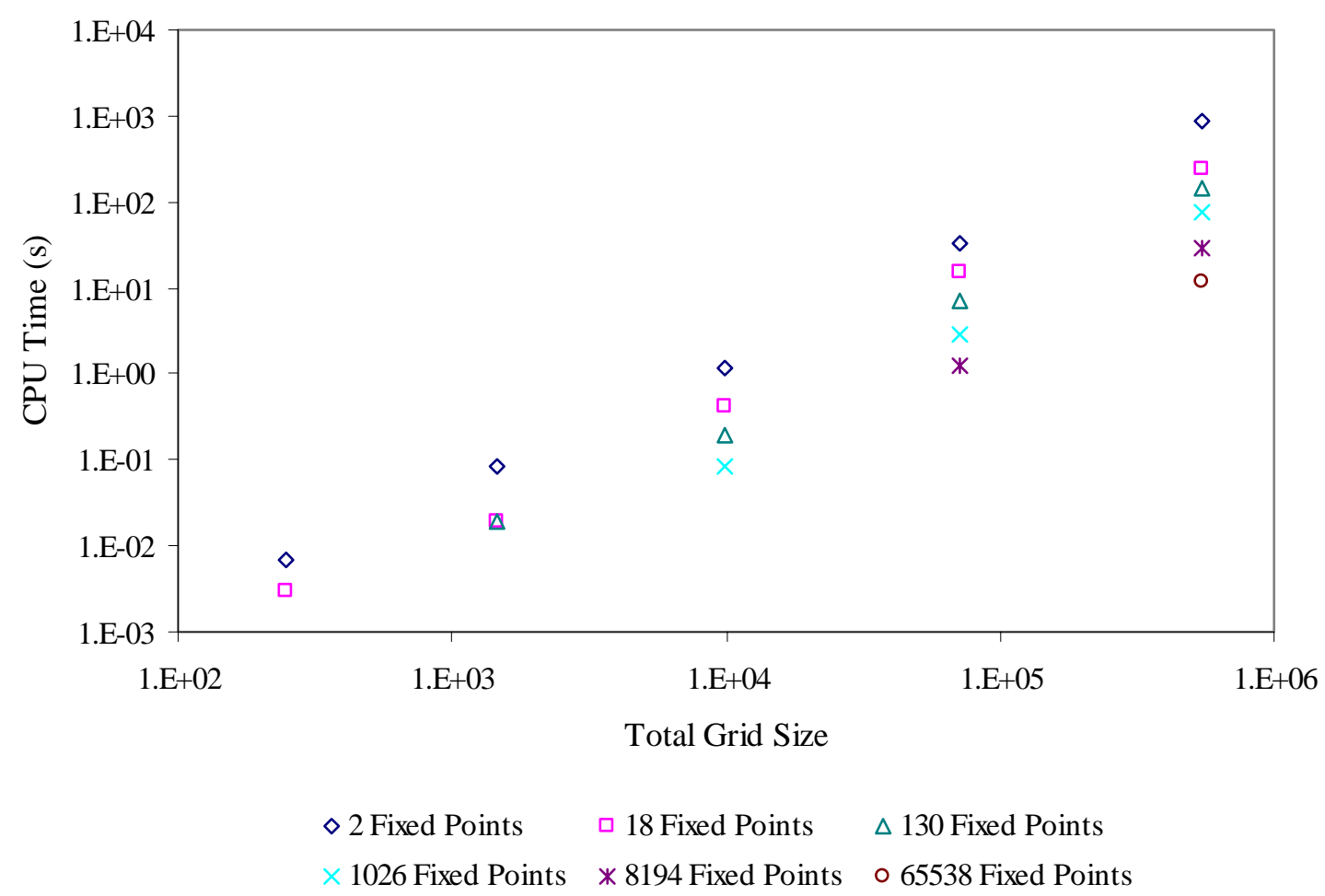

Figure 11. LaPlace SOR: Time Required for Convergence of Various Nested-Grids

\subsection{Nested-Grid Method: GMRES Results}

Although GMRES did not appear from the previous studies to have the potential of being the best solver to use in the nested-grid method, it was investigated nonetheless because of its recommendation by industry as a state-of-the-art solver. As noted earlier, 
all of the MATLAB iterative functions used large amounts of memory that made the solution of large grids impossible on the desktop computer being used. For this nestedgrid study, the same fine-grid sizes as shown in Table III were used. Course-grid points were spaced equally distant from one another within the fine grid, and in the two reservoir halves they were symmetrical. The pressure values of the coarse-grid points were taken from a previously generated fine-grid solution as described earlier. GMRES does not require an optimal over-relaxation factor.

The results of the fine and nested-grid study for GMRES are shown in Table A.XIV of Appendix A. The following figure shows the time required for Convergence as a function of total grid size for GMRES.

GMRES: Time Required for Convergence

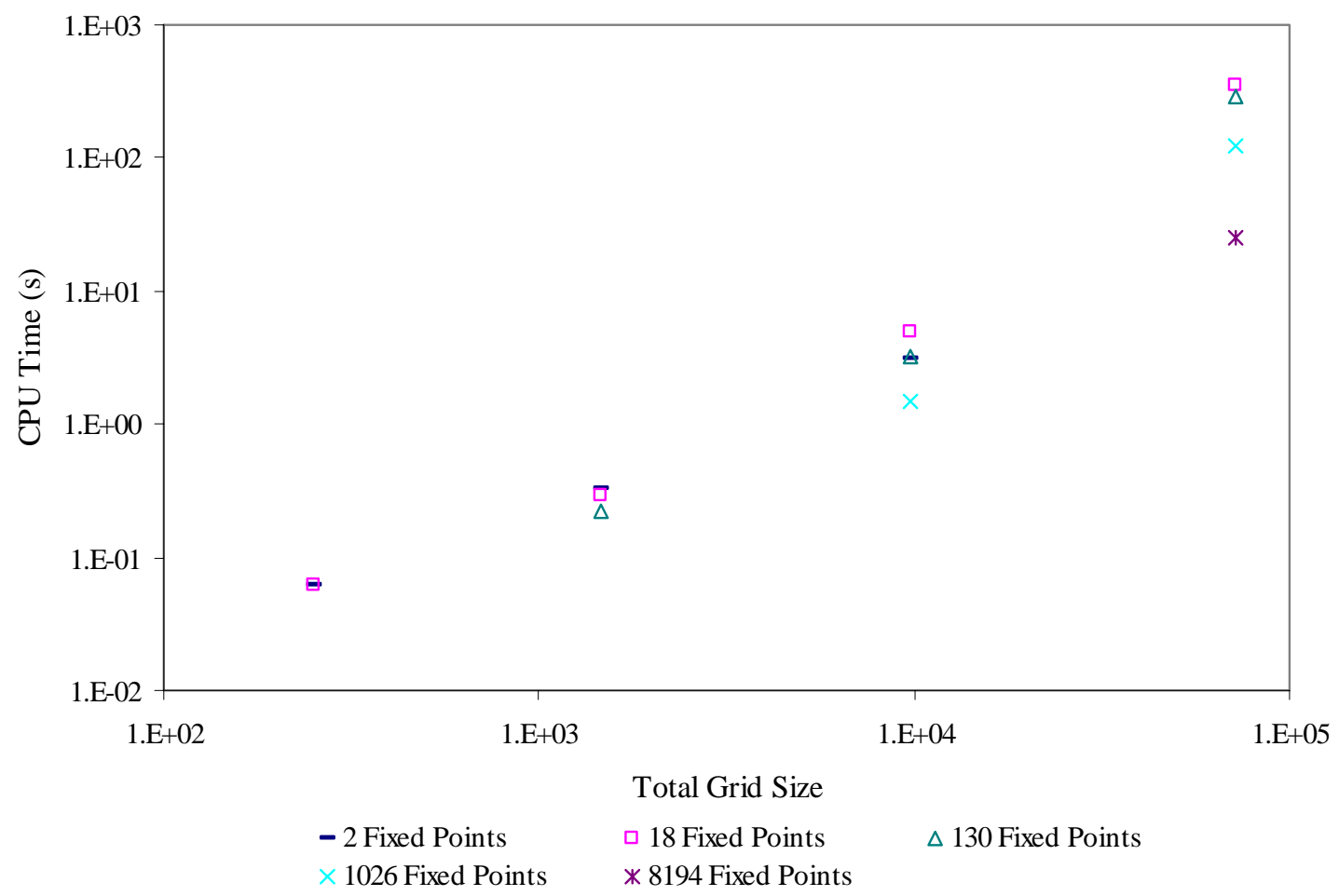

Figure 12. GMRES: Time Required for Convergence as a Function of Grid Size 
Figure 12 reveals an interesting consequence of imbedding fixed points in the solution method. It can be seen that the original fine-grid solution (2 fixed points) was only achieved up to 9,826 fine-grid points. Grid sizes above this required too much memory to successfully determine a solution on the desktop computer. As fixed points were added to the fine grid, according to the coarse-grid refinement method, the previously unattainable solutions became attainable. The finely-gridded pressures for a grid size of 33x33x66 (71,874 fine-grid points) was achieved by applying the nested-grid method using the same desktop computer. This result highlights the potential of the new method to compute reservoir simulation pressures for more refined grids on the same computer. However, even using the nested-grid method, the 65x65x130 grid was still too large for GMRES on the desktop computer.

\subsection{Data Analysis}

The data recorded in Tables A.XIII and A.XIV were used to determine the optimal number of fixed-coarse-grid points that should be embedded in a desired fine grid to obtain the pressure solution for any nested-grid setup in the least amount of time. This was done by first regressing the results in Tables A.XIII and A.XIV to obtain mathematical expressions to predict both $\omega_{\text {opt }}$ (for SOR only) and the number of iterations $\left(\mathrm{N}_{\text {Iter }}\right)$ required for convergence, for any nested-grid setup. Both of the expressions were functions of the number of fixed-coarse-grid points $\left(\mathrm{N}_{\mathrm{CG}}\right)$ and the total number of fine-grid points $\left(\mathrm{N}_{\mathrm{FG}}\right)$. These results were then used in a dimensionless time analysis which predicted the performance of any nested-grid arrangement using the functions generated for $\mathrm{N}_{\text {iter }}$. Preliminary analysis of these results is recorded in Appendix 
B and was reported in the Canadian International Petroleum Conference Paper 2005112. ${ }^{33}$ The results showed a significant improvement was achieved by implementation of the nested-grid algorithm for LaPlace SOR and GMRES. These results became the foundation of the study that was done later when Weber's equations were applied in the solution algorithm. Later in the study, a better regression for all of the data, including the data collected when Weber's equations were incorporated into the nested-grid algorithm, was obtained and applied in the dimensionless time analysis. These results are reported later in the thesis in Chapters 10 and 11.

\subsection{Summary}

The nested-grid method was implemented using various nested-grid configurations. LaPlace SOR and GMRES were used to calculate the finely-gridded pressures on the nested-grid configurations and performance data was recorded (See Tables A.XIII and A.XIV). Significant improvements in the time required for convergence were noted for both LaPlace SOR and GMRES, as nested-pressures were fixed in the fine-grid. These improvements gave a strong indication that Weber's accurate pressure-solutions could be implemented in a nested-grid method and reduce computation time required to achieve finely-gridded reservoir simulation pressures. 


\section{CHAPTER 7}

\section{INTERPOLATION}

\subsection{Considering Interpolation}

Before Weber's equations were implemented, the fourth objective of the study, which was to consider the possible advantages of interpolation, needed to be satisfied. As mentioned previously in Chapter 3, iterative methods require an initial approximation of the solution to begin the iterative process. In an effort to speed up the solution method further, a three-dimensional linear interpolation of the nested-grid configuration was computed. After interpolation was completed, the finely-gridded pressure solution was achieved by iterating to convergence using SOR and the nested-grid method described in Chapter 6. If interpolation proved to be worthwhile it would be incorporated later in the study with Weber's equations. Previously, as described in Chapter 6, the starting pressure values for the calculation of a fine-pressure grid were the pressure values of any nestedgrid points, the pressure values of the wells, and all remaining pressure values were zero. By interpolating the values of the accurate nested-grid points over the zero starting values of the other grid points, the solution convergence should accelerate. The interpolation program came from the literature, where a two dimensional example was extended to three dimensions. ${ }^{34}$ The essential equation to the interpolation program in three dimensions is as follows. 


$$
\begin{aligned}
& \mathrm{P}(\mathrm{I}, \mathrm{J}, \mathrm{K})= \\
& \mathrm{P}(1) *(1-\mathrm{Fx}) *(1-\mathrm{Fy}) *(1-\mathrm{Fz})+ \\
& \mathrm{P}(2) * \mathrm{Fx} *(1-\mathrm{Fy}) *(1-\mathrm{Fz})+ \\
& \mathrm{P}(3) * \mathrm{Fx} * \mathrm{Fy} *(1-\mathrm{Fz})+ \\
& \mathrm{P}(4) *(1-\mathrm{Fx}) * \mathrm{Fy} *(1-\mathrm{Fz})+ \\
& \mathrm{P}(5) *(1-\mathrm{Fx}) *(1-\mathrm{Fy}) * \mathrm{Fz}+ \\
& \mathrm{P}(6) * \mathrm{Fx} *(1-\mathrm{Fy}) * \mathrm{Fz}+ \\
& \mathrm{P}(7) * \mathrm{Fx} * \mathrm{Fy} * \mathrm{Fz}+ \\
& \mathrm{P}(8) *(1-\mathrm{Fx}) * \mathrm{Fy} * \mathrm{Fz}
\end{aligned}
$$

$\mathrm{P}(1)$ through $\mathrm{P}(8)$ are the course-grid block pressures. Fx, Fy, and Fz represent the fraction of $\mathrm{x}, \mathrm{y}$, and z-distances that the fine-grid points are from point (1) of the coursegrid block. For example, if the new grid point is exactly in the middle of eight existing grid points, i.e. at the old cell corners, $\mathrm{Fx}=\mathrm{Fy}=\mathrm{Fz}=0.5$. Note that even for this simple linear interpolation, twenty-four multiplications and three divisions are required for each interpolated value. The interpolation is much more computer time intensive than the SOR iterations.

To obtain the final fine-grid pressure solution using interpolation, the nested-grid pressure values were interpolated and the resulting grid was iterated to convergence with the values of specified nested-grid points remaining fixed throughout. Table A.XV in Appendix A shows the results of the interpolation study. Table A.XVI in Appendix A shows the total time required to obtain, at the same $\omega_{\text {opt }}$, the fine-grid pressure solution with interpolation, the time required to obtain the fine-grid pressure solution without interpolation, and the resulting ratio. The following figure shows how the time required to obtain the full fine-grid solution with and without interpolation for the various nestedgrid configurations compare. 


\section{Comparison of Time Required to Obtian Full Fine-Grid Pressure \\ Solution With and Without Interpolation}

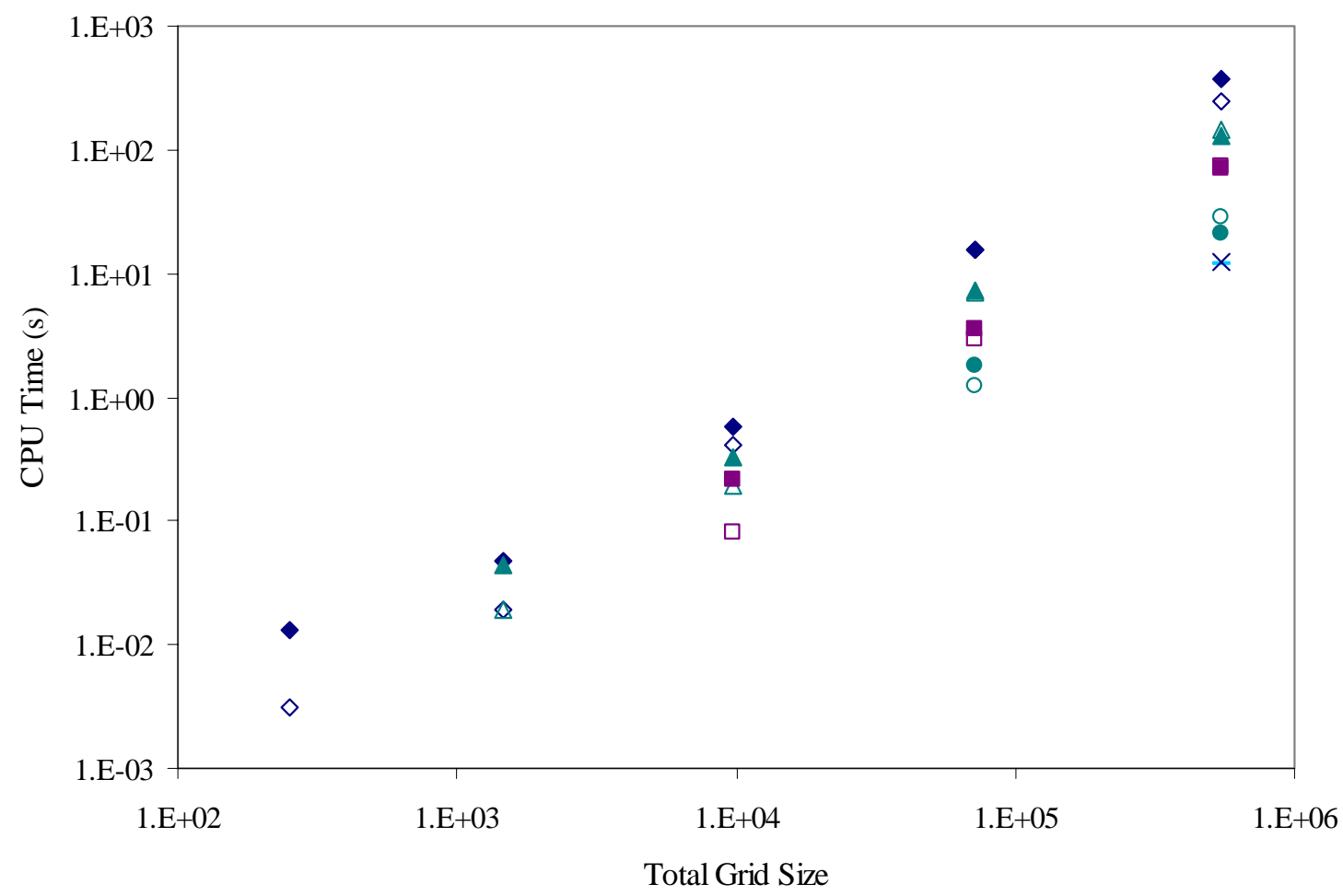

- 18 Fixed Points with Interpolation 130 Fixed Points with Interpolation - 1026 Fixed Points with Interpolation - 8194 Fixed Points with Interpolation - 65538 Fixed Points with Interpolation $\diamond 18$ Fixed Points without Interpolation

$\triangle 130$ Fixed Points without Interpolation

$\square 1026$ Fixed Points without Interpolation

○ 8194 Fixed Points without Interpolation

$\times 65538$ Fixed Points without Interpolation

Figure 13. Comparison of Time Required to Obtain Full Fine-Grid Pressure Solution With and Without Interpolation

As shown in Figure 13, the results of the interpolation program did not show a significant amount of improvement. It was evident in many cases that the time required to generate a fine-grid pressure solution using interpolation took longer than the time required to simply iterate the nested-grid algorithm to convergence. The values in the last column of Table A.XVI, are ratios of the time required to linearly interpolate the finegrid solution and then iterate it to convergence, divided by the iteration time required 
when interpolation was not used. The ratios indicate that larger fine-grids benefited the most from the interpolation algorithm.

\subsection{Summary}

In an effort to speed up the solution method further, a three-dimensional linear interpolation of the nested-grid configuration was computed. Interpolation was not found to reduce the cpu time required to obtain the finely-gridded pressures in a significant manner. The lack of significant improvement using interpolation emphasizes the impact that the optimal over-relaxation factor has on the solution of a system of equations. It was thought that the interpolation program would possibly improve the solution method, yet for interpolation in three dimensions it became clear that the time required to simply interpolate values for every grid point added enough time to the overall solution method to make it an undesirable algorithm step. For this reason, interpolation was not recommended for further use in the study. 


\section{CHAPTER 8}

\section{WEBER'S COEFFICIENTS}

With validation that the nested-grid method could be applied successfully and that interpolation was not beneficial, the next objective of successfully applying Weber's

coefficients in the nested-grid solution method was pursued. Dan Weber et al. ${ }^{27}$ considered finite-difference equations for reservoir pressures based on two new functional forms: $\Sigma \ln (\mathrm{r})$ and $\Sigma 1 / \mathrm{r}$; where r represents the distance to each of the wells. The $\ln (r)$ formulation was used to determine pressures from straight-line wells and the $1 / \mathrm{r}$ form was used to represent pressures from point sources. Weber et al. determined that for finite-difference equations based on inverse-r functions, it was possible to reduce the pressure errors compared to traditional polynomial based finite-difference formulations by a factor of 10,071 using a finite-volume formulation. ${ }^{27}$ According to Weber et al., the most accurate pressure solutions are achieved with a volumetric approach in which the flux varied throughout the finite difference grid cell as prescribed by the following equation.

$$
p=a \sum_{n}^{\substack{\text { all } \\ \text { wells }}} \frac{Q_{n}}{r_{n}}+c
$$

$Q_{n}$ is the volumetric injection rate of well $n, r_{n}$ is the distance to well $n, c$ is the integration constant, $p$ is the pressure, and $a$ is a proportionality constant. Darcy's law, in 
which $q$ is the flux of fluid through the porous media, $K$ is permeability, and $\mu$ is fluid viscosity is as follows.

$$
q=-\frac{K}{\mu} \frac{d p}{d r}
$$

Equation (8-1) and (8-2) were used to determine the total flux through various faces of the grid cell being analyzed. As an example, the following equation determines the total flux through one of the $\mathrm{x}$-faces of a given cell:

$$
Q_{x}=-\frac{K_{x}}{\mu} \int \frac{d p}{d x} d A=a \frac{K_{x}}{\mu} \int_{y_{j}-\frac{\Delta y}{2}}^{y_{j}+\frac{\Delta y}{2}} \int_{z_{k}-\frac{\Delta z}{2}}^{z_{k}+\frac{\Delta z}{2}} \frac{x d z d y}{\sqrt{\left(x^{2}+y^{2}+z^{2}\right)^{3}}}
$$

The double integral in Equation (8-3) includes the solid angle, $\Omega$, subtended by the cell face relative to the well point source. A surface at a point is defined to be the surface area of the projection of that surface onto a unit sphere centered at that point. In other words, the solid angle is $4 \pi$ times the ratio of the area of the face projected on a sphere of unit radius, centered at the well, to the entire area of the sphere. The completed double integration in equation (8-3), results in the following expression.

$$
\begin{aligned}
& \Omega_{x}=\tan ^{-1}\left[\frac{\left(z+\frac{\Delta z}{2}\right)\left(y+\frac{\Delta y}{2}\right)}{x \sqrt{x^{2}+\left(y+\frac{\Delta y}{2}\right)^{2}+\left(z+\frac{\Delta z}{2}\right)^{2}}}\right]-\tan ^{-1}\left[\frac{\left(z-\frac{\Delta z}{2}\right)\left(y+\frac{\Delta y}{2}\right)}{x \sqrt{x^{2}+\left(y+\frac{\Delta y}{2}\right)^{2}+\left(z-\frac{\Delta z}{2}\right)^{2}}}\right]- \\
& \tan ^{-1}\left[\frac{\left(z+\frac{\Delta z}{2}\right)\left(y-\frac{\Delta y}{2}\right)}{x \sqrt{x^{2}+\left(y-\frac{\Delta y}{2}\right)^{2}+\left(z+\frac{\Delta z}{2}\right)^{2}}}\right]+\tan ^{-1}\left[\frac{\left(z-\frac{\Delta z}{2}\right)\left(y-\frac{\Delta y}{2}\right)}{x \sqrt{x^{2}+\left(y-\frac{\Delta y}{2}\right)^{2}+\left(z-\frac{\Delta z}{2}\right)^{2}}}\right]
\end{aligned}
$$


The proportionality constant, $a$, in Equation (8-3) was determined by subtracting the pressures at adjacent grid points, which results in the following equation in terms of the pressures $\mathrm{p}_{\mathrm{i}+1}$ and $\mathrm{p}_{\mathrm{i}}$ :

$$
a=\frac{p_{i+1}-p_{i}}{\sum_{n} \frac{Q_{n}}{r_{n, i+1}}-\sum_{n} \frac{Q_{n}}{r_{n, i}}}
$$

With these equations established, the total flux through the cell's $\mathrm{x}$-face to the right of the cell center becomes.

$$
Q_{x+}=\frac{K_{x+}}{\mu} \frac{\left(p_{i+1}-p_{i}\right) \sum Q \Omega_{x+}}{\sum \frac{Q}{r_{i+1}}-\sum \frac{Q}{r_{i}}}
$$

Hence traditional finite difference equations, such as those used previously in the study, can be used for the Weber formulations if the usual permeability $\mathrm{K}$ is replace by the pseudo-permeability, $\mathrm{K}^{\prime}$, as shown in the following equation.

$$
K_{x+}^{\prime}=K_{x+} \frac{\sum Q \Omega_{x+}}{\sum \frac{Q}{r_{i+1}}-\sum \frac{Q}{r_{i}}}
$$

Weber recommended that in the implementation of his equations, pseudopermeability coefficients be calculated for the 18 interior faces in each direction of the 27 cells immediately surrounding and including the well. The remaining grid points maintained their physically assigned permeability and did not need to be corrected by Weber's equations.

To understand how these equations were implemented into discretized space, the following explanation is given. Figure 10 shows a two dimensional $5 \times 5$ view of a plane that includes a well for a given $5 \times 5 \times 10$ reservoir which is centered at grid point $i_{w}, j_{w}, k_{w}$. 
In this two dimensional slice of the reservoir, nine of the 27 cells immediately surrounding the well are represented with their corresponding cell faces in negative and positive directions. Coefficients are calculated for the 18 interior cell faces in each direction; the coefficients across any interface between two grid cells must be consistent to preserve continuity.

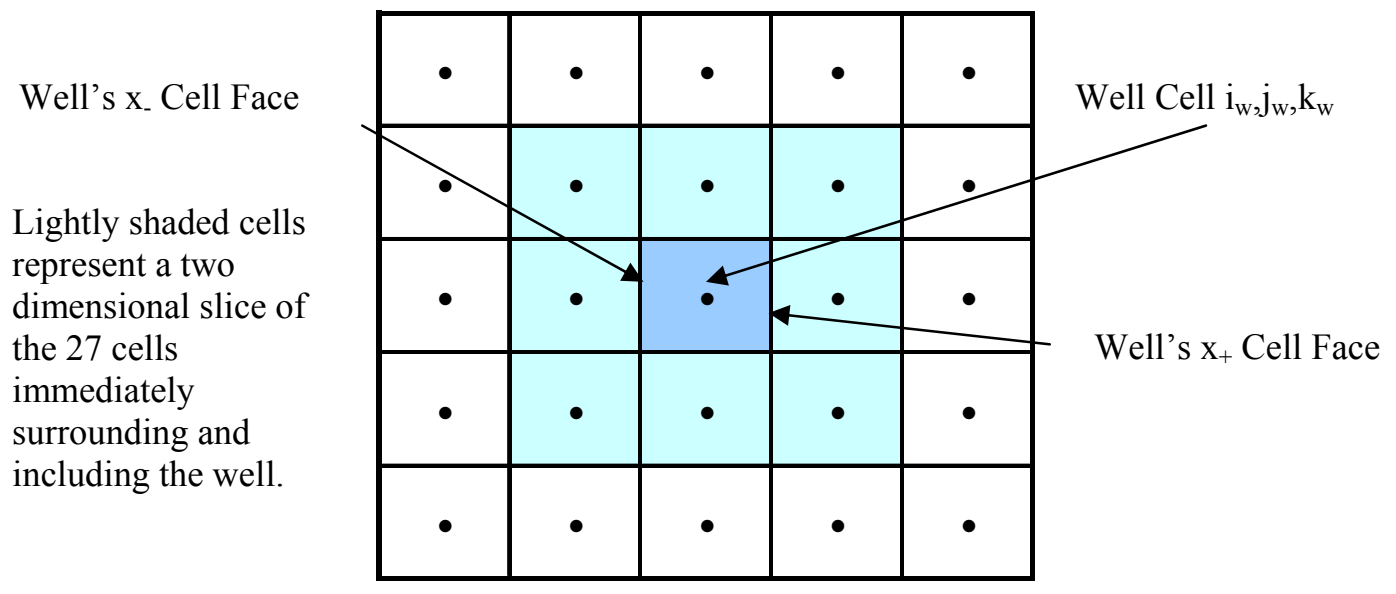

Figure 14. Application of Weber's Coefficients

The spacing between the various grid points in each of the three dimensions is defined as follows, but any values could be used.

$$
\Delta x=1 \quad \Delta y=1 \quad \Delta z=1
$$

The distances from a well to the various grid points and their corresponding cell faces are given by the following equations which only apply to equally spaced grids:

For the two $x$-faces (the cell faces perpendicular to the $x$ axis) of the cell $(i, j, k)$

$$
x_{+}=\left(i-i w+\frac{1}{2}\right) \Delta x \quad x_{-}=\left(i-i w-\frac{1}{2}\right) \Delta x
$$


The corresponding $\mathrm{y}$ and $\mathrm{z}$ distances are defined by the following equations.

$$
y=(j-j w) \Delta y \quad z=(k-k w) \Delta z
$$

For the two y-faces (the cell faces perpendicular to the $y$ axis) of the cell (i,j,k)

$$
y_{+}=\left(j-j w+\frac{1}{2}\right) \Delta y \quad y_{-}=\left(j-j w-\frac{1}{2}\right) \Delta y
$$

The corresponding $\mathrm{x}$ and $\mathrm{z}$ distances are defined by the following equations.

$$
x=(i-i w) \Delta x \quad z=(k-k w) \Delta z
$$

For the two z-faces (the cell faces perpendicular to the $z$ axis of the cell (i,j,k)

$$
z_{+}=\left(k-k w+\frac{1}{2}\right) \Delta z \quad z_{-}=\left(k-k w-\frac{1}{2}\right) \Delta z
$$

The corresponding $\mathrm{x}$ and $\mathrm{y}$ distances are defined by the following equations.

$$
x=(i-i w) \Delta x \quad y=(j-j w) \Delta y
$$

With the above distances defined, the solid angle could be calculated for the various cell faces of a given grid block located at a specified distance from the well. The following equations show the mathematical expressions used to calculate the solid angle for the positive and negative face of the cell in the $\mathrm{x}$ direction. Similar expressions were used in the $\mathrm{y}$ and $\mathrm{z}$ directions. 
For the two $x$-faces (the cell faces perpendicular to the $x$ axis) of the cell $(i, j, k)$

$\Omega_{x+}=\tan ^{-1}\left[\frac{\left(z+\frac{\Delta z}{2}\right)\left(y+\frac{\Delta y}{2}\right)}{x_{+} \sqrt{x_{+}{ }^{2}+\left(y+\frac{\Delta y}{2}\right)^{2}+\left(z+\frac{\Delta z}{2}\right)^{2}}}\right]-\tan ^{-1}\left[\frac{\left(z-\frac{\Delta z}{2}\right)\left(y+\frac{\Delta y}{2}\right)}{x_{+} \sqrt{x_{+}^{2}+\left(y+\frac{\Delta y}{2}\right)^{2}+\left(z-\frac{\Delta z}{2}\right)^{2}}}\right]-$
$\tan ^{-1}\left[\frac{\left(z+\frac{\Delta z}{2}\right)\left(y-\frac{\Delta y}{2}\right)}{x_{+} \sqrt{x_{+}{ }^{2}+\left(y-\frac{\Delta y}{2}\right)^{2}+\left(z+\frac{\Delta z}{2}\right)^{2}}}\right]+\tan ^{-1}\left[\frac{\left(z-\frac{\Delta z}{2}\right)\left(y-\frac{\Delta y}{2}\right)}{x_{+} \sqrt{x_{+}{ }^{2}+\left(y-\frac{\Delta y}{2}\right)^{2}+\left(z-\frac{\Delta z}{2}\right)^{2}}}\right]$

$\Omega_{x^{-}}=\tan ^{-1}\left[\frac{\left(z+\frac{\Delta z}{2}\right)\left(y+\frac{\Delta y}{2}\right)}{x_{-}{\sqrt{x_{-}{ }^{2}+\left(y+\frac{\Delta y}{2}\right)^{2}+\left(z+\frac{\Delta z}{2}\right)^{2}}}^{2}}\right]-\tan ^{-1}\left[\frac{\left(z-\frac{\Delta z}{2}\right)\left(y+\frac{\Delta y}{2}\right)}{x_{-} \sqrt{x_{-}^{2}+\left(y+\frac{\Delta y}{2}\right)^{2}+\left(z-\frac{\Delta z}{2}\right)^{2}}}\right]-$

$\tan ^{-1}\left[\frac{\left(z+\frac{\Delta z}{2}\right)\left(y-\frac{\Delta y}{2}\right)}{x_{-} \sqrt{x_{-}^{2}+\left(y-\frac{\Delta y}{2}\right)^{2}+\left(z+\frac{\Delta z}{2}\right)^{2}}}\right]+\tan ^{-1}\left[\frac{\left(z-\frac{\Delta z}{2}\right)\left(y-\frac{\Delta y}{2}\right)}{x_{-} \sqrt{x_{-}^{2}+\left(y-\frac{\Delta y}{2}\right)^{2}+\left(z-\frac{\Delta z}{2}\right)^{2}}}\right]$

In addition to the solid angles computed, the various radial distances from the well are also needed before the pseudo-permeability can be determined. The equations used to determine the radial distance of a given point from a well are as follows. 
Values of $\mathbf{r}$ to be used in pseudo-permeability calculation for cell $(i, j, k)$ in $\mathbf{x}$ direction

$$
\begin{array}{ccr}
x_{+}=(i-i w+1) \Delta x & z=(k-k w) \Delta z \\
r_{x+1} & =\sqrt{{x_{+}{ }^{2}+y^{2}+z^{2}}} & \\
X_{-}=(i-i w-1) \Delta x & =(j-j w) \Delta y & z=(k-k w) \Delta z \\
r_{x-1} & =\sqrt{{x_{-}}^{2}+y^{2}+z^{2}} &
\end{array}
$$

Values of $\mathbf{r}$ to be used in pseudo-permeability calculation for cell $(i, j, k)$ in y direction

$$
\begin{aligned}
& x=(i-i w) \Delta x \quad y_{+}=(j-j w+1) \Delta y \quad z=(k-k w) \Delta z \\
& r_{y+1}=\sqrt{x^{2}+y_{+}^{2}+z^{2}} \\
& x=(i-i w) \Delta x \quad y_{-}=(j-j w-1) \Delta y \quad z=(k-k w) \Delta z \\
& r_{y-1}=\sqrt{x^{2}+y_{-}^{2}+z^{2}}
\end{aligned}
$$

Values of $\mathbf{r}$ to be used in pseudo-permeability calculation for cell $(\mathbf{i}, \mathbf{j}, \mathbf{k})$ in $\mathrm{z}$ direction

$$
\begin{array}{rlrl}
x=(i-i w) \Delta x & y & =(j-j w) \Delta y & \\
r_{z+1} & =\sqrt{x^{2}+y^{2}+z_{+}{ }^{2}} & \\
x=(i-i w) \Delta x & y & =(j-j w) \Delta y & z_{+}=(k-k w+1) \Delta z \\
r_{z-1} & =\sqrt{x^{2}+y^{2}+z_{-}^{2}}
\end{array}
$$

The radial distance " $r$ " from the well to any particular point is given by

$$
x=(i-i w) \Delta x \quad y=(j-j w) \Delta y \quad z=(k-k w) \Delta z
$$




$$
r=\sqrt{x^{2}+y^{2}+z^{2}}
$$

With the distances, solid angles and radial distances calculated, it was possible to determine the pseudo-permeability $\mathrm{K}^{\prime}$ using Equation (8-7). To incorporate the pseudopermeabilities into the mathematical expression used to calculate the pressure for a grid point, the ratio $(\lambda)$ of pseudo-permeability to actual permeability had to be calculated.

The mathematical expressions for lambda values in all directions are shown below.

Lambda calculations for the two $x$-faces of the cell $(\mathbf{i}, \mathbf{j}, \mathbf{k})$

$$
\lambda_{x-}=\frac{\sum Q \Omega_{x-}}{\sum \frac{Q}{r_{x-1}}-\sum \frac{Q}{r}} \quad \lambda_{x+}=\frac{\sum Q \Omega_{x+}}{\sum \frac{Q}{r_{x+1}}-\sum \frac{Q}{r}}
$$

Lambda calculations for the two $y$-faces of the cell $(i, j, k)$

$$
\lambda_{y-}=\frac{\sum Q \Omega_{x-}}{\sum \frac{Q}{r_{y-1}}-\sum \frac{Q}{r}} \quad \lambda_{y+}=\frac{\sum Q \Omega_{x+}}{\sum \frac{Q}{r_{y+1}}-\sum \frac{Q}{r}}
$$

Lambda calculations for the two $y$-faces of the cell $(\mathbf{i}, \mathbf{j}, \mathbf{k})$

$$
\lambda_{z-}=\frac{\sum Q \Omega_{x-}}{\sum \frac{Q}{r_{z-1}}-\sum \frac{Q}{r}} \quad \lambda_{z+}=\frac{\sum Q \Omega_{x+}}{\sum \frac{Q}{r_{z+1}}-\sum \frac{Q}{r}}
$$

From these lambda coefficients, the values of pressure for the grid cells influenced by Weber's coefficients could be calculated by the following equation. 


$$
P=\frac{\lambda_{x-} P_{x-1}+\lambda_{x+} P_{x+1}+\lambda_{y-} P_{y-1}+\lambda_{y+} P_{y+1}+\lambda_{z-} P_{z-1}+\lambda_{z+} P_{z+1}}{\lambda_{x-}+\lambda_{x+}+\lambda_{y-}+\lambda_{y+}+\lambda_{z-}+\lambda_{z+}}
$$

For the idealized reservoir in this study, the rest of the pressure values are determined by setting the permeability-ratios or value of lambda to 1.0 everywhere else on the grid.

The reservoir simulated in this study had two wells and the solution algorithm took this into account by determining the $\mathrm{x}, \mathrm{y}, \mathrm{z}$ distances, solid angles and radial distances from both wells to each grid point under consideration. The resulting numeric information was used to determine correct lambda values which could then be applied in Equation (8-20) to determine pressures. In general, when a reservoir has more than one well, all of the equations outlined in the previous description must be solved from the perspective of each well and the respective information combined in the calculation of the lambda coefficients.

For the calculations of this study, the value of the volumetric injection rate, Q, was set to 250 for the injector well and to -250 for the producer well. Any reasonable volumetric injection rate could have been used. At any time in the solution algorithm, if the value of the radial distance from the well to a given grid point becomes zero in any direction, the radius becomes that of the well for the calculation in that respective direction. The radius of the well is always scaled to be the ratio of a three inch radius (six inch diameter wellbore) divided by the length of one of the dimensions of a simulation grid block. For example, a well radius of 0.0025 , which was used by Weber, corresponds to a 3 inch radius well and grid blocks representing 100 feet on each side.

Around a given well, Weber calculated coefficients for only the interior cell faces of the $3 \times 3 \times 3$ cluster, a total of eighteen in each direction. This same constraint was 
placed on the coefficients as they were applied to the solution in this study. As the grid was refined, this constrained pattern was scaled and appropriately resized for the finer grids used.

\subsection{Summary}

The math of Weber et al. ${ }^{27}$ is summarized in this Chapter. The end result is six lambda (Weber) coefficients that can be easily incorporated into traditional finitedifference formulations to calculate accurate pressure solutions. Weber's coefficient need only be calculated for the interior cell faces of a block of cells around the well. For the coarsest grid considered, a block of twenty-seven cells was used which resulted in the calculation of eighteen coefficients in each direction. Finer grids required the calculation of more coefficients, as the same pattern was applied over a larger number of grid cells. 


\section{CHAPTER 9}

\section{STUDY WITH WEBER'S COEFFICIENTS APPLIED}

\subsection{Grid Geometries and General Setup}

The application of Weber's coefficients in the nested-grid method was conducted in a very similar manner to what was described in Chapter 6. A key difference in this portion of the study was that the coarse-grid points being nested into the finer grids actually came from a coarser grid. Previously the fixed points were applied in correct locations, but the fixed points were not actually obtained from smaller, coarser grids. To better validate the new method, Weber's equations were implemented in a practical manner, which meant that the coarse-grid points from a coarser grid size, had to be inserted into a more refined grid in physically the same location. Using Cartesian coordinates in three dimensions dictates that only certain grid sizes are capable of this refinement.

In the study using Weber's equations, a reservoir 500 feet by 500 feet by 1,000 feet was simulated. To ensure symmetry in the placement of the wells, the height and width of the reservoir must be represented by an odd number of grid points and the depth be represented by double the number of grid points allocated to the height or width. In this case, the smallest grid size that could be used to provide a reasonable representation of the reservoir was a 5x5x10 grid. To refine the grid in a way that ensured that calculated pressures from a 5x5x10 reservoir were honored by the new, 
more refined grid, required that the dimensions of the more refined grid be an odd multiple of the original. For example, in this portion of the study the larger, more refined grid sizes considered are odd multiples of the dimensions of the original course grid and are displayed in the following table.

Table V. Grid Dimensions and Size

\begin{tabular}{|c|c|}
\hline Grid Size & Total Grid Points \\
\hline 5X5X10 & 250 \\
\hline 15X15X30 & 6750 \\
\hline 25X25X50 & 31250 \\
\hline 35X35X70 & 85750 \\
\hline 45X45X90 & 182250 \\
\hline 55X55X110 & 332750 \\
\hline 65X65X130 & 549250 \\
\hline 75X75X150 & 843750 \\
\hline
\end{tabular}

Multiplying the coarse-grid dimensions by an odd number ensures that the courser grid-block pressure information can be properly placed in a more refined grid. This was essential in the passage of pressure information from coarser grids to finer grids. To illustrate this point, the following figures are included.

Figure 15 shows an end view of a $5 \times 5 \times 10$ reservoir. The numbers exterior to the grid represent overall height and width of the reservoir. The numbers on the interior of the cells represent the distance at the center of the particular grid cell in the respective $\mathrm{x}$, $\mathrm{y}$ and $\mathrm{z}$ directions. Simply put, the center of the first cell in Figure 15 is located fifty feet in the $\mathrm{x}, \mathrm{y}$ and $\mathrm{z}$ directions. In the nested-grid method, the calculated pressure value from this coarse grid is passed on to a finer grid where it becomes the pressure for the grid cell that is likewise centered at fifty feet by fifty feet by fifty feet. Figure 16 shows the more 
refined $15 \times 15 \times 30$ grid with the pressure solution locations of the $5 \times 5 \times 10$ grid highlighted within.

\begin{tabular}{|r|r|r|r|r|r|}
\cline { 2 - 6 } 100 & 50 & 150 & 250 & 350 & 450 \\
\hline 200 & 150 & & & & \\
\hline 300 & 250 & & & & \\
\hline 400 & 350 & & & & \\
\hline 500 & 450 & & & & \\
\hline
\end{tabular}

Figure 15. Coarse-Grid Dimensions in Feet

It is evident that the previously calculated pressures from the $5 \times 5 \times 10$ grid fit nicely into the $15 \times 15 \times 30$ grid and that the numerical value of the pressures calculated on the course grid can be maintained on the finer grid. This pattern of refinement can be continued on to extremely large grid sizes. As long as this same pattern of grid refinement is continued, one can start with a finer grid than $5 \times 5 \times 10$ as the original coarsegrid size and proceed in grid refinements from there. 


\begin{tabular}{|c|c|c|c|c|c|c|c|c|c|c|c|c|c|c|c|}
\hline 33.33 & $\frac{33.33}{16.7}$ & $\frac{66.67}{50}$ & $\frac{100.00}{83.3}$ & $\frac{133.33}{117}$ & $\frac{166.67}{150}$ & $\frac{200.00}{183}$ & $\frac{233.33}{217}$ & $\frac{266.67}{250}$ & $\frac{300.00}{283}$ & 317 & $\frac{366.67}{350}$ & $\frac{400.00}{383}$ & $\frac{433.33}{417}$ & $\frac{466.67}{450}$ & $\frac{500.00}{483}$ \\
\hline 66.67 & 50 & & & & & & & & & & & & & & \\
\hline 100.00 & 83.3 & & & & & & & & & & & & & & \\
\hline 133.33 & 117 & & & & & & & & & & & & & & \\
\hline 166.67 & 150 & & & & & & & & & & & & & & \\
\hline 200.00 & 183 & & & & & & & & & & & & & & \\
\hline 233.33 & 217 & & & & & & & & & & & & & & \\
\hline 266.67 & 250 & & & & & & & & & & & & & & \\
\hline 300.00 & 283 & & & & & & & & & & & & & & \\
\hline 333.33 & 317 & & & & & & & & & & & & & & \\
\hline 366.67 & 350 & & & & & & & & & & & & & & \\
\hline 400.00 & 383 & & & & & & & & & & & & & & \\
\hline 433.33 & 417 & & & & & & & & & & & & & & \\
\hline 466.67 & 450 & & & & & & & & & & & & & & \\
\hline 500.00 & 483 & & & & & & & & & & & & & & \\
\hline
\end{tabular}

Figure 16. 5x5x10 Coarse-Grid Pressures Nested Into a 15x15x30 Grid

To extend the grid sizes considered, this study included very coarse grids that were subsets of the $5 \times 5 \times 10$ grid. For example, the fewest number of coarse-grid points used was sixteen. These sixteen points were selected from the solution of a $5 \times 5 \times 10$ grid and nested into larger grids. An algorithm to do this was developed to ensure that the location and pressure information of the points being inserted into the finer grids was not compromised.

To fix any point taken from a coarse grid into a finer grid, the $\mathrm{x}, \mathrm{y}, \mathrm{z}$ coordinates of a given point on the coarse grid were taken and multiplied by the same multiple that 
the fine grid was of the course grid. For the grid sizes used in this study the multipliers were 1,3,5,7,9,11,13 and 15. Once multiplied by the specified multiplier, the corresponding spacing value was subtracted from this value. The spacing value was calculated by subtracting one from the multiplier and then dividing by two. In this study the spacing values were $0,1,2,3,4,5,6$ and 7 . For example, from a coarse grid of $5 \times 5 \times 10$, if we looked at the point 2,2,2, its corresponding coordinates on a finer grid of $15 \times 15 \times 30$ become 5,5,5.

$$
(2 \cdot 3)-[(3-1) / 2]=5
$$

This simple pattern could be extended to any of the nested-grid set ups and was used in programming the nested-grid method. Consequently, in the nested-grid study a particular pattern could be selected on the original coarse grid and then using the method described above, that same pattern could be maintained on successively finer and finer grids.

\subsection{Nested-Grid Results}

With Weber's equations and the method for proper transfer of pressure information during nested-grid calculations established, the computer algorithm developed in Fortran 90 generated results. Table A.XVII in Appendix A displays the results obtained. The table displays the fine-grid dimensions, the total number of fine-grid points, the total number of coarse grid points, the optimal over-relaxation factor, the number of iterations required for convergence, the time required for convergence, and the time per iteration. In this portion of the study the number of coarse-grid points includes 
the wells. Figure 17 shows the time required for convergence for the various nested-grid configurations considered.

Weber SOR: Time Required for Convergence

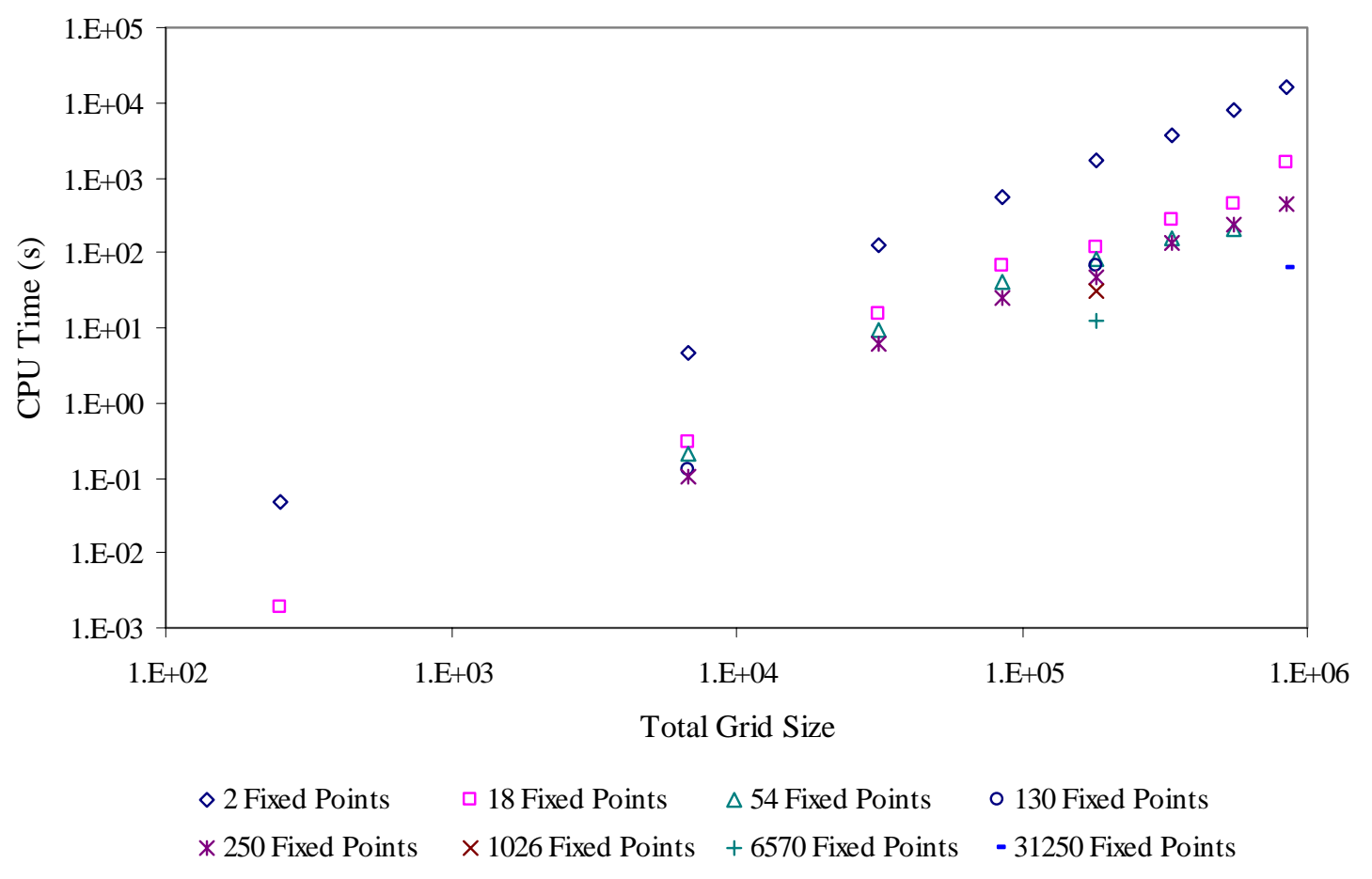

Figure 17. Weber SOR: Time Required for Convergence of Various Nested-Grid Configurations

\subsection{Regression and Dimensionless Time Analysis}

Regression of the performance data recorded in Table A.XVII was conducted and correlations for $\omega_{\text {opt }}$ and $\mathrm{N}_{\text {Iter }}$ were developed and applied in a dimensionless time analysis. These initial results of are found in Appendix C. After reviewing all of the data collected, it was determined that better mathematical correlations for $\omega_{\text {opt }}$ and $\mathrm{N}_{\text {Iter }}$ could be developed. The finalized regression and dimensionless time analysis are discussed in Chapters 10 and 11. 


\subsection{Summary}

The nested-grid study applying Weber's coefficients was conducted in a very similar manner to that discussed in Chapter 6. Fine-grid sizes that were odd multiples of the original coarse-grid size allowed for the accurate coarse-grid pressure solutions of Weber to be nested into desired fine grids. Various nested-grid configurations were considered and the resulting performance data was recorded (See Table A.XVII). 


\section{CHAPTER 10}

\section{REGRESSION OF ALL RESULTS}

Previous work, as recorded in Appendix B and C, indicated that mathematical correlations could be generated from performance data to predict the number of iterations required to reach convergence for any nested-grid setup as well as the optimal over-

relaxation factor. The regression derived correlations were essential to the optimization of the nested-grid algorithm and achieved the sixth objective of the study. These earlier correlations were reviewed and it was determined that better correlations could be made that would be more viable and potentially fit the data better. In summary, the data sets regressed were LaPlace SOR, GMRES, and Weber SOR.

\subsection{Regression of LaPlace and Weber SOR}

The performance data from the study done using LaPlace SOR and Weber SOR was combined and regressed as a whole. In order to fit the data that was generated by Successive-Over-Relaxation, the following logarithmic fit of the data was used. The logarithm of the number of iterations required for convergence was calculated and a correlation of the following form was generated to match the actual value.

$$
\mathrm{LOG}_{10}\left(\mathrm{~N}_{\mathrm{ITER}}\right)=\mathrm{A}+\mathrm{B} \cdot \mathrm{LOG}\left(\mathrm{N}_{\mathrm{CG}}{ }^{\prime}\right)+\mathrm{C} \cdot \mathrm{LOG}\left(\mathrm{N}_{\mathrm{FG}}{ }^{\circ}-\mathrm{N}_{\mathrm{CG}}{ }^{\prime}\right)
$$


This equation is equivalent to the following exponential equation.

$$
\mathrm{N}_{\mathrm{ITER}}=\mathrm{A} \cdot\left(\mathrm{N}_{\mathrm{CG}}{ }^{\prime}\right)^{\mathrm{B}} \cdot\left(\mathrm{N}_{\mathrm{FG}}{ }^{\prime}-\mathrm{N}_{\mathrm{CG}}{ }^{\prime}\right)^{\mathrm{C}}
$$

$\mathrm{A}, \mathrm{B}$ and $\mathrm{C}$ are constants determined by the linear regression and $\mathrm{N}_{\mathrm{CG}}$ ' and $\mathrm{N}_{\mathrm{FG}}$ ` are described as follows.

$$
\begin{aligned}
& \mathrm{N}_{\mathrm{CG}}{ }^{\prime}=\mathrm{N}_{\mathrm{CG}}-\mathrm{N} \\
& \mathrm{N}_{\mathrm{FG}}{ }^{`}=\mathrm{N}_{\mathrm{FG}}-\mathrm{N}
\end{aligned}
$$

In these expressions $\mathrm{N}_{\mathrm{CG}}$ is simply the number of course grid points nested into the fine grid and $\mathrm{N}_{\mathrm{FG}}$ is the total number of fine grid points. The quantity $\mathrm{N}_{\mathrm{FG}}{ }^{`}-\mathrm{N}_{\mathrm{CG}}{ }^{`}$ is the number of unknown grid points in the fine-grid solution. The parameter $\mathrm{N}$ was introduced to attempt to physically account for the fact that the wells in Weber's solution are not as effective in relaxing the solution as the other fixed points because of the low permeabilites around them. If $\mathrm{N}=0$ there is no reduction in effectiveness. If $\mathrm{N}=2$ the wells are totally ineffective. In the regression, the constraint $\mathrm{N} \leq 2$ was used. This equation was used to fit the data from LaPlace SOR and Weber SOR in one regression where a separate value of $\mathrm{N}$ was regressed from the Laplace data and a separate value of $\mathrm{N}$ was regressed from the Weber data. The values of $\mathrm{A}, \mathrm{B}$, and $\mathrm{C}$ were constant. The end result was a correlation that predicted the number of iterations required for convergence that could be adjusted to fit the data from Laplace SOR or Weber SOR simply by changing the value of $\mathrm{N}$. The correlation determined from the regression of the data is as follows. 


$$
\begin{gathered}
\operatorname{LOG}\left(\mathrm{N}_{\mathrm{ITER}}\right)=0.9460-0.3531 \cdot \mathrm{LOG}\left(\mathrm{N}_{\mathrm{CG}}{ }^{\prime}\right)+0.4127 \cdot \mathrm{LOG}\left(\mathrm{N}_{\mathrm{FG}}{ }^{\prime}-\mathrm{N}_{\mathrm{CG}}{ }^{\prime}\right) \\
\mathrm{N}_{\mathrm{LAPLACE}}=1.5373 \\
\mathrm{~N}_{\mathrm{WEBER}}=1.9915
\end{gathered}
$$

The correlation had an overall $\mathrm{R}^{2}$ value of 0.986 , with and $\mathrm{R}^{2}$ value of 0.985 for LaPlace SOR data and a $\mathrm{R}^{2}$ value of 0.990 for Weber SOR data. The following figure shows how the calculated and actual values compare for LaPlace SOR and Weber SOR. The $\mathrm{R}^{2}$ value on the plot is the overall value.

\section{LOG(Niter) Combined LaPlace/Weber SOR}

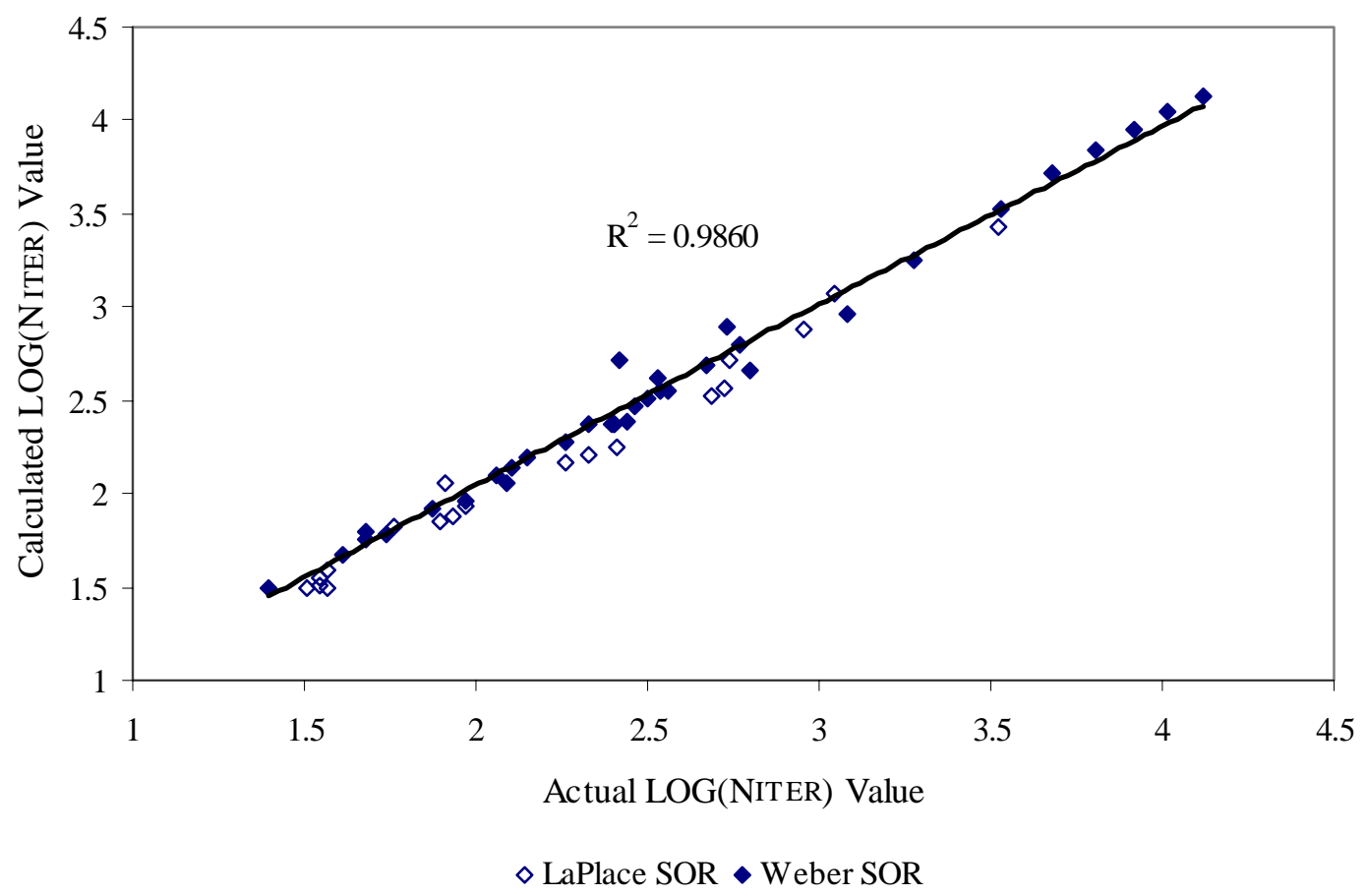

Figure 18. LaPlace and Weber SOR Combined Data Set Regression

Another important variable that needed to be predicted from the data collected was the optimal over-relaxation factor for any nested-grid configuration. This correlation 
was also generated by using all of the data from LaPlace SOR and Weber SOR. The general expression is as follows

$$
\omega_{\text {opt }}=2-\left[\mathrm{A} \cdot\left(\mathrm{N}_{\mathrm{CG}}{ }^{\circ}\right)^{\mathrm{B}} \cdot\left(\mathrm{N}_{\mathrm{FG}}{ }^{`}-\mathrm{N}_{\mathrm{CG}}\right)^{\mathrm{C}}\right]
$$

$\mathrm{A}, \mathrm{B}$, and $\mathrm{C}$ were determined by the regression as well as values of $\mathrm{N}$ for both LaPlace and Weber data. The expression, with the value of the variables determined from the regression, is as follows.

$$
\begin{gathered}
\omega_{\text {opt }}=2-\left[1.6923 \cdot\left(\mathrm{N}_{\mathrm{CG}}{ }^{\circ}\right)^{0.4310} \cdot\left(\mathrm{N}_{\mathrm{FG}}{ }^{\circ}-\mathrm{N}_{\mathrm{CG}}{ }^{\prime}\right)^{-.4464}\right] \\
\mathrm{N}_{\text {LAPLACE }}=0.0000 \\
\mathrm{~N}_{\text {WEBER }}=1.9922
\end{gathered}
$$

This resulted in an overall $\mathrm{R}^{2}$ value of 0.995 , with an $\mathrm{R}^{2}$ value of 0.998 and 0.990 for the LaPlace and Weber data respectively. The following figure show the how well the calculated and actual ORF values agree for LaPlace SOR and Weber SOR; the $\mathrm{R}^{2}$ value shown on the plot is the overall value. Expression (10-7) can be used to predict the optimal over-relaxation factor for any nested-grid configuration of the idealized reservoirs studied. The beginnings of a program that determines the optimal overrelaxation factor as part of the solution algorithm of Successive-Over-Relaxation for any grid is reported in Appendix D. 
Combined LaPlace/Weber SOR Over-Relaxation Factor (ORF)

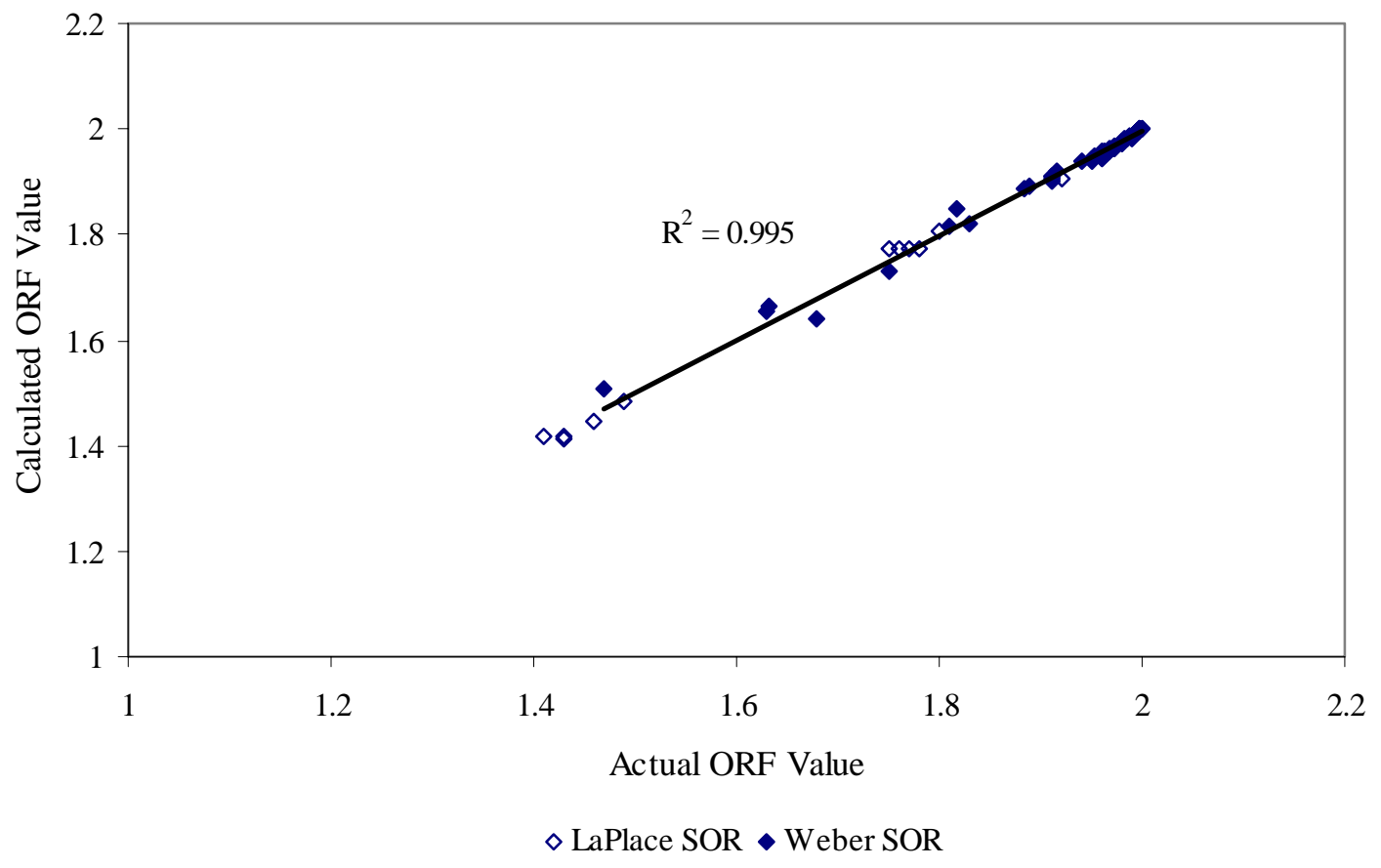

Figure 19. LaPlace and Weber SOR Combined ORF Regression

\subsection{GMRES Regression}

The general form of the correlation used to fit the data from GMRES to determine the number of iterations required for convergence took the following form.

$$
\mathrm{N}_{\text {ITER }}=\mathrm{A} \cdot\left(\mathrm{N}_{\mathrm{FG}}-\mathrm{N}_{\mathrm{CG}}\right)^{\mathrm{B}} \cdot\left(1+\mathrm{C} \cdot \mathrm{N}_{\mathrm{CG}}\right)^{\mathrm{D}}
$$

A, B, C, and D are the variables determined by the regression. The regression generated the various fixed parameters which are shown in the following correlation.

$$
\mathrm{N}_{\mathrm{ITER}}=2.8952 \cdot\left(\mathrm{N}_{\mathrm{FG}}-\mathrm{N}_{\mathrm{CG}}\right)^{0.3593} \cdot\left(1+0.0031 \cdot \mathrm{N}_{\mathrm{CG}}\right)^{-0.4577}
$$


The correlation had an $\mathrm{R}^{2}$ value of 0.995 . GRMES does not require an optimal overrelaxation factor.

NITER GMRES

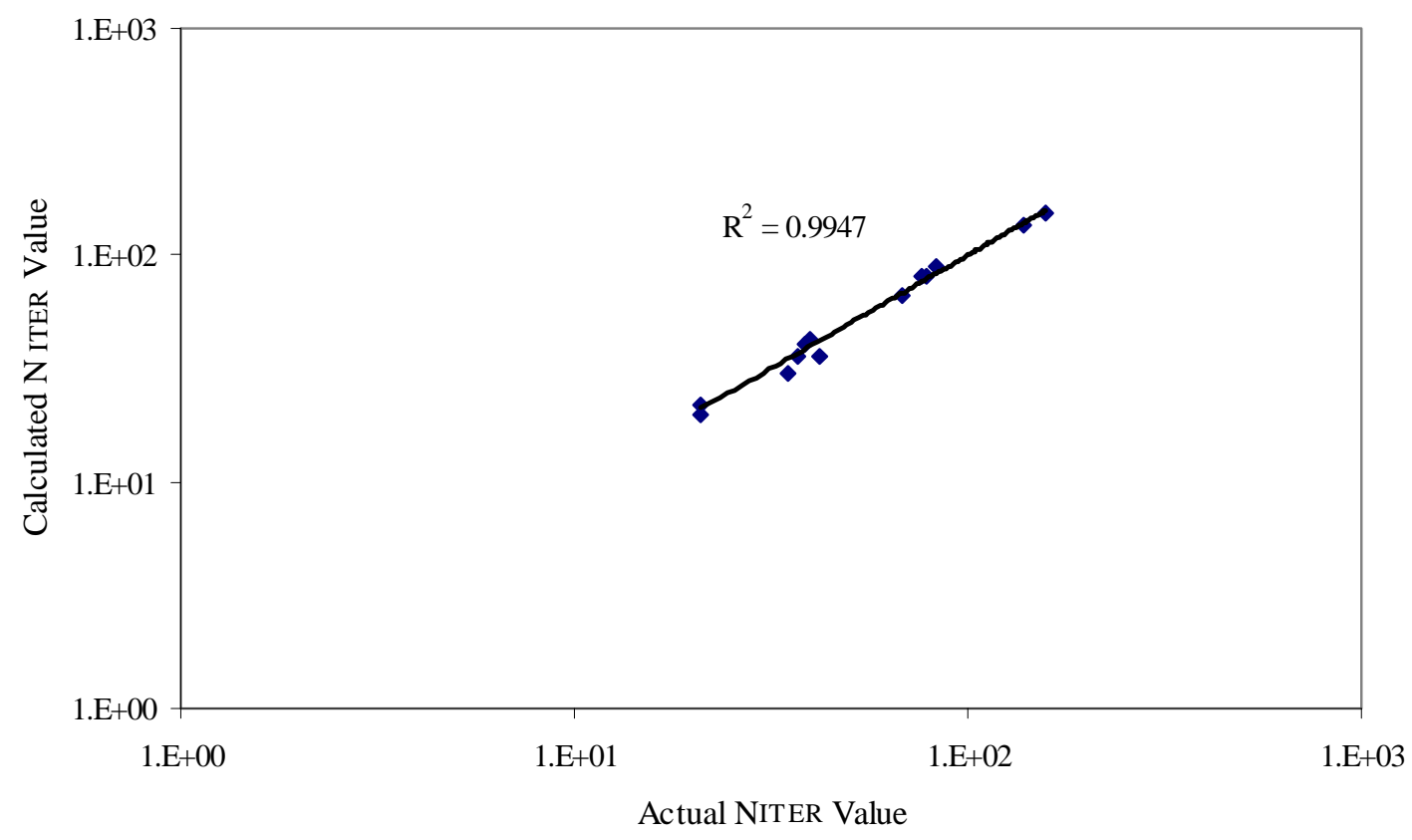

Figure 20. GMRES Regression

\subsection{Summary}

The performance data from LaPlace SOR, Weber SOR, and GMRES were regressed to generate mathematical correlations that can be used to predict the number of iterations required for convergence as well as the optimal over-relaxation factor for any nested-grid configuration. The correlations predicted the actual values of number of iterations and over-relaxation factor very well. These correlations will be used in the dimensionless time analysis of the next chapter. 


\section{CHAPTER 11}

\section{DIMENSIONLESS CPU TIME AND OPTIMUM COARSE GRID SIZE}

\subsection{Dimensionless Time}

Previous simulation runs for various grid sizes at different levels of coarse-grid refinement (Chapters 6 and 9) identified optimal performance of the nested-grid method for the three solution algorithms considered. A plot of the ratio of time/iteration for all of the nested-grid setups as a function of the total grid size is shown in Figure 21 for all of the data sets.

Time/Iteration vs Total Grid Size

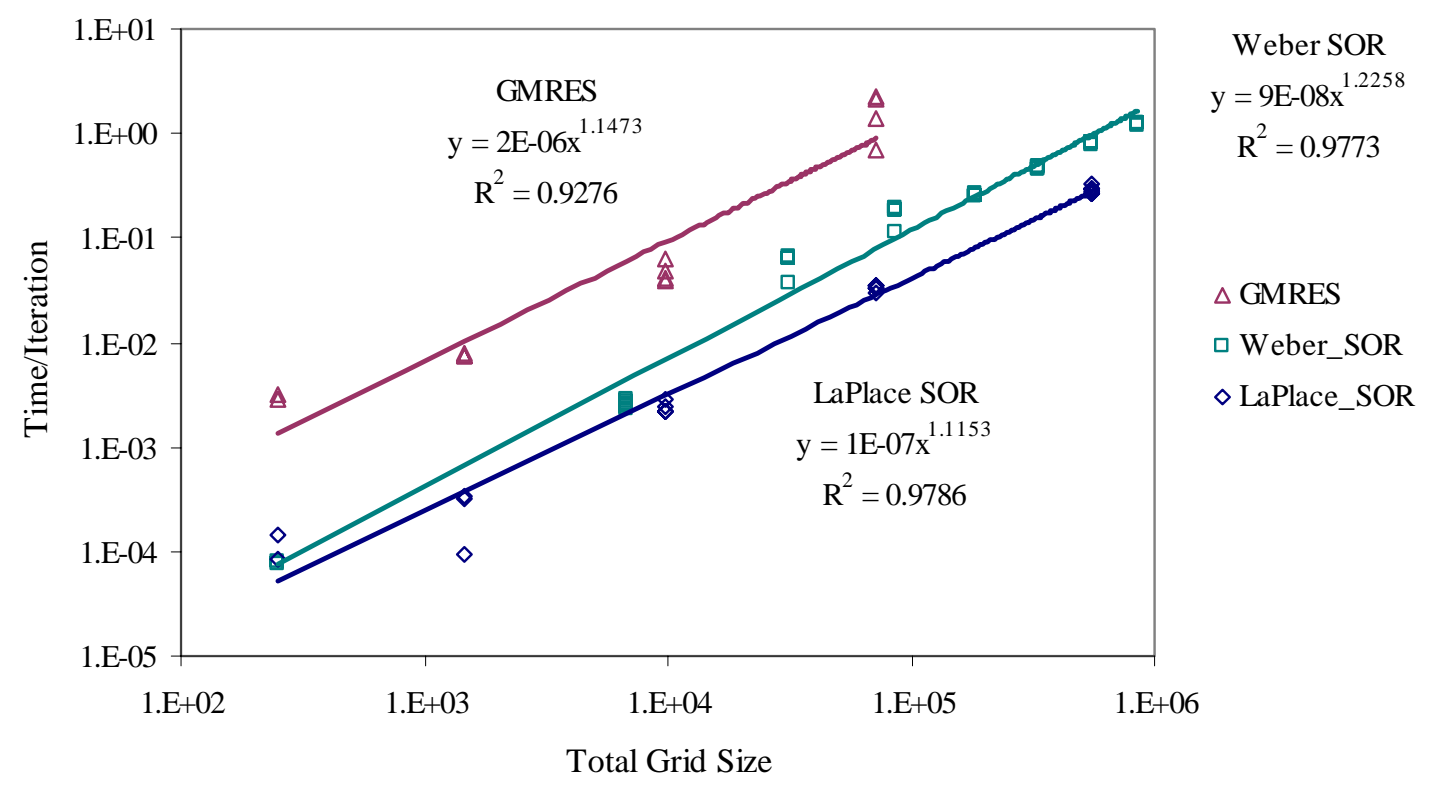

Figure 21. Time/Iteration as a Function of Total Grid Size 
The time/iteration was found to vary more or less directly with the total number of fine-grid points for all data sets. This observation suggested that the number of iterations multiplied by the grid size might serve as a dimensionless time value which was independent of the computer being used. The dimensionless cpu time expression was in turn used to determine the optimal number of fixed-coarse-grid points to be nested into a given fine grid, achieving another overall objective of the study. The expression for the total dimensionless time was.

$$
\mathrm{t}_{\mathrm{d}}=\mathrm{N}_{\mathrm{CG}} \cdot \mathrm{N}_{\mathrm{ITER}}\left(2, \mathrm{~N}_{\mathrm{CG}}\right)+\mathrm{N}_{\mathrm{FG}} \cdot \mathrm{N}_{\mathrm{ITER}}\left(\mathrm{N}_{\mathrm{CG}}, \mathrm{N}_{\mathrm{FG}}\right)
$$

As evident, the expression is made of up two terms: the time required for the calculation of the coarse grid with two wells, and the time required for the calculation of the nested-grid solution with two wells. The number of iterations, $\mathrm{N}_{\text {ITER }}$, is a function of the number of nested-coarse-grid points and the total number of fine-grid points $\mathrm{N}_{\mathrm{ITER}}\left(\mathrm{N}_{\mathrm{CG}}, \mathrm{N}_{\mathrm{FG}}\right)$ as discussed in Chapter 10. For the first term of expression (11-1), which determines the coarse-grid-dimensionless time, the number of iterations is a function of the number of wells (which become nested-coarse-grid points) and the number of coarsegrid points (which become equivalent to the total number of fine-grid points in this case). The second term of expression (11-1) is determined as specified with no special significance of the variables involved. The dimensionless time required for the calculation of either the coarse or fine-grid pressure solution is the product of the number of coarse or fine grid points used to model the reservoir with the number of iterations required for convergence of the respective coarse or fine grid. The sum of these two 
terms yields total dimensionless time. The function value for dimensionless time was minimized by determining the optimum number of fixed-coarse-grid points that should be nested in a given fine grid. The optimization was accomplished using the Solver tool in Microsoft Excel and the results recorded for Laplace SOR, Weber SOR, and GMRES.

\subsection{LaPlace SOR: Optimization of the Nested-Grid Method}

Figure 22 shows a plot of the improvement obtained for the LaPlace SOR method by the optimized-nested-grid method. The line, 2 Fixed Points, represents the dimensionless time required to obtain the fine-grid pressure solution with only the wells acting as fixed points, and the line, Optimal Number of Fixed Points, represents the dimensionless time required to obtain the fine-grid pressure solution using the optimal number of nested-grid pressures.

\section{Dimensionless Time: LaPlace SOR}

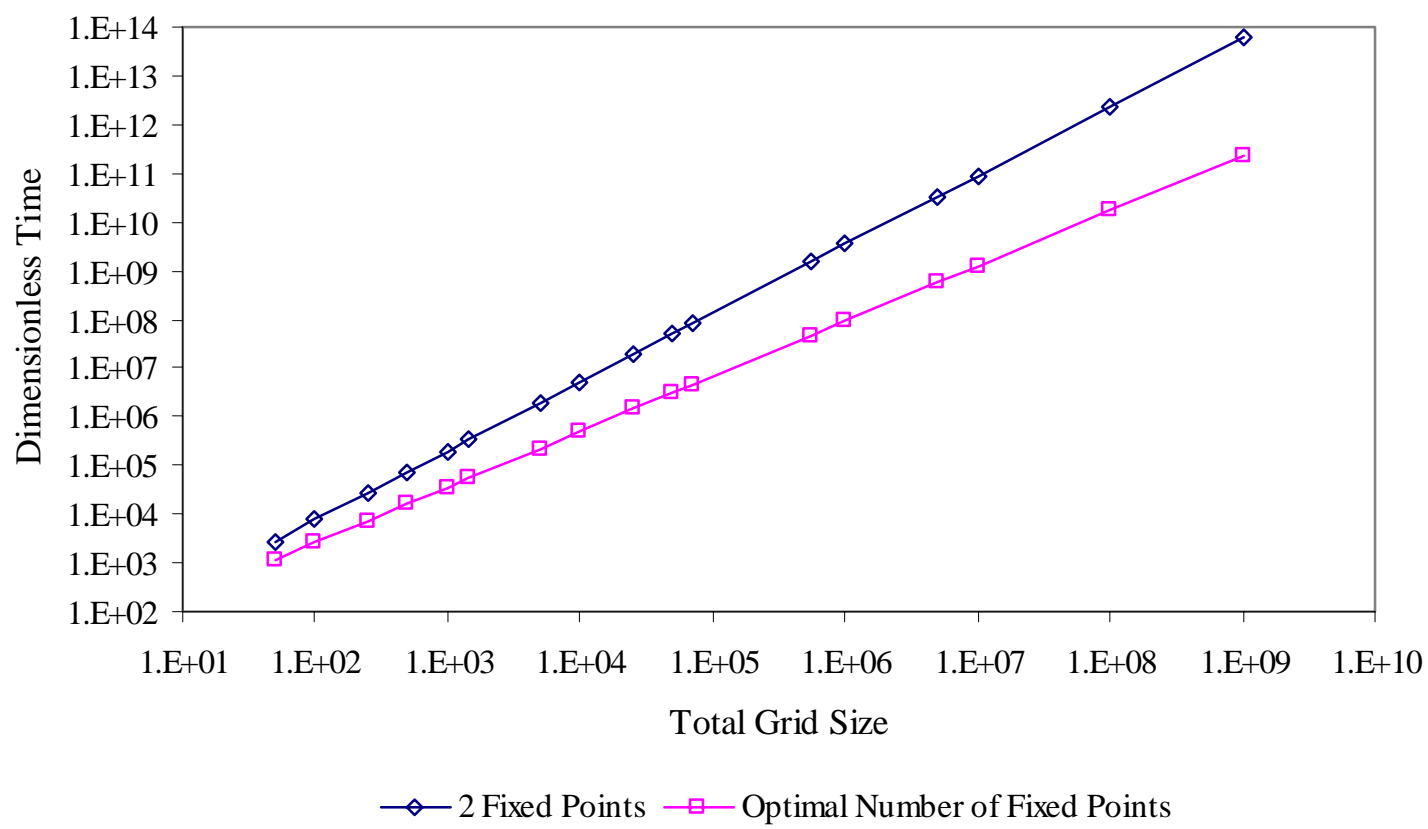

Figure 22. Laplace SOR: Improvement Obtained by the Nested-Grid Method 
Table A.XVIII summarizes the data displayed in Figure 22 and shows the ratio of improvement obtained by using the optimized-nested-grid method. For a grid size of one million, an improvement of 38 times over the tradition solution method was noted. Extrapolating the data to one billion grid points yielded an improvement of 263 times. This assumed that the computer being used could handle such large memory demands; the desktop used in this study could not.

For these nested-grid set ups, it was determined that at the optimal number of fixed-coarse-grid points, the calculation of the coarse-grid pressures took about $21 \%$ of the total time on average, with the nested-fine-grid calculation taking the remainder of the time. It was clear that for LaPlace SOR the nested-grid method reduced calculation time significantly; the ratio of improvement varied a value of 2 on the coarest grid to a value of 263 on the finest grid.

\subsection{GMRES: Optimization of the Nested-Grid Method}

A similar dimensionless time study was done to compare the performance of GMRES with GMRES using the nested-grid method. The previous Figure 21 shows a plot of the time/iteration as a function of grid size. The time/iteration was found to vary more or less directly with the total number of fine-grid points. A dimensionless time analysis was conducted using Equation (11-1) multiplied by twenty to correct the variation in constants determined from the power-law fit shown in Figure 21. This was done so that the three algorithms considered could eventually be compared directly. The results of the GMRES study are shown in the following figure. 


\section{Dimensionless Time: GMRES}

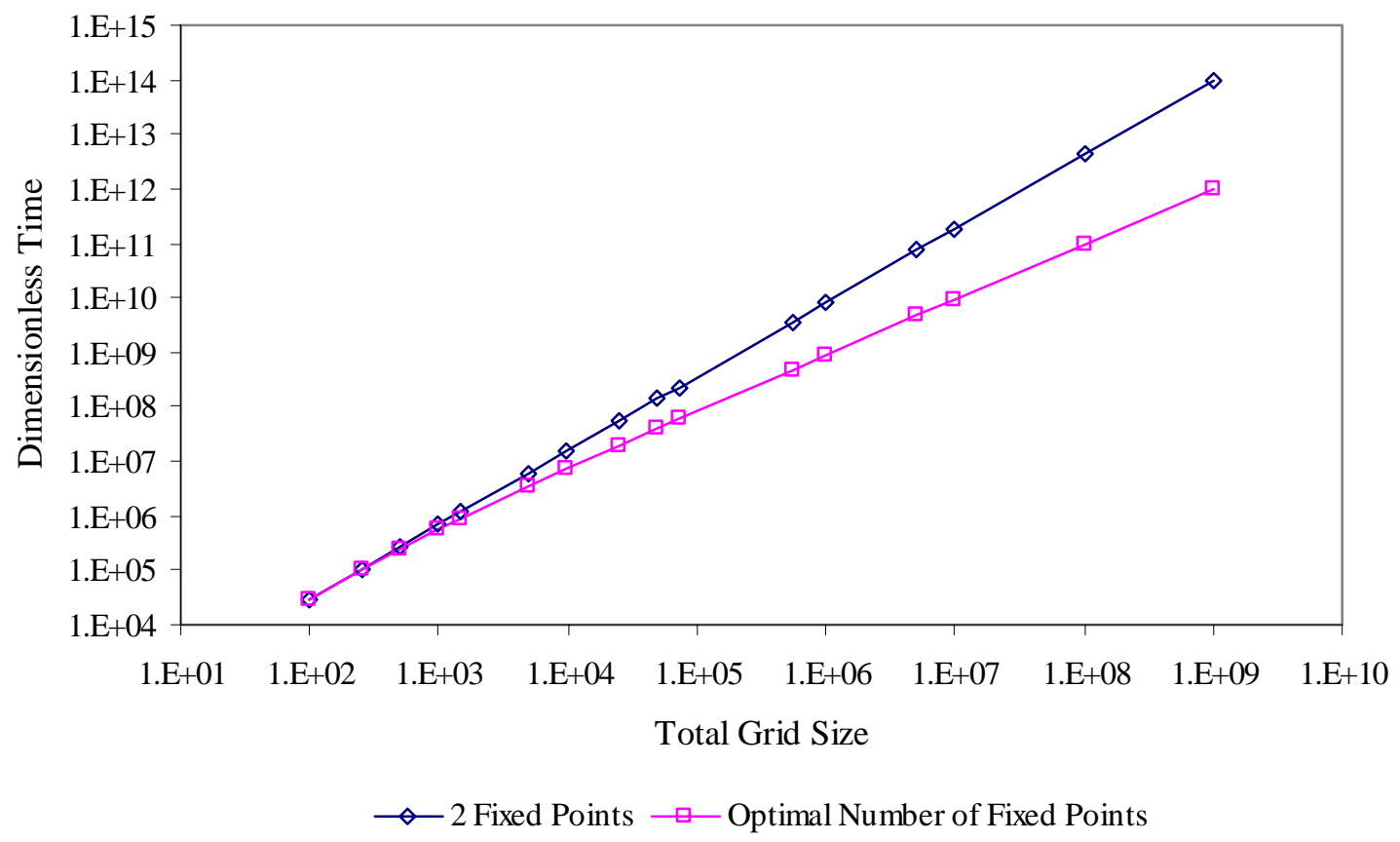

Figure 23. GMRES: Improvement Obtained by the Nested-Grid Method

Figure 23 indicates that GMRES also benefits from the implementation of the nested-grid method. At a grid size of one million the ratio of improvement is 9 times and at a grid size of one billion the ratio is 95. Table A.XIX in Appendix A tabulates the results shown in Figure 23. On average the coarse-grid calculation took about $26 \%$ of the total dimensionless time.

\subsection{Weber SOR: Optimization of the Nested-Grid Method}

The dimensionless time results obtained using Weber's equations in the algorithm are shown in Figure 24 and are tabulated in Table A.XX. 


\section{Dimensionless Time: Weber SOR}

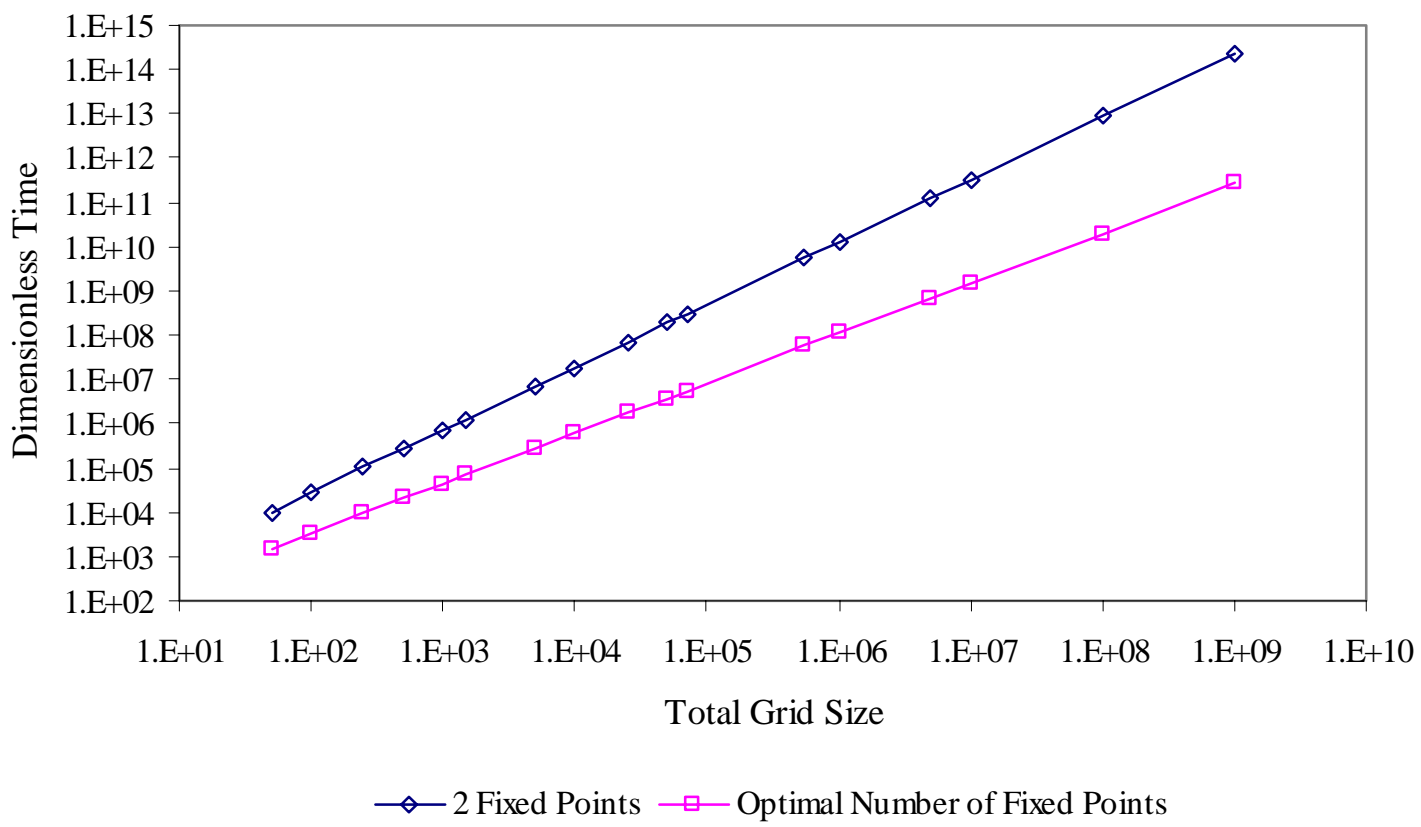

Figure 24. Weber SOR: Improvement Obtained by the Nested-Grid Method

These results indicate a significant increase in performance. At a grid size of one million, an improvement by a factor of 116 is noted, and at a grid size of one billion, an improvement of 813 is noted. For Weber SOR, the dimensionless time analysis was conducted using Equation (11-1) multiplied by 0.9 to correct the variation in constants determined by the power-law fit displayed in Figure 21. The coarse-grid solution took on average about $21 \%$ of the total dimensionless time required to obtain the finely-gridded pressure solution.

\subsection{Comparison of the Performance of the Different Methods}

A plot of the improvement ratio verses total grid size for the three methods is shown in the following figure. This figure indicates that Weber SOR showed the greatest 
ratio of improvement from the solution with two fixed points to the solution with the optimum number of fixed points nested into the fine grid.

\section{Ratio of Improvement}

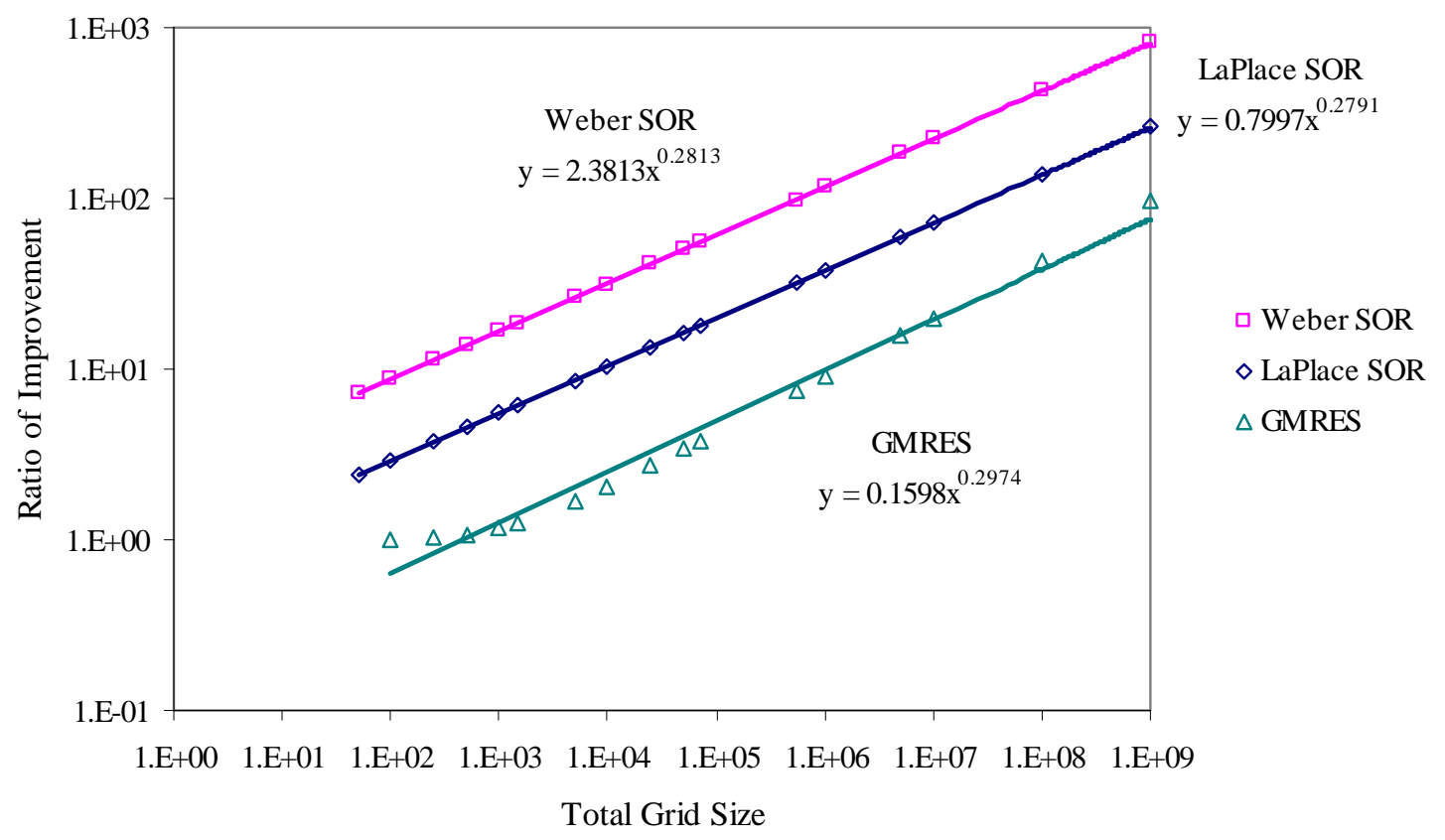

Figure 25. Ratio of Improvement

The next figure indicates how the algorithms LaPlace SOR, GMRES, and Weber SOR performed with respect to each other for their traditional and optimized solutions. Improved performance of these algorithms from their performance when only two wells were fixed was consistently observed. It is interesting to note that the SOR algorithm at its $\omega_{\text {opt }}$ performed better than even an advanced algorithm such as GMRES. These results indicate that the previous conclusion made in Chapter 4, that GMRES would not outperform LaPlace SOR at large grid sizes, was correct. Also of interest is the observation that despite different times required to generate a solution for LaPlace and Weber SOR when the only fixed points were the wells, at the optimal number of nested- 
grid points, their respective dimensionless times became very similar. This indicates that although generating coarse grids using Weber's math requires more time, when an optimal number of these coarse-grid pressure points are nested into a fine-grid, similar performance is seen as that for the optimal performance of LaPlace SOR. This shows that the cost of the time required to generate Weber's accurate solutions does not decrease its optimized performance.

Dimensionless CPU Time: A Comparison of All Algorithms

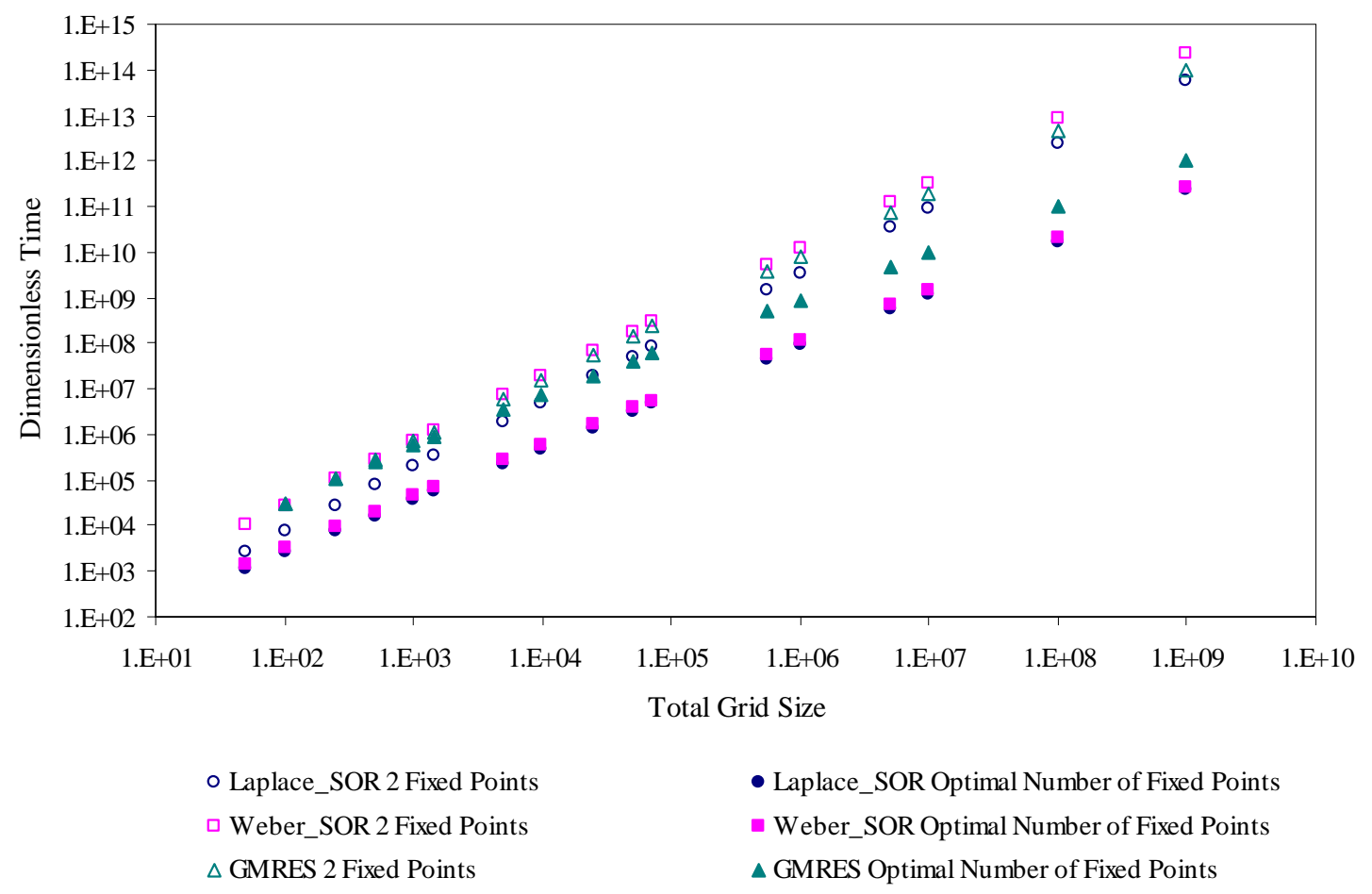

Figure 26. Dimensionless Time Summary

\subsection{Optimal Number of Fixed Points}

The dimensionless time analysis indicates an optimal number of fixed points to be nested into a given fine grid. Figure 27 shows how the optimal number of fixed points 
compares for each of the algorithms used to solve the problem. Weber SOR requires the least number of coarse-grid points to be nested into the fine-grid to obtain optimal performance. This is most likely due to the fact that the Weber's accurate coarse-grid solutions require the most time to generate a pressure solution.

\section{Optimal Number of Fixed Points vs Total Grid Size}

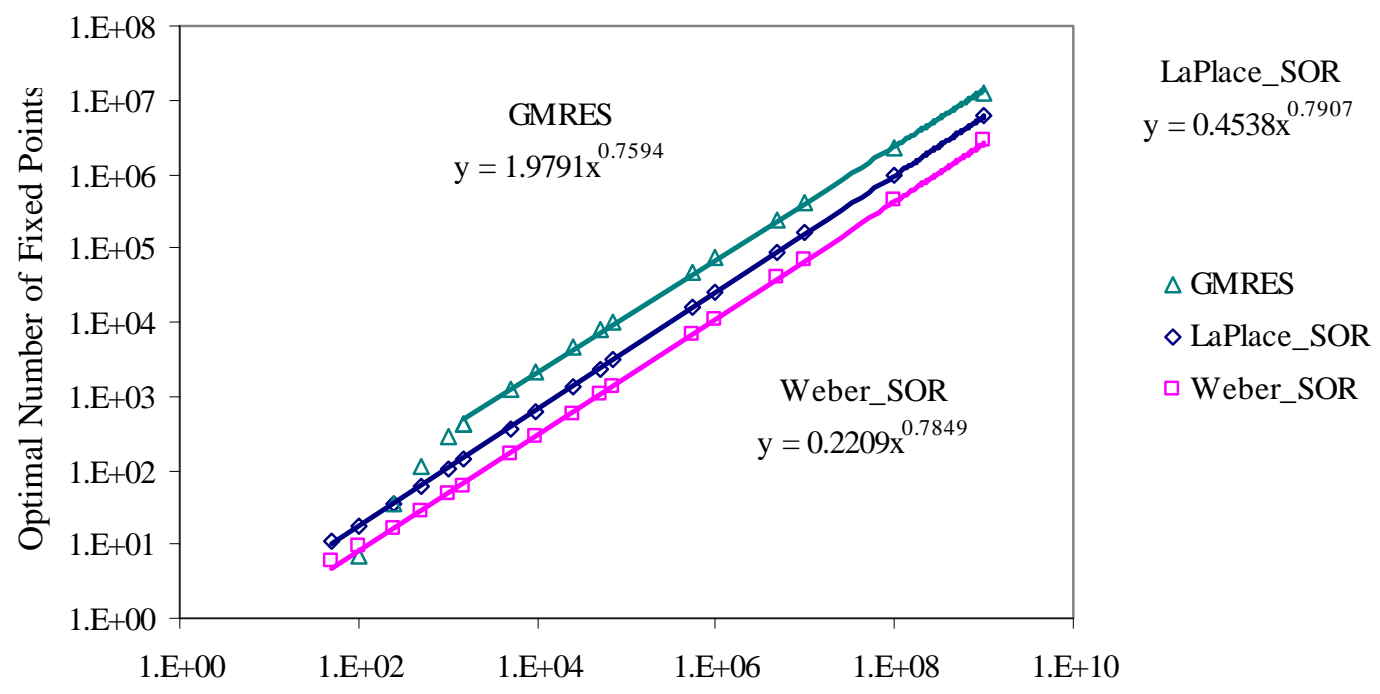

Total Grid Size

Figure 27. Optimal Number of Fixed Points

\subsection{Summary}

For LaPlace SOR, GMRES, and Weber SOR the time/iteration, determined from the performance data, was observed to vary more or less directly with total grid size. This allowed for a dimensionless cpu time expression to be developed which incorporated the mathematical correlations described in Chapter 10 and represented the actual cpu time required for convergence of a particular nested-grid configuration. The dimensionless time expression was minimized by optimizing the number of coarse-grid points in any 
given nested-grid setup. The results showed significant reductions in the amount of computational time required to obtain finely-gridded pressures solutions. Weber SOR ratio of improvement ranged from 7 on the coarsest nested-grid setup to 813 on the finest nested-grid. This verified that the solution method developed in this thesis generates fast, accurate pressure solutions on fine grids. 


\section{CHAPTER 12}

\section{ERROR ANALYSIS}

To determine the accuracy of the solution obtained using the nested-grid method a one-step, fine-grid solution was generated for each of the grid sizes in the nested-grid study using the algorithm that applied Weber's Equations. A rigorous convergence criterion of $10^{-9}$ was set. For these solutions, there were no fixed points embedded in the desired solution grid which meant that the solution simply relaxed around the values of the two wells until the convergence criterion was satisfied. These pressure solutions were generated to obtain an accurate pressure solution for the given reservoir at different grid sizes against which the pressures generated from the nested-grid method at the same grid size could be compared. Weber's previous work showed that his equations made the solution four orders of magnitude more accurate than traditional finite-difference formulations and very close to the analytical solution. The data displayed in Figure 1 of Chapter 2 showed that for Weber's inverse-r finite volume method the average error (psi) for an 11x11x22 grid from the analytical solution was 0.0182 and the maximum error was 0.0757 . The single-grid solutions generated in this error analysis contained this four order of magnitude improvement in accuracy.

With the pressure solutions obtained for various grid sizes, the pressures from the nested-grid study, which utilized Weber’s equations, were calculated. The convergence criterion was set to $10^{-6}$. The nested-grid solutions were then compared with the 
previously obtained single-step solutions. The comparison of these two solutions was completed through two calculations. First, the absolute difference between the two solutions was determined as expressed in the following equation.

$$
\text { Absolute Difference = ABS [(One-Step Solution })-(\text { Nested-Grid Solution })]
$$

The second calculation communicated the percent difference in the nested-grid solution from the original single-step solution obtained at the convergence criterion of $10^{-9}$. The following equation expresses the calculation of this value.

\author{
Absolute Percentage Difference from One-Step Solution = \\ [(Absolute Difference)/(One-Step Solution) ${ }^{*} 100$
}

\title{
12.1 Error Analysis Results
}

The values of these two calculations were tabulated in Excel and the average and maximum values for each grid were determined and recorded in the following tables. For grid sizes larger than $25 \times 25 \times 50$ only half of the reservoir was analyzed to make the analysis more manageable in Excel. The symmetry of the pressure solution permitted this. For the tables referred to in this chapter, the number of fixed points includes the wells. For Tables A.XXI and A.XXII, when the number of fixed points is 18, 54 or 250 the fixed points were taken from a 5x5x10 grid. The maximum and average absolute difference results are displayed in Table A.XXI of Appendix A. The absolute values show that the maximum absolute difference for the grids considered never exceeds 
0.1902 and the average absolute difference never exceeds 0.0168 . These errors are of the same magnitude of found by Weber (0.0757 maximum and 0.0182 average) and hence demonstrate the nested-grid solution method does not substantially decrease the extraordinary accuracy of his solution. The well pressures are at \pm 1500 psi, hence these maximum and average differences correspond to only $0.0067 \%$ and $0.0006 \%$ of the overall pressure drop, respectively. In all cases, the maximum error on the nested-grid solution occurred at fixed grid points, i.e. at the location of the nested-grid points that came from a coarser grid. The coarsest grid used to generate nested-grid points was the $5 \times 5 \times 10$ grid.

Table A.XXII records the maximum and average percent difference for the same data. It is evident from this table of nested-grids that all maximum percent differences recorded were under 5\%. The nested-grid solution varies from the single-step solution, that is already four orders of magnitude more accurate, by under $5 \%$ at most and on average by less than $1 \%$. This meant that all points generated using the nested-grid method were at least 95\% accurate with respect to the original single-step solution.

The coarsest grid (5x5x10) used to generate nested-grid points in Table A.XXII introduced less than 5\% error around the coarse-grid points that were fixed into the desired fine-grid. If a finer grid, such as a $15 \times 15 \times 30$ or a $25 \times 25 \times 50$, was used to generate coarse-grid points, the maximum and average error was reduced. The following two tables show how the maximum and average absolute difference were reduced significantly when 18,54 , and 250 coarse-grid points were nested into a 25x25x50 fine grid that had coarse-grid points coming from a 15x15x30 grid instead of a 5x5x10 grid. 
Table VI. Nested-Grid Points Take From a 5x5×10 Grid

\begin{tabular}{|c|c|c|c|}
\hline Grid Dimensions & $\begin{array}{c}\text { Number of } \\
\text { Fixed Points }\end{array}$ & $\begin{array}{c}\text { Maximum } \\
\text { Absolute } \\
\text { Difference }\end{array}$ & $\begin{array}{c}\text { Average } \\
\text { Absolute } \\
\text { Difference }\end{array}$ \\
\hline $25 X 25 X 50$ & 18 & 0.0411 & 0.0104 \\
\hline $25 X 25 X 50$ & 54 & 0.1489 & 0.0156 \\
\hline $25 X 25 X 50$ & 250 & 0.1901 & 0.0065 \\
\hline
\end{tabular}

Table VII. Nested-Grid Points Taken From a 15x15x30 Grid

\begin{tabular}{|c|c|c|c|}
\hline Grid Dimensions & $\begin{array}{c}\text { Number of } \\
\text { Fixed Points }\end{array}$ & $\begin{array}{c}\text { Maximum } \\
\text { Absolute } \\
\text { Difference }\end{array}$ & $\begin{array}{c}\text { Average } \\
\text { Absolute } \\
\text { Difference }\end{array}$ \\
\hline 25 25X50 & 18 & 0.0009 & 0.0009 \\
\hline $25 X 25 X 50$ & 54 & 0.0160 & 0.0030 \\
\hline $25 X 25 X 50$ & 250 & 0.0160 & 0.0021 \\
\hline
\end{tabular}

From these two tables, it was clear that by using a finer grid to generate the coarse-grid points the absolute error was reduced. The next two tables show the maximum and average percent difference for the same arrangement.

Table VIII. Nested-Grid Points Taken From a 5x5x10 Grid

\begin{tabular}{|c|c|c|c|}
\hline Grid Dimensions & $\begin{array}{c}\text { Number of } \\
\text { Fixed Points }\end{array}$ & $\begin{array}{c}\text { Maximum \% } \\
\text { Difference }\end{array}$ & $\begin{array}{c}\text { Average \% } \\
\text { Difference }\end{array}$ \\
\hline 25X25X50 & 18 & 0.82 & 0.29 \\
\hline 25X25X50 & 54 & 3.65 & 0.46 \\
\hline 25X25X50 & 250 & 4.41 & 0.19 \\
\hline
\end{tabular}

Table IX. Nested-Grid Points Taken From a 15x15x30 Grid

\begin{tabular}{|c|c|c|c|}
\hline Grid Dimensions & $\begin{array}{c}\text { Number of } \\
\text { Fixed Points }\end{array}$ & $\begin{array}{c}\text { Maximum \% } \\
\text { Difference }\end{array}$ & $\begin{array}{c}\text { Average \% } \\
\text { Difference }\end{array}$ \\
\hline 25X25X50 & 18 & 0.03 & 0.03 \\
\hline 25X25X50 & 54 & 0.39 & 0.09 \\
\hline 25X25X50 & 250 & 0.50 & 0.05 \\
\hline
\end{tabular}


Once again, the fixed grids of 18 and 54 were sub-grids of the coarse $5 \times 5 \times 10$ grid and the 18, 54, and 250 fixed grids were sub-grids of the 15x15x30 coarse grid. From the last two tables, it is clear that the maximum and average percent difference is also reduced significantly by taking the nested-grid points from a finer grid, 15x15x30 instead of 5x5x10. The maximum percent difference in Tables VIII and IX was reduced by a factor of 27, 9 and 9 for the 18, 54, and 250 fixed points respectively. From Table A.XXII, the maximum percent difference from the $10^{-9}$ solution was less then $5 \%$ for any grid point in the fine-grid pressure solution when the coarsest grid, $5 \times 5 \times 10$, was used to generate the fixed points nested in the solution. A finer coarse grid, 15x15x30, decreases the maximum percent difference greatly. The trend of reduced percent difference as a finer coarse grid is used should hold throughout all possible nested-grid systems. It should be noted that for a given grid from which coarse-grid points are taken, if more fixed points are nested into a finer grid from that solution the result is more error in the final finely-gridded solution, as the error inherent in each fixed point influences the final solution.

It is true that Weber's solution is very accurate and we can probably assume that it is the actual analytical solution for the error analysis; however, the 5\% maximum error relative to the actual solution obtained using the coarsest grid in the study may still be disconcerting. A potential source of trouble with this analysis was that well pressures, \pm 1500 , which are physically unrealistic, were chosen, to get symmetric reservoir pressures. A zero pressure surface exists in the solution. Division by these zero's and near-zeros to get the percent errors may create misleading values. Percent difference would possibly look better if the well pressures were realistic values, for example 8,000 psi for the 
injection well and $-5,000$ psi for the production well. Also, a more rigorous convergence criterion than that describe in Chapter 3, would most likely reduce all measures of error.

\subsection{Summary}

Two measurements, absolute difference and absolute percentage difference from a one-step solution, were made to determine the amount of error inherent in the nested-grid method applied with Weber's equations. The absolute difference values showed that for the grid sizes considered, the maximum absolute difference never exceeds 0.1902 and the average absolute difference never exceeds 0.0168 . These errors are of the same magnitude found by Weber (0.0757 maximum and 0.0182 average) and demonstrate that the nested-grid solution method does not substantially decrease the extraordinary accuracy of his solution. For the coarsest grid used, the maximum percent difference recorded was under 5\%. This may be disconcerting, but potential remedies for this would be using a finer coarse grid, using more realistic well pressures that do not generate a symmetric solution or using a more rigorous convergence criterion. 


\section{CHAPTER 13}

\section{MULTIPLE NESTED-GRIDS}

In an effort to improve on the methods developed to this point and meet the last objective of this study, the idea of solving multiple grids to obtain the final finely-gridded pressure solution was considered and evaluated. It was anticipated that multiple nestedgrid systems might further reduce the amount of time required to obtain the final fine-grid solution.

The general idea behind this analysis was straight forward and involved using the dimensionless time analysis to determine how long multiple nested-grids systems took to determine finely-gridded pressures. To this point in the research, the optimal dimensionless time to obtain the full finely-gridded pressure solution was obtained by the following expression.

$$
t_{\text {total }}=t_{\text {coarse-grid }}+t_{\text {nested-grid }}
$$

This equation was replaced in this part of the study by the following expression.

$$
\mathrm{t}_{\text {total }}=\mathrm{t}_{\text {coarse-grid }}+\mathrm{t}_{1 \text { st nested-grid }}+\mathrm{t}_{2 \text { nd nested-grid }}+\mathrm{t}_{3 \text { rd nested-grid }}+\mathrm{t}_{4 \text { th nested-grid }}+\ldots
$$


As the expression suggests, there is a possibility that a large number of nested grids could be used in the calculation of overall dimensionless time required to obtain the full finegrid pressure solution. The analysis of dimensionless time was set up in Excel using the Solver tool to run an optimization analysis. The optimization process determined the appropriate size of intermediate nested grids for total grid sizes of $10^{3}, 10^{5}$, and $10^{6}$. This initial study was done to demonstrate in theory the potential of this process.

\subsection{Results}

The study was initially conducted for a total grid size of $10^{5}$. To help understand some of the logic of the optimization setup, the following tables are included which depict what a three nested-grid system involves. The ultimate goal of the optimization routine is to minimize the total time required to generate the full fine-grid solution. In this example, the sum of the dimensionless time required for the solution of the original coarse grid and three nested-grid systems was required to obtain the final solution. Table X shows the nested-grid systems generated by Excel's optimization software when three nested grids were used to determine the solution of the finely-gridded pressures for a grid size of $10^{5}$.

Table X. Size of Coarse and Fine Grids in Nested-Grid Method

\begin{tabular}{|c|c|}
\hline \multicolumn{2}{|c|}{ Three Nested Grids } \\
\hline $1^{\text {st }}$ Fixed Grid Size $\left(\mathrm{N}_{\mathrm{CG}}\right)$ & 169.3 \\
\hline $1^{\text {st }}$ Total Grid Size $\left(\mathrm{N}_{\mathrm{FG}}\right)$ & 5194.0 \\
\hline & 5194.0 \\
\hline $2^{\text {nd }}$ Fixed Grid Size $\left(\mathrm{N}_{\mathrm{CG}}\right)$ & 29460.5 \\
\hline $2^{\text {nd }}$ Total Grid Size $\left(\mathrm{N}_{\mathrm{FG}}\right)$ & \\
\hline & 29460.5 \\
\hline $3^{\text {rd }}$ Fixed Grid Size $\left(\mathrm{N}_{\mathrm{CG}}\right)$ & 100000 \\
\hline $3^{\text {rd }}$ Total Grid Size $\left(\mathrm{N}_{\mathrm{FG}}\right)$ & \\
\hline
\end{tabular}


Table XI shows how much dimensionless time was required to solve each of the grids and the total dimensionless time required.

Table XI. Dimensionless Time Required to Solve Nested-Grid Method

\begin{tabular}{|c|c|c|c|c|}
\hline $\begin{array}{c}\text { Course-Grid } \\
\text { Calculation }\end{array}$ & \multicolumn{3}{|c|}{ Nested-Grid Calculation } & \multirow{2}{*}{ Total Time } \\
\hline Coarse Grid & $1^{\text {st }}$ Nested Grid & $2^{\text {nd }}$ Nested Grid & $3^{\text {rd }}$ Nested Grid & \multirow{3}{*}{3129665.621} \\
\cline { 1 - 4 } Time & Time & Time & Time & \\
\cline { 1 - 4 } 59212.2201 & 228044.0282 & 737000.0365 & 2105409.336 & \\
\hline
\end{tabular}

Similar studies were conducted for grid sizes of $10^{3}$ and $10^{6}$. The results of all these studies are summarized in Figure 28 where the total dimensionless time required to obtain the finely-gridded pressure solution for a grid sizes of $10^{3}, 10^{5}$, and $10^{6}$ is plotted verses the number of nested grids used. It is evident from the plot that multiple nested grids have potential to reduce computational time even further.

Comparing the ratio of improvement in performance from a single nested grid, what was previously done in the study, to what was achieved when multiple nested grids were used is as follows. For the grid sizes of $10^{3}, 10^{5}$, and $10^{6}$ a factor of improvement of 2.1, 2.9, and 3.4 were noted respectively.

The ratio of improvement in dimensionless time for a single-grid solution to the largest number of nested-grids for the grid sizes of $10^{3}, 10^{5}$, and $10^{6}$ was a factor of 34.2 , 173.8, and 392.2. For all of the grid sizes, after the use of maximum number of nestedgrid systems shown in Figure 28, the total dimensionless time could not be determined using the Solver in Excel. It is clear from Figure 28 that as the number of nested grids increased, for all grid sizes considered, the overall dimensionless time reduction decreased and became insignificant. Consequently, one could achieve close the same 
dimensionless time reduction using two or three nested-grid systems as the maximum number of nested grids shown in the figure for all three grid sizes considered.

Multiple Nested-Grid Analysis

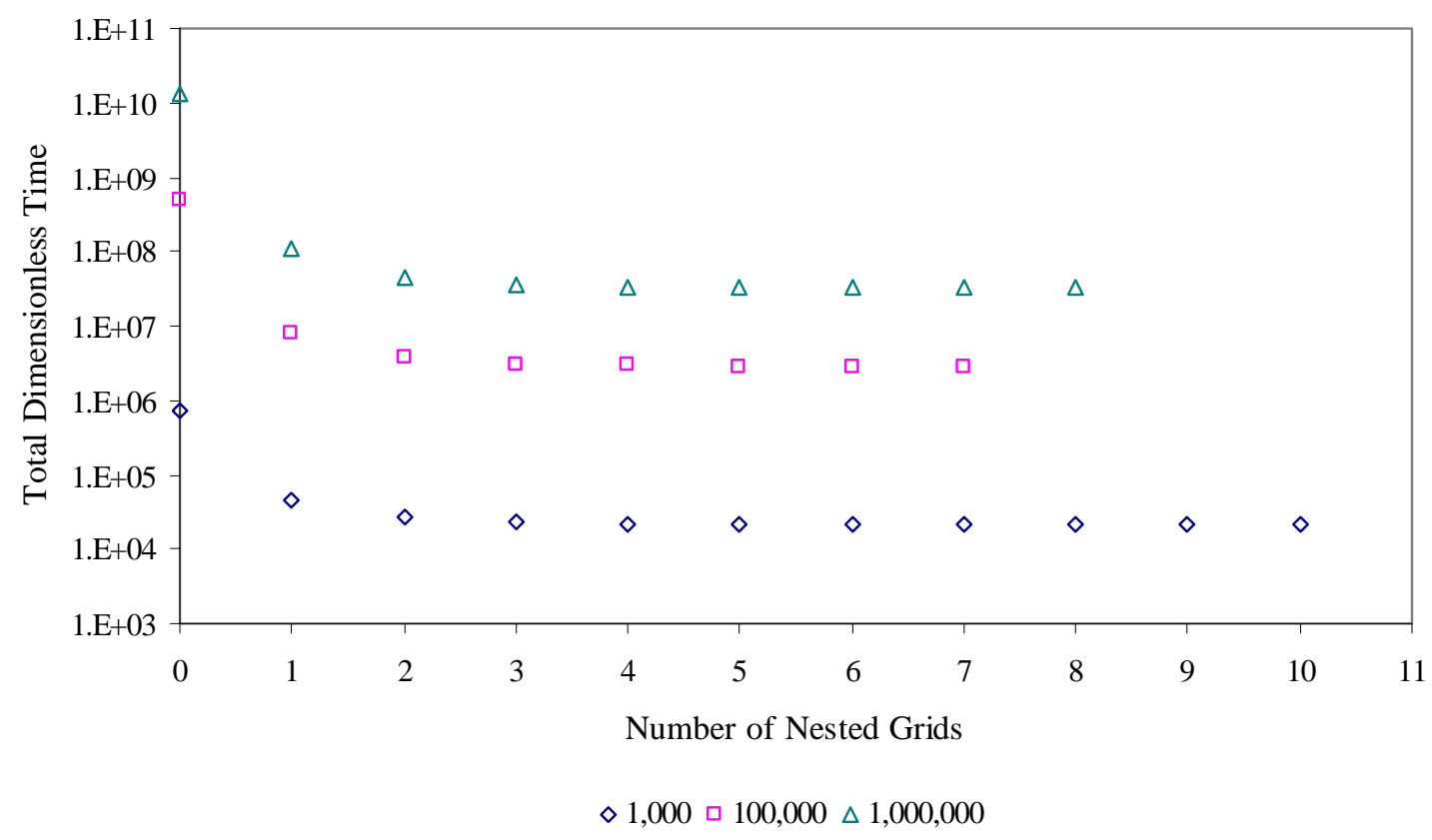

Figure 28. Multiple Nested-Grid Analysis: $10^{3}, 10^{5}$, and $10^{6}$ Grid Sizes

\subsection{Practical Multiple Nested-Grid Study}

The multiple nested-grid study to this point was essentially theoretical as the nested-grid systems could be realistically difficult to represent in a simulation while maintaining the integrity of the physical dimensions being studied. Due to this concern, a study was done where the nested-grid system was scaled using practical grid sizes. Practical nested-grid systems are systems where both coarse and fine girds have an integer number of grid points and the coarse grid points can be directly nested into the finer grid without compromising the physical reality of the reservoir being simulated. The total grid size calculated in the practical nested-grid study was a $1215 \times 1215 \times 2430$ grid or 
$3,587,226,750$ grid points. The original coarse grid calculated was a $5 \times 5 \times 10$ grid or 250 grid points. This grid was then nested into the successively larger grid of $15 \times 15 \times 30$, which was then nested in a 45x45x90 grid and so on until the final 1215x1215x2430 grid size was achieved. The dimensional time analysis results are shown in Figure 29. Each line represents the cumulative dimensionless time required to solve a given fine-grid. The top line, which is the dimensionless time without nested grids, indicates how long it takes to calculate each of the grid sizes using Weber SOR with two wells. The line underneath, which is the dimensionless time with practical nested grids, indicates how long it takes to calculate the same grid size using a practical setup of multiple nested-grids.

\section{Cumulative Dimensionless Time: Multiple Nested-Grids}

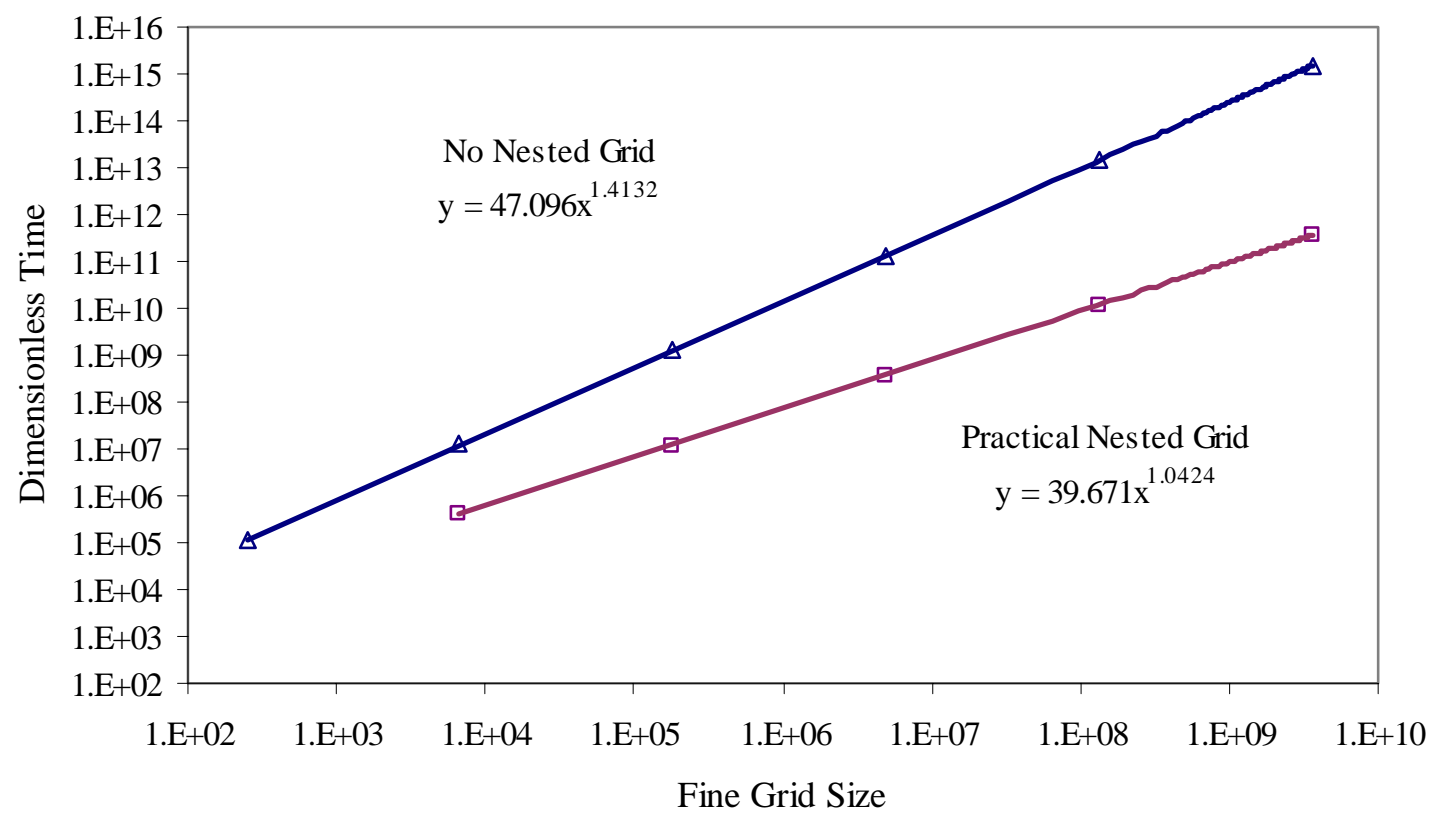

$\Delta$ No Nested Grid $\square$ Practical Nested Grid

Figure 29. Practical Nested-Grid Configuration 
This practical study shows a similar trend in the decrease of dimensionless time required to obtain the final finely-gridded pressure solution when optimized multiple nested-grids are used. It was clear that the use of multiple nested-grid systems was advantageous even when the system was not optimized.

A final plot compares the optimized, multiple nested-grid solution with the practical nested-grid solution and no nested-grid solution. The optimized grid configurations have the optimal theoretical number of nested-grid points.

Cumulative Dimensionless Time: Multiple Nested-Grids

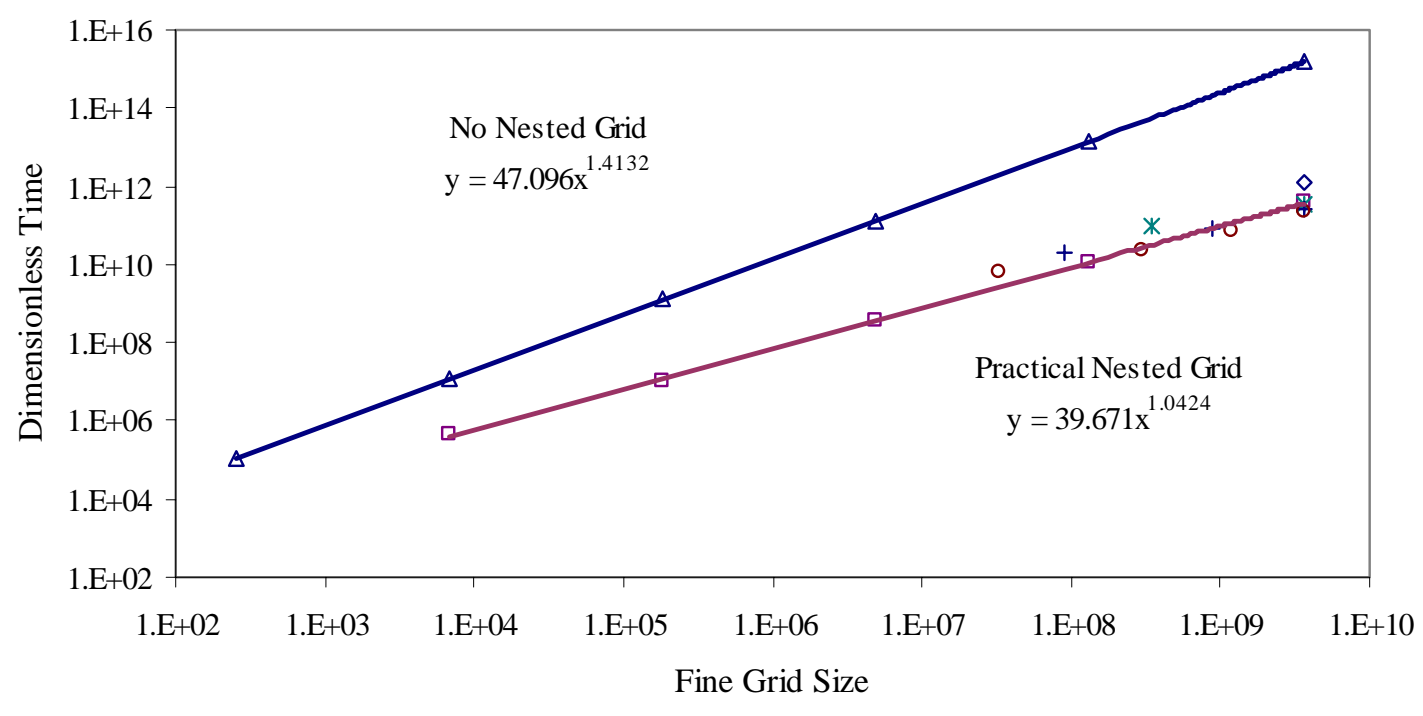

$\Delta$ No Nested Grid $\quad \square$ Practical Nested Grid

$\diamond$ One Optimized Nested-Grid System $\quad *$ Two Optimized Nested-Grid Systems

+ Three Optimized Nested-Grid Systems ○ Four Optimized Nested-Grid Systems

Figure 30. A Look at Optimal, Practical and No Nested Grid

The plot indicates that when the system of four multiple nested-grids was optimized the overall dimensionless time to calculate the finely-gridded pressure solution was reduced further than in the practical implementation of multiple nested-grids by 1.7 times. 
However, the practical implementation of the multiple nested-grid method would be the easiest to implement for a given reservoir.

\subsection{Summary}

The benefit of using multiple nested-grid systems to achieve the finely-gridded pressure solution was investigated. A theoretical study indicated improvements from a single-nested grid system ranging from a factor of about 2.1 on a grid size of $10^{3}$ to a factor of 3.4 on a grid size of $10^{6}$. In all the cases studied, using more than three nestedgrid systems was not useful in decreasing the dimensionless time further. The use of practical multiple nested-grids configurations was seen to perform essentially as well as the optimized nested-grid configurations. 


\section{CHAPTER 14}

\section{CONCLUSION AND FUTURE WORK}

\subsection{Conclusions}

Better technology is needed to produce oil and gas reserves in a more effective manner. Many developing technologies that improve oil and gas production would be greatly enhanced by faster and more accurate reservoir simulators. In approaching reservoir simulation, the multiscale method is an area of industry research which has been shown by various researchers to reduce the computational time required to complete reservoir simulations. Calculation of reservoir pressures is the most time consuming step in any reservoir simulator; this thesis proposes a new linear algebraic method that incorporates the multiscale approach to calculate reservoir simulation pressures in an accurate and fast manner.

Weber et. $\mathrm{al}^{27}$ developed finite-difference equations that incorporate physics of flow and allow for pressure solutions, four-orders-of-magnitude more accurate, to be generated on coarse grids. The proposed new calculation method developed in this thesis takes advantage of Weber's accurate coarse-grid solutions in a nested-grid calculation method to determine finely-gridded reservoir simulation pressures. Although this method incorporates some of the basic ideas of multigrid methods, namely coarse-grid relaxation and nested iteration, it is not a multigrid method. The coarse-grid pressure 
solutions are nested into a finer grid and fixed throughout the relaxation process on the desired fine grid. The reservoir being simulated is of an idealized nature. The simplify assumptions include: (1) homogeneous permeability, (2) neglected gravity effects, (3) incompressibility, (4) steady-state, and (5) dimensions of reservoir and placement of wells generate a symmetric pressure solution.

A standard desktop computer was used for the study of the performance of various solvers as a function of gird size. Direct solution methods considered were Gauss Elimination $^{29}$, the Doolittle LU factorization method $^{29}$, and a band solver, DGBSV, from the LAPACK library. ${ }^{30}$ Stationary iterative methods considered for the study were Jacobi, Gauss-Seidel, and Successive-Over-Relaxation (SOR). Five nonstationary iterative methods from MATLAB 7.0: Biconjugate Gradient (BICG), Biconjugate Gradient stabilized (BICGSTAB), LSQR implementation of Conjugate Gradients on the Normal Equations (LSQR), Generalized Minimum Residual (GMRES), and Quasiminimal Residual (QMR) were considered. A hybrid of direct and iterative methods, Line Successive-Over-Relaxation (LSOR), was also considered. SOR and LSOR required the determination of an optimal over-relaxation factor.

Of the direct methods tested, the band solver, DGBSV, from the LAPACK library showed best performance with respect to both cpu time and memory requirements. LSOR showed the best cpu time performance of the stationary and nonstationary iterative methods, with SOR following closely behind; SOR and Gauss Seidel required the least amount of run-time memory of all iterative methods.

SOR was determined to be the best solver for the next phase of the study. It had similar convergence time to LSOR and its memory requirements were much lower. SOR 
had similar performance to the best direct method on the smallest grid size considered and outperformed any direct method on all larger grids. Even though their overall performance parameters were not as good as SOR, GMRES and LSOR were also considered for implementation in the nested-grid method.

The nested-grid method was implemented using various nested-grid configurations. LaPlace SOR and GMRES were used to calculate the finely-gridded pressures on the nested-grid configurations and performance data was recorded (See Tables A.XIII and A.XIV). Significant improvements in the time required for convergence were noted for both LaPlace SOR and GMRES, as nested-pressures were fixed in the fine-grid. These improvements gave a strong indication that Weber's accurate pressure-solutions could be implemented in a nested-grid method and reduce computation time required to achieve finely-gridded reservoir simulation pressures.

In an effort to speed up the solution method further, a three-dimensional linear interpolation of the nested-grid configuration was computed. Interpolation was not found to reduce the cpu time required to obtain the finely-gridded pressures in a significant manner. The lack of significant improvement using interpolation emphasizes the impact that the optimal over-relaxation factor has on the solution of a system of equations. It was thought that the interpolation program would possibly improve the solution method, yet for interpolation in three dimensions it became clear that the time required to simply interpolate values for every grid point added enough time to the overall solution method to make it an undesirable algorithm step. For this reason, interpolation was not recommended for further use in the study. 
The math of Weber et al. ${ }^{27}$ is summarized in Chapter 8 . The end result is six lambda (Weber) coefficients that can be easily incorporated into traditional finitedifference formulations to calculate accurate pressure solutions. Weber's coefficient need only be calculated for the interior cell faces of a block of cells around the well. For the coarsest grid considered, a block of twenty-seven cells was used which resulted in the calculation of eighteen coefficients in each direction. Finer grids required the calculation of more coefficients, as the same pattern was applied over a larger number of grid cells.

The nested-grid study, applied with Weber's coefficients, was conducted in a very similar manner to that discussed in Chapter 6. Fine-grid sizes that were odd multiples of the original coarse-grid size allowed for the accurate coarse-grid pressure solutions of Weber to be nested into desired fine grids. Various nested-grid configurations were considered and the resulting performance data was recorded (See Table A.XVII).

The performance data from LaPlace SOR, Weber SOR, and GMRES were regressed to generate mathematical correlations that could be used to predict the number of iterations required for convergence as well as the optimal over-relaxation factor for any nested-grid configuration. The correlations predicted the actual values of number of iterations required for convergence and the over-relaxation factor very well. These correlations were used in the dimensionless time analysis of Chapter 11.

For LaPlace SOR, GMRES, and Weber SOR the time/iteration, determined from the performance data, was observed to vary more or less directly with total grid size. This allowed for a dimensionless cpu time expression to be developed which incorporated the mathematical correlations described in Chapter 10 and represented the actual cpu time required for convergence of a particular nested-grid configuration. The dimensionless 
time expression was minimized, by optimizing the number of coarse-grid points in any given nested-grid setup. The results showed significant reductions in the amount of computational time required to obtain finely-gridded pressures solutions. The ratio of dimensionless time improvement for Weber SOR ranged from 7 on the coarsest nestedgrid setup to 813 on the finest nested-grid. This verified that the solution method developed in this thesis generates fast, accurate pressure solutions on fine grids.

Two measurements, absolute difference and absolute percentage difference from a one-step solution, were made to determine the amount of error inherent in the nested-grid method applied with Weber's equations. The absolute difference values showed that for the grid sizes considered, the maximum absolute difference never exceeds 0.1902 and the average absolute difference never exceeds 0.0168. These errors are of the same magnitude found by Weber (0.0757 maximum and 0.0182 average) and demonstrate that the nested-grid solution method does not substantially decrease the extraordinary accuracy of his solution. For the coarsest grid used, the maximum percent difference recorded was under 5\%. This may be disconcerting, but potential remedies for this would be using a finer coarse grid, using more realistic well pressures that do not generate a symmetric solution or using a more rigorous convergence criterion.

The benefit of using multiple nested-grid systems to achieve the finely-gridded pressure solution was investigated. A theoretical study indicated improvements from a single-nested grid system ranging from a factor of about 2.1 on a grid size of $10^{3}$ to a factor of 3.4 on a grid size of $10^{6}$. In all cases studied, using more than three nested-grid systems was not useful in decreasing the dimensionless time further. The use of practical 
multiple nested-grids configurations was seen to perform essentially as well as the optimized nested-grid configurations.

The nested-grid method dramatically improves the speed at which a solution of finely-gridded pressures can be generated. Keys to the success of this new method were the new finite-difference equations that incorporated the physics of the flow, the placement of an optimal number of nested-grid points into the fine grid, and the use of an optimal number of nested-grid systems. The end result was an accurate and fast solution method.

\subsection{Future Work}

Recommended future work involves investigating the effect of permeability variations on the solution method, as the cases studied were of an idealized system. This would involve the investigation of heterogeneous reservoirs and might be approached through a series of larger and smaller nested grids similar to multigrid methods, or by judicious choice of scaled-up coefficients. In addition, the work that was initiated in this thesis and described in Appendix D, which initiates the development of an algorithm that can determine the optimal over-relaxation factor, used in Successive-Over-Relaxation, for any grid, should be continued. Weber's work is valid only for wells that can be approximated by points or straight lines. The wells must be centered in the grid cells. This work should be extended to include wells of arbitrary shape and location. These new technologies should then be used to create a computer program that can be used to solve the pressure equation in reservoir simulators throughout the petroleum industry. 
APPENDIX 


\section{APPENDIX A}

\section{TABLES REFERRED TO IN BODY OF THESIS}

Table A.XII. Nested-Grid Configurations

\begin{tabular}{|c|c|c|c|c|}
\hline $\begin{array}{c}\text { Fine-Grid } \\
\text { Dimensions }\end{array}$ & $\begin{array}{c}\text { Fine- } \\
\text { Grid } \\
\text { Size }\end{array}$ & $\begin{array}{c}\text { Coarse- } \\
\text { Grid Size }\end{array}$ & $\begin{array}{c}\text { Number of } \\
\text { Fine-Grid } \\
\text { Points } \\
\text { Between } \\
\text { Coarse-Grid } \\
\text { Points }\end{array}$ & $\begin{array}{c}\text { Percent of Fixed } \\
\text { Points }\end{array}$ \\
\hline 5X5X10 & 250 & 18 & 1 & 6.4000 \\
\hline 9X9X18 & 1458 & 18 & 3 & 1.0974 \\
\hline 17X17X34 & 9826 & 18 & 7 & 0.1628 \\
\hline 33X33X66 & 71874 & 18 & 15 & 0.0223 \\
\hline 65X65X130 & 549250 & 18 & 31 & 0.0029 \\
\hline 9X9X18 & 1458 & 130 & 1 & 8.7791 \\
\hline 17X17X34 & 9826 & 130 & 3 & 1.3027 \\
\hline 33X33X66 & 71874 & 130 & 7 & 0.1781 \\
\hline 65X65X130 & 549250 & 130 & 15 & 0.0233 \\
\hline 17X17X34 & 9826 & 1026 & 1 & 10.4213 \\
\hline 33X33X66 & 71874 & 1026 & 3 & 1.4247 \\
\hline $65 X 65 X 130$ & 549250 & 1026 & 7 & 0.1868 \\
\hline 33X33X66 & 71874 & 8194 & 1 & 11.3977 \\
\hline 65X65X130 & 549250 & 8194 & 3 & 1.4915 \\
\hline 65X65X130 & 549250 & 65538 & 1 & 11.9319 \\
\hline
\end{tabular}

Table A.XIII. LaPlace SOR: Fine and Nested-Grid Results

\begin{tabular}{|c|c|c|c|c|c|}
\hline $\mathrm{N}_{\mathrm{FG}}$ & $\mathrm{N}_{\mathrm{CG}}$ & $\omega_{\text {opt }}$ & $\mathrm{N}_{\text {ITER }}$ & Time(s) & Time/Iter \\
\hline 250 & 2 & 1.800 & 82 & 0.007 & $8.61 \mathrm{E}-05$ \\
\hline 1458 & 2 & 1.910 & 255 & 0.086 & $3.36 \mathrm{E}-04$ \\
\hline 9826 & 2 & 1.968 & 546 & 1.179 & $2.16 \mathrm{E}-03$ \\
\hline 71874 & 2 & 1.988 & 1104 & 33.562 & $3.04 \mathrm{E}-02$ \\
\hline
\end{tabular}


Table A.XIII. continued

\begin{tabular}{|c|c|c|c|c|c|}
\hline 549250 & 2 & 1.996 & 3356 & 881.925 & $2.63 \mathrm{E}-01$ \\
\hline 250 & 18 & 1.490 & 32 & 0.003 & $1.41 \mathrm{E}-04$ \\
\hline 1458 & 18 & 1.780 & 58 & 0.019 & $3.24 \mathrm{E}-04$ \\
\hline 9826 & 18 & 1.910 & 182 & 0.410 & $2.19 \mathrm{E}-03$ \\
\hline 71874 & 18 & 1.969 & 483 & 15.328 & $3.26 \mathrm{E}-02$ \\
\hline 549250 & 18 & 1.989 & 902 & 242.160 & $2.68 \mathrm{E}-01$ \\
\hline 1458 & 130 & 1.460 & 37 & 0.019 & $9.54 \mathrm{E}-05$ \\
\hline 9826 & 130 & 1.751 & 79 & 0.191 & $2.42 \mathrm{E}-03$ \\
\hline 71874 & 130 & 1.912 & 212 & 7.081 & $3.40 \mathrm{E}-02$ \\
\hline 549250 & 130 & 1.971 & 533 & 145.910 & $2.74 \mathrm{E}-01$ \\
\hline 9826 & 1026 & 1.410 & 35 & 0.082 & $2.87 \mathrm{E}-03$ \\
\hline 71874 & 1026 & 1.770 & 85 & 2.941 & $3.45 \mathrm{E}-02$ \\
\hline 549250 & 1026 & 1.920 & 257 & 73.582 & $2.92 \mathrm{E}-01$ \\
\hline 71874 & 8194 & 1.430 & 35 & 1.223 & $3.46 \mathrm{E}-02$ \\
\hline 549250 & 8194 & 1.760 & 94 & 28.425 & $2.99 \mathrm{E}-01$ \\
\hline 549250 & 65538 & 1.430 & 37 & 12.160 & $3.28 \mathrm{E}-01$ \\
\hline
\end{tabular}

Table A.XIV. GMRES: Fine and Nested-Grid Results

\begin{tabular}{|c|c|c|c|c|}
\hline $\mathrm{N}_{\mathrm{FG}}$ & $\mathrm{N}_{\mathrm{CG}}$ & $\mathrm{N}_{\text {ITER }}$ & Time(s) & Time/Iter \\
\hline 250 & 2 & 22 & 0.063 & $2.84 \mathrm{E}-03$ \\
\hline 1458 & 2 & 42 & 0.322 & $7.66 \mathrm{E}-03$ \\
\hline 9826 & 2 & 80 & 3.153 & $3.94 \mathrm{E}-02$ \\
\hline 71874 & 2 & - & - & - \\
\hline 549250 & 2 & - & - & - \\
\hline 250 & 18 & 20 & 0.063 & $3.13 \mathrm{E}-03$ \\
\hline 1458 & 18 & 40 & 0.294 & $7.34 \mathrm{E}-03$ \\
\hline 9826 & 18 & 80 & 4.966 & $6.21 \mathrm{E}-02$ \\
\hline 71874 & 18 & 155 & 355.688 & $2.29 \mathrm{E}+00$ \\
\hline 549250 & 18 & - & - & - \\
\hline 1458 & 130 & 30 & 0.219 & $7.29 \mathrm{E}-03$ \\
\hline 9826 & 130 & 67 & 3.225 & $4.81 \mathrm{E}-02$ \\
\hline 71874 & 130 & 137 & 289.081 & $2.11 \mathrm{E}+00$ \\
\hline 549250 & 130 & - & - & - \\
\hline 9826 & 1026 & 36 & 1.494 & $4.15 \mathrm{E}-02$ \\
\hline 71874 & 1026 & 89 & 123.544 & $1.39 \mathrm{E}+00$ \\
\hline 549250 & 1026 & - & - & - \\
\hline 71874 & 8194 & 36 & 25.534 & $7.09 \mathrm{E}-01$ \\
\hline 549250 & 8194 & - & - & - \\
\hline 549250 & 65538 & - & - & - \\
\hline \multicolumn{5}{|r}{} \\
\hline
\end{tabular}


Table A.XV. Interpolation Study

\begin{tabular}{|c|c|c|c|c|}
\hline $\mathrm{N}_{\mathrm{FG}}$ & $\mathrm{N}_{\mathrm{CG}}$ & $\begin{array}{c}\text { Time to } \\
\text { Interpolate } \\
\text { Nested-Grid (s) }\end{array}$ & $\begin{array}{c}\text { Time to Iterate to Convergence } \\
\text { from Interpolated Starting } \\
\text { Values (s) }\end{array}$ & Total Time (s) \\
\hline 250 & 18 & 0.010 & 0.003 & 0.013 \\
\hline 1458 & 18 & 0.019 & 0.029 & 0.048 \\
\hline 9826 & 18 & 0.140 & 0.430 & 0.570 \\
\hline 71874 & 18 & 1.016 & 14.288 & 15.304 \\
\hline 549250 & 18 & 7.424 & 364.785 & 372.209 \\
\hline 1458 & 130 & 0.021 & 0.023 & 0.044 \\
\hline 9826 & 130 & 0.144 & 0.187 & 0.331 \\
\hline 71874 & 130 & 1.019 & 6.351 & 7.370 \\
\hline 549250 & 130 & 7.380 & 123.351 & 130.732 \\
\hline 9826 & 1026 & 0.131 & 0.085 & 0.216 \\
\hline 71874 & 1026 & 1.016 & 2.507 & 3.523 \\
\hline 549250 & 1026 & 7.355 & 62.531 & 69.886 \\
\hline 71874 & 8194 & 0.962 & 0.805 & 1.767 \\
\hline 549250 & 8194 & 7.324 & 13.539 & 20.863 \\
\hline 549250 & 65538 & 6.861 & 4.937 & 11.798 \\
\hline
\end{tabular}

Table A.XVI. Interpolation Study: Comparison with No Interpolation

\begin{tabular}{|c|c|c|c|c|}
\hline $\mathrm{N}_{\mathrm{FG}}$ & $\mathrm{N}_{\mathrm{CG}}$ & $\begin{array}{c}\text { Time Required to } \\
\text { Compute Pressure } \\
\text { Solution with } \\
\text { Interpolation (s) }\end{array}$ & $\begin{array}{c}\text { Time Required to } \\
\text { Compute Pressure } \\
\text { Solution Without } \\
\text { Interpolation (s) }\end{array}$ & $\begin{array}{c}\text { Ratio of } \\
\text { Interpolation to No } \\
\text { Interpolation }\end{array}$ \\
\hline 250 & 18 & $1.32 \mathrm{E}-02$ & $3.16 \mathrm{E}-03$ & 4.20 \\
\hline 1458 & 18 & $4.82 \mathrm{E}-02$ & $1.90 \mathrm{E}-02$ & 2.53 \\
\hline 9826 & 18 & $5.70 \mathrm{E}-01$ & $4.10 \mathrm{E}-01$ & 1.39 \\
\hline 71874 & 18 & $1.53 \mathrm{E}+01$ & $1.53 \mathrm{E}+01$ & 1.00 \\
\hline 549250 & 18 & $3.72 \mathrm{E}+02$ & $2.42 \mathrm{E}+02$ & 1.54 \\
\hline 1458 & 130 & $4.42 \mathrm{E}-02$ & $1.93 \mathrm{E}-02$ & 2.29 \\
\hline 9826 & 130 & $3.31 \mathrm{E}-01$ & $1.91 \mathrm{E}-01$ & 1.73 \\
\hline 71874 & 130 & $7.37 \mathrm{E}+00$ & $7.08 \mathrm{E}+00$ & 1.04 \\
\hline 549250 & 130 & $1.31 \mathrm{E}+02$ & $1.46 \mathrm{E}+02$ & 0.90 \\
\hline 9826 & 1026 & $2.16 \mathrm{E}-01$ & $8.18 \mathrm{E}-02$ & 2.65 \\
\hline 71874 & 1026 & $3.52 \mathrm{E}+00$ & $2.94 \mathrm{E}+00$ & 1.20 \\
\hline 549250 & 1026 & $6.99 \mathrm{E}+01$ & $7.36 \mathrm{E}+01$ & 0.95 \\
\hline 71874 & 8194 & $1.77 \mathrm{E}+00$ & $1.22 \mathrm{E}+00$ & 1.44 \\
\hline 549250 & 8194 & $2.09 \mathrm{E}+01$ & $2.84 \mathrm{E}+01$ & 0.73 \\
\hline 549250 & 65538 & $1.18 \mathrm{E}+01$ & $1.22 \mathrm{E}+01$ & 0.97 \\
\hline
\end{tabular}


Table A.XVII. Weber SOR: Fine and Nested-Grid Results with Weber's Coefficients

\begin{tabular}{|c|c|c|c|c|c|c|}
\hline Dimensions & $\mathrm{N}_{\mathrm{FG}}$ & $\mathrm{N}_{\mathrm{CG}}$ & $\omega_{\text {opt }}$ & $\mathrm{N}_{\text {ITER }}$ & Time(s) & Time/Iter \\
\hline $5 \times 5 \times 10$ & 250 & 2 & 1.9831 & 634 & 4.947E-02 & 7.803E-05 \\
\hline $15 \times 15 \times 30$ & 6750 & 2 & 1.9947 & 1875 & $4.489 \mathrm{E}+00$ & $2.394 \mathrm{E}-03$ \\
\hline $25 \times 25 \times 50$ & 31250 & 2 & 1.9975 & 3410 & $1.249 \mathrm{E}+02$ & $3.661 \mathrm{E}-02$ \\
\hline $35 \times 35 \times 70$ & 85750 & 2 & 1.9977 & 4762 & $5.457 \mathrm{E}+02$ & 1.146E-01 \\
\hline $45 \times 45 \times 90$ & 182250 & 2 & 1.9982 & 6370 & $1.640 \mathrm{E}+03$ & 2.575E-01 \\
\hline $55 \times 55 \times 110$ & 332750 & 2 & 1.9985 & 8284 & $3.785 E+03$ & 4.569E-01 \\
\hline $65 \times 65 \times 130$ & 549250 & 2 & 1.9989 & 10371 & $8.036 \mathrm{E}+03$ & 7.749E-01 \\
\hline $75 \times 75 \times 150$ & 843750 & 2 & 1.999 & 13057 & $1.570 \mathrm{E}+04$ & $1.203 \mathrm{E}+00$ \\
\hline $5 \times 5 \times 10$ & 250 & 18 & 1.47 & 25 & $1.875 \mathrm{E}-03$ & $7.500 \mathrm{E}-05$ \\
\hline $15 \times 15 \times 30$ & 6750 & 18 & 1.89 & 115 & 3.042E-01 & $2.645 \mathrm{E}-03$ \\
\hline $25 \times 25 \times 50$ & 31250 & 18 & 1.96 & 250 & $1.576 \mathrm{E}+01$ & 6.305E-02 \\
\hline $35 \times 35 \times 70$ & 85750 & 18 & 1.974 & 361 & $6.591 \mathrm{E}+01$ & 1.826E-01 \\
\hline $45 \times 45 \times 90$ & 182250 & 18 & 1.9795 & 470 & $1.163 \mathrm{E}+02$ & 2.475E-01 \\
\hline $55 \times 55 \times 110$ & 332750 & 18 & 1.9851 & 584 & $2.668 \mathrm{E}+02$ & 4.568E-01 \\
\hline $65 \times 65 \times 130$ & 549250 & 18 & 1.9888 & 536 & $4.328 \mathrm{E}+02$ & $8.075 \mathrm{E}-01$ \\
\hline $75 \times 75 \times 150$ & 843750 & 18 & 1.9912 & 1209 & $1.535 \mathrm{E}+03$ & $1.270 \mathrm{E}+00$ \\
\hline $15 \times 15 \times 30$ & 6750 & 54 & 1.81 & 75 & 2.156E-01 & 2.874E-03 \\
\hline $25 \times 25 \times 50$ & 31250 & 54 & 1.911 & 142 & $9.063 \mathrm{E}+00$ & $6.382 \mathrm{E}-02$ \\
\hline $35 \times 35 \times 70$ & 85750 & 54 & 1.951 & 214 & $4.000 \mathrm{E}+01$ & 1.869E-01 \\
\hline $45 \times 45 \times 90$ & 182250 & 54 & 1.963 & 315 & $7.966 \mathrm{E}+01$ & 2.529E-01 \\
\hline $55 \times 55 \times 110$ & 332750 & 54 & 1.974 & 340 & $1.586 \mathrm{E}+02$ & 4.664E-01 \\
\hline $65 \times 65 \times 130$ & 549250 & 54 & 1.981 & 263 & $2.113 \mathrm{E}+02$ & 8.034E-01 \\
\hline $75 \times 75 \times 150$ & 843750 & 54 & & & & \\
\hline $15 \times 15 \times 30$ & 6750 & 130 & 1.75 & 55 & $1.262 \mathrm{E}-01$ & 2.294E-03 \\
\hline $45 \times 45 \times 90$ & 182250 & 130 & 1.94 & 254 & $6.455 \mathrm{E}+01$ & $2.541 \mathrm{E}-01$ \\
\hline $75 \times 75 \times 150$ & 843750 & 130 & & & & \\
\hline 15x15x30 & 6750 & 250 & 1.678 & 41 & $1.045 \mathrm{E}-01$ & $2.549 \mathrm{E}-03$ \\
\hline $25 \times 25 \times 50$ & 31250 & 250 & 1.829 & 94 & $6.262 \mathrm{E}+00$ & 6.662E-02 \\
\hline $35 \times 35 \times 70$ & 85750 & 250 & 1.883 & 128 & $2.411 \mathrm{E}+01$ & 1.884E-01 \\
\hline $45 \times 45 \times 90$ & 182250 & 250 & 1.9159 & 183 & $4.760 \mathrm{E}+01$ & 2.601E-01 \\
\hline $55 \times 55 \times 110$ & 332750 & 250 & 1.9513 & 277 & $1.324 \mathrm{E}+02$ & $4.780 \mathrm{E}-01$ \\
\hline $65 \times 65 \times 130$ & 549250 & 250 & 1.9529 & 289 & $2.372 \mathrm{E}+02$ & 8.207E-01 \\
\hline $75 \times 75 \times 150$ & 843750 & 250 & 1.9617 & 347 & $4.369 \mathrm{E}+02$ & $1.259 \mathrm{E}+00$ \\
\hline $45 \times 45 \times 90$ & 182250 & 1026 & 1.817 & 123 & $3.141 \mathrm{E}+01$ & $2.554 \mathrm{E}-01$ \\
\hline $75 \times 75 \times 150$ & 843750 & 1026 & & & & \\
\hline $45 \times 45 \times 90$ & 182250 & 6750 & 1.63 & 48 & $1.224 \mathrm{E}+01$ & 2.550E-01 \\
\hline $75 \times 75 \times 150$ & 843750 & 6750 & & & & \\
\hline $75 \times 75 \times 150$ & 843750 & 31250 & 1.631 & 48 & $6.062 \mathrm{E}+01$ & $1.263 \mathrm{E}+00$ \\
\hline
\end{tabular}


Table A.XVIII. LaPlace SOR: Dimensionless Time Results of Nested-Grid Method

\begin{tabular}{|c|c|c|c|c|}
\hline $\begin{array}{c}\text { Optimal } \\
\mathrm{N}_{\mathrm{CG}}\end{array}$ & $\mathrm{N}_{\mathrm{FG}}$ & $\begin{array}{c}\text { Dimensionless } \\
\text { Time: Two } \\
\text { Fixed Points }\end{array}$ & $\begin{array}{c}\text { Dimensionless } \\
\text { Time: Optimal } \\
\text { Fixed Points }\end{array}$ & $\begin{array}{c}\text { Ratio of } \\
\text { Improvement }\end{array}$ \\
\hline 11 & $5.00 \mathrm{E}+01$ & $2.78 \mathrm{E}+03$ & $1.15 \mathrm{E}+03$ & 2 \\
\hline 18 & $1.00 \mathrm{E}+02$ & $7.57 \mathrm{E}+03$ & $2.59 \mathrm{E}+03$ & 3 \\
\hline 36 & $2.50 \mathrm{E}+02$ & $2.80 \mathrm{E}+04$ & $7.47 \mathrm{E}+03$ & 4 \\
\hline 61 & $5.00 \mathrm{E}+02$ & $7.50 \mathrm{E}+04$ & $1.65 \mathrm{E}+04$ & 5 \\
\hline 104 & $1.00 \mathrm{E}+03$ & $2.00 \mathrm{E}+05$ & $3.65 \mathrm{E}+04$ & 5 \\
\hline 140 & $1.46 \mathrm{E}+03$ & $3.41 \mathrm{E}+05$ & $5.60 \mathrm{E}+04$ & 6 \\
\hline 369 & $5.00 \mathrm{E}+03$ & $1.95 \mathrm{E}+06$ & $2.27 \mathrm{E}+05$ & 9 \\
\hline 630 & $9.83 \mathrm{E}+03$ & $5.06 \mathrm{E}+06$ & $4.90 \mathrm{E}+05$ & 10 \\
\hline 1322 & $2.50 \mathrm{E}+04$ & $1.89 \mathrm{E}+07$ & $1.41 \mathrm{E}+06$ & 13 \\
\hline 2293 & $5.00 \mathrm{E}+04$ & $5.04 \mathrm{E}+07$ & $3.10 \mathrm{E}+06$ & 16 \\
\hline 3060 & $7.19 \mathrm{E}+04$ & $8.42 \mathrm{E}+07$ & $4.68 \mathrm{E}+06$ & 18 \\
\hline 15469 & $5.49 \mathrm{E}+05$ & $1.49 \mathrm{E}+09$ & $4.68 \mathrm{E}+07$ & 32 \\
\hline 24948 & $1.00 \mathrm{E}+06$ & $3.47 \mathrm{E}+09$ & $9.22 \mathrm{E}+07$ & 38 \\
\hline 90139 & $5.00 \mathrm{E}+06$ & $3.37 \mathrm{E}+10$ & $5.70 \mathrm{E}+08$ & 59 \\
\hline 156786 & $1.00 \mathrm{E}+07$ & $8.98 \mathrm{E}+10$ & $1.25 \mathrm{E}+09$ & 72 \\
\hline 986868 & $1.00 \mathrm{E}+08$ & $2.32 \mathrm{E}+12$ & $1.69 \mathrm{E}+10$ & 138 \\
\hline 6217592 & $1.00 \mathrm{E}+09$ & $6.01 \mathrm{E}+13$ & $2.28 \mathrm{E}+11$ & 263 \\
\hline
\end{tabular}

Table A.XIX. GMRES: Dimensionless Time Results of Nested-Grid Method

\begin{tabular}{|c|c|c|c|c|}
\hline $\begin{array}{c}\text { Optimal } \\
\mathrm{N}_{\mathrm{CG}}\end{array}$ & $\mathrm{N}_{\mathrm{FG}}$ & $\begin{array}{c}\text { Dimensionless } \\
\text { Time: Two } \\
\text { Fixed Points }\end{array}$ & $\begin{array}{c}\text { Dimensionless } \\
\text { Time: Optimal } \\
\text { Fixed Points }\end{array}$ & $\begin{array}{c}\text { Ratio of } \\
\text { Improvement }\end{array}$ \\
\hline 7 & $1.00 \mathrm{E}+02$ & $3.00 \mathrm{E}+04$ & $2.99 \mathrm{E}+04$ & 1 \\
\hline 36 & $2.50 \mathrm{E}+02$ & $1.05 \mathrm{E}+05$ & $1.02 \mathrm{E}+05$ & 1 \\
\hline 112 & $5.00 \mathrm{E}+02$ & $2.69 \mathrm{E}+05$ & $2.50 \mathrm{E}+05$ & 1 \\
\hline 274 & $1.00 \mathrm{E}+03$ & $6.90 \mathrm{E}+05$ & $5.84 \mathrm{E}+05$ & 1 \\
\hline 411 & $1.46 \mathrm{E}+03$ & $1.15 \mathrm{E}+06$ & $9.09 \mathrm{E}+05$ & 1 \\
\hline 1271 & $5.00 \mathrm{E}+03$ & $6.16 \mathrm{E}+06$ & $3.62 \mathrm{E}+06$ & 2 \\
\hline 2210 & $9.83 \mathrm{E}+03$ & $1.54 \mathrm{E}+07$ & $7.50 \mathrm{E}+06$ & 2 \\
\hline 4590 & $2.50 \mathrm{E}+04$ & $5.49 \mathrm{E}+07$ & $2.01 \mathrm{E}+07$ & 3 \\
\hline 7790 & $5.00 \mathrm{E}+04$ & $1.41 \mathrm{E}+08$ & $4.15 \mathrm{E}+07$ & 3 \\
\hline 10249 & $7.19 \mathrm{E}+04$ & $2.31 \mathrm{E}+08$ & $6.04 \mathrm{E}+07$ & 4 \\
\hline 47000 & $5.49 \mathrm{E}+05$ & $3.66 \mathrm{E}+09$ & $4.90 \mathrm{E}+08$ & 7 \\
\hline 73485 & $1.00 \mathrm{E}+06$ & $8.27 \mathrm{E}+09$ & $9.05 \mathrm{E}+08$ & 9 \\
\hline 243985 & $5.00 \mathrm{E}+06$ & $7.37 \mathrm{E}+10$ & $4.69 \mathrm{E}+09$ & 16 \\
\hline 409156 & $1.00 \mathrm{E}+07$ & $1.89 \mathrm{E}+11$ & $9.51 \mathrm{E}+09$ & 20 \\
\hline
\end{tabular}


Table A.XIX. continued

\begin{tabular}{|c|c|c|c|c|}
\hline 2281845 & $1.00 \mathrm{E}+08$ & $4.33 \mathrm{E}+12$ & $9.94 \mathrm{E}+10$ & 44 \\
\hline 12746216 & $1.00 \mathrm{E}+09$ & $9.89 \mathrm{E}+13$ & $1.04 \mathrm{E}+12$ & 95 \\
\hline
\end{tabular}

Table A.XX. Weber SOR: Dimensionless Time Results of Nested-Grid Method

\begin{tabular}{|c|c|c|c|c|}
\hline $\begin{array}{c}\text { Optimal } \\
\mathrm{N}_{\mathrm{CG}}\end{array}$ & $\mathrm{N}_{\mathrm{FG}}$ & $\begin{array}{c}\text { Dimensionless } \\
\text { Time: Two } \\
\text { Fixed Points }\end{array}$ & $\begin{array}{c}\text { Dimensionless } \\
\text { Time: Optimal } \\
\text { Fixed Points }\end{array}$ & $\begin{array}{c}\text { Ratio of } \\
\text { Improvement }\end{array}$ \\
\hline 6 & $5.00 \mathrm{E}+01$ & $1.01 \mathrm{E}+04$ & $1.42 \mathrm{E}+03$ & 7 \\
\hline 9 & $1.00 \mathrm{E}+02$ & $2.78 \mathrm{E}+04$ & $3.19 \mathrm{E}+03$ & 9 \\
\hline 17 & $2.50 \mathrm{E}+02$ & $1.03 \mathrm{E}+05$ & $9.15 \mathrm{E}+03$ & 11 \\
\hline 28 & $5.00 \mathrm{E}+02$ & $2.76 \mathrm{E}+05$ & $2.02 \mathrm{E}+04$ & 14 \\
\hline 47 & $1.00 \mathrm{E}+03$ & $7.38 \mathrm{E}+05$ & $4.44 \mathrm{E}+04$ & 17 \\
\hline 63 & $1.46 \mathrm{E}+03$ & $1.26 \mathrm{E}+06$ & $6.80 \mathrm{E}+04$ & 18 \\
\hline 164 & $5.00 \mathrm{E}+03$ & $7.19 \mathrm{E}+06$ & $2.75 \mathrm{E}+05$ & 26 \\
\hline 280 & $9.83 \mathrm{E}+03$ & $1.87 \mathrm{E}+07$ & $5.91 \mathrm{E}+05$ & 32 \\
\hline 588 & $2.50 \mathrm{E}+04$ & $6.98 \mathrm{E}+07$ & $1.70 \mathrm{E}+06$ & 41 \\
\hline 1021 & $5.00 \mathrm{E}+04$ & $1.86 \mathrm{E}+08$ & $3.73 \mathrm{E}+06$ & 50 \\
\hline 1364 & $7.19 \mathrm{E}+04$ & $3.10 \mathrm{E}+08$ & $5.62 \mathrm{E}+06$ & 55 \\
\hline 6913 & $5.49 \mathrm{E}+05$ & $5.49 \mathrm{E}+09$ & $5.61 \mathrm{E}+07$ & 98 \\
\hline 11157 & $1.00 \mathrm{E}+06$ & $1.28 \mathrm{E}+10$ & $1.11 \mathrm{E}+08$ & 116 \\
\hline 40376 & $5.00 \mathrm{E}+06$ & $1.24 \mathrm{E}+11$ & $6.82 \mathrm{E}+08$ & 182 \\
\hline 70268 & $1.00 \mathrm{E}+07$ & $3.31 \mathrm{E}+11$ & $1.49 \mathrm{E}+09$ & 222 \\
\hline 442903 & $1.00 \mathrm{E}+08$ & $8.57 \mathrm{E}+12$ & $2.02 \mathrm{E}+10$ & 424 \\
\hline 2792858 & $1.00 \mathrm{E}+09$ & $2.22 \mathrm{E}+14$ & $2.73 \mathrm{E}+11$ & 813 \\
\hline
\end{tabular}

Table A.XXI. Absolute Difference from a $10^{-9}$ Solution

\begin{tabular}{|c|c|c|c|}
\hline $\begin{array}{c}\text { Grid } \\
\text { Dimensions }\end{array}$ & $\begin{array}{c}\text { Number of } \\
\text { Fixed Points }\end{array}$ & $\begin{array}{c}\text { Maximum } \\
\text { Absolute } \\
\text { Difference }\end{array}$ & $\begin{array}{c}\text { Average } \\
\text { Absolute } \\
\text { Difference }\end{array}$ \\
\hline 5X5X10 & 18 & 0.0000 & 0.0000 \\
\hline 15X15X30 & 18 & 0.0439 & 0.0130 \\
\hline 15X15X30 & 54 & 0.1329 & 0.0168 \\
\hline 15X15X30 & 250 & 0.1838 & 0.0098 \\
\hline 25X25X50 & 18 & 0.0411 & 0.0104 \\
\hline 25X25X50 & 54 & 0.1489 & 0.0156 \\
\hline 25X25X50 & 250 & 0.1901 & 0.0065 \\
\hline 35X35X70 & 18 & 0.0400 & 0.0089 \\
\hline $35 X 35 X 70$ & 54 & 0.1544 & 0.0139 \\
\hline
\end{tabular}


Table A.XXI. continued

\begin{tabular}{|c|c|c|c|}
\hline $35 \times 35 X 70$ & 250 & 0.1902 & 0.0050 \\
\hline $45 \times 45 \times 90$ & 18 & 0.0387 & 0.0075 \\
\hline $45 \times 45 \times 90$ & 54 & 0.1573 & 0.0125 \\
\hline $45 \times 45 \times 90$ & 250 & 0.1895 & 0.0044 \\
\hline $45 \times 45 \times 90$ & 1026 & 0.0521 & 0.0057 \\
\hline $45 \times 45 \times 90$ & 6750 & 0.0597 & 0.0068 \\
\hline $55 X 55 X 110$ & 18 & 0.0387 & 0.0063 \\
\hline $55 X 55 X 110$ & 54 & 0.1591 & 0.0106 \\
\hline $55 X 55 X 110$ & 250 & 0.1888 & 0.0041 \\
\hline $65 \times 65 X 130$ & 18 & 0.0418 & 0.0056 \\
\hline $65 X 65 X 130$ & 54 & 0.1603 & 0.0099 \\
\hline $65 X 65 X 130$ & 250 & 0.1882 & 0.0040 \\
\hline 75X75150 & 250 & 0.1879 & 0.0042 \\
\hline $75 \times 75 X 150$ & 31250 & 0.0919 & 0.0052 \\
\hline
\end{tabular}

Table A.XXII. Percent Difference from $10^{-9}$ Solution

\begin{tabular}{|c|c|c|c|}
\hline $\begin{array}{c}\text { Grid } \\
\text { Dimensions }\end{array}$ & $\begin{array}{l}\text { Number of } \\
\text { Fixed Points }\end{array}$ & $\begin{array}{c}\text { Maximum \% } \\
\text { Difference }\end{array}$ & $\begin{array}{l}\text { Average \% } \\
\text { Difference }\end{array}$ \\
\hline 5X5X10 & 18 & 0.00 & 0.00 \\
\hline $15 \times 15 X 30$ & 18 & 0.87 & 0.35 \\
\hline 15X15X30 & 54 & 3.24 & 0.50 \\
\hline $15 \times 15 X 30$ & 250 & 4.27 & 0.28 \\
\hline $25 \times 25 \times 50$ & 18 & 0.82 & 0.29 \\
\hline $25 \times 25 X 50$ & 54 & 3.65 & 0.46 \\
\hline $25 \times 25 \times 50$ & 250 & 4.41 & 0.19 \\
\hline $35 \times 35 \times 70$ & 18 & 0.99 & 0.25 \\
\hline $35 \times 35 \times 70$ & 54 & 3.79 & 0.40 \\
\hline $35 \times 35 \times 70$ & 250 & 4.41 & 0.14 \\
\hline $45 \times 45 \times 90$ & 18 & 1.18 & 0.21 \\
\hline $45 \times 45 \times 90$ & 54 & 3.86 & 0.36 \\
\hline $45 \times 45 \times 90$ & 250 & 4.40 & 0.13 \\
\hline $45 \times 45 \times 90$ & 1026 & 0.99 & 0.14 \\
\hline $45 X 45 \times 90$ & 6750 & 1.26 & 0.18 \\
\hline $55 X 55 X 110$ & 18 & 1.32 & 0.18 \\
\hline $55 X 55 X 110$ & 54 & 3.91 & 0.30 \\
\hline 55X55X110 & 250 & 4.38 & 0.12 \\
\hline 65X65X130 & 18 & 1.43 & 0.17 \\
\hline 65X65X130 & 54 & 3.94 & 0.30 \\
\hline 65X65X130 & 250 & 4.37 & 0.11 \\
\hline 75X75X150 & 250 & 4.36 & 0.12 \\
\hline $75 X 75 X 150$ & 31250 & 1.94 & 0.13 \\
\hline
\end{tabular}




\section{APPENDIX B}

This Appendix contains the original regression analysis of the nested-grid results on the solution of the LaPlace equation using SOR and GMRES. It served as the basis for CIPC Paper 2005-112. ${ }^{33}$ Subsequent regression analyses were thought to be preferable and are included in the body of the thesis.

\section{B.1 Laplace SOR: Nested-Grid Method}

Table B.XXIII. Laplace SOR: Fine and Nested-Grid Results

\begin{tabular}{|c|c|c|c|c|c|}
\hline $\mathrm{N}_{\mathrm{FG}}$ & $\mathrm{N}_{\mathrm{CG}}$ & $\omega_{\text {opt }}$ & $\mathrm{N}_{\text {ITER }}$ & TIME(s) & TIME/ITER \\
\hline 250 & 2 & 1.800 & 82 & 0.007 & $8.61 \mathrm{E}-05$ \\
\hline 1458 & 2 & 1.910 & 255 & 0.086 & $3.36 \mathrm{E}-04$ \\
\hline 9826 & 2 & 1.968 & 546 & 1.179 & $2.16 \mathrm{E}-03$ \\
\hline 71874 & 2 & 1.988 & 1104 & 33.562 & $3.04 \mathrm{E}-02$ \\
\hline 549250 & 2 & 1.996 & 3356 & 881.925 & $2.63 \mathrm{E}-01$ \\
\hline 250 & 18 & 1.490 & 32 & 0.003 & $1.41 \mathrm{E}-04$ \\
\hline 1458 & 18 & 1.780 & 58 & 0.019 & $3.24 \mathrm{E}-04$ \\
\hline 9826 & 18 & 1.910 & 182 & 0.410 & $2.19 \mathrm{E}-03$ \\
\hline 71874 & 18 & 1.969 & 483 & 15.328 & $3.26 \mathrm{E}-02$ \\
\hline 549250 & 18 & 1.989 & 902 & 242.160 & $2.68 \mathrm{E}-01$ \\
\hline 1458 & 130 & 1.460 & 37 & 0.019 & $9.54 \mathrm{E}-05$ \\
\hline 9826 & 130 & 1.751 & 79 & 0.191 & $2.42 \mathrm{E}-03$ \\
\hline 71874 & 130 & 1.912 & 212 & 7.081 & $3.40 \mathrm{E}-02$ \\
\hline 549250 & 130 & 1.971 & 533 & 145.910 & $2.74 \mathrm{E}-01$ \\
\hline 9826 & 1026 & 1.410 & 35 & 0.082 & $2.87 \mathrm{E}-03$ \\
\hline 71874 & 1026 & 1.770 & 85 & 2.941 & $3.45 \mathrm{E}-02$ \\
\hline 549250 & 1026 & 1.920 & 257 & 73.582 & $2.92 \mathrm{E}-01$ \\
\hline 71874 & 8194 & 1.430 & 35 & 1.223 & $3.46 \mathrm{E}-02$ \\
\hline 549250 & 8194 & 1.760 & 94 & 28.425 & $2.99 \mathrm{E}-01$ \\
\hline 549250 & 65538 & 1.430 & 37 & 12.160 & $3.28 \mathrm{E}-01$ \\
\hline
\end{tabular}


This data was used to determine the optimal number of fixed-coarse-grid points that should be embedded in a desired fine grid to obtain the pressure solution for any nestedgrid setup in the least amount of time. This was done by regression of the results in Table B.XXIII to obtain mathematical expressions to predict both $\omega_{\text {opt }}$ and the number of iterations $\left(\mathrm{N}_{\text {ITER }}\right)$ required for convergence, for any nested-grid setup. Both of the expressions were functions of the number of fixed-coarse-grid points $\left(\mathrm{N}_{\mathrm{CG}}\right)$ and the total number of fine-grid points $\left(\mathrm{N}_{\mathrm{FG}}\right)$.

The following correlation was found to predict the optimal value of $\omega$ :

$$
\omega_{\text {opt }}=2-\left[2.43 \cdot\left(\mathrm{N}_{\mathrm{CG}}\right)^{0.51} \cdot\left(\mathrm{N}_{\mathrm{FG}}\right)^{-2.40} \cdot\left(\mathrm{N}_{\mathrm{FG}}-\mathrm{N}_{\mathrm{CG}}\right)^{1.88}\right]
$$

It predicts the $\omega_{\mathrm{opt}}$ of Table B.XXIII with a Pearson correlation coefficient of 0.999 or $\mathrm{R}^{2}$ value of 0.998

The expression for the number of iterations required to solve the pressure equations to a tolerance of $10^{-6}$ at $\omega_{\text {opt }}$ is given by the following function:

$$
\mathrm{N}_{\mathrm{ITER}}\left(\mathrm{N}_{\mathrm{CG}}, \mathrm{N}_{\mathrm{FG}}\right)=7.93 \cdot\left(\mathrm{N}_{\mathrm{CG}}\right)^{-0.50} \cdot\left(\mathrm{N}_{\mathrm{FG}}\right)^{0.38} \cdot\left(\mathrm{N}_{\mathrm{FG}}-\mathrm{N}_{\mathrm{CG}}\right)^{0.10}
$$

The correlation coefficient, which correlates the actual and calculated values of $\mathrm{N}_{\text {ITER }}$ for this expression, was 0.995 which is equivalent to a $\mathrm{R}^{2}$ value of 0.990 . 


\section{B.2 GMRES: Nested-Grid Method}

The correlation for the number of iterations as a function of coarse and fine grid points was determined and is as follows

$$
\mathrm{N}_{\mathrm{ITER}}=4.27 \cdot\left(\mathrm{N}_{\mathrm{CG}}\right)^{-0.08} \cdot\left(\mathrm{N}_{\mathrm{FG}}\right)^{-6.06} \cdot\left(\mathrm{N}_{\mathrm{FG}}-\mathrm{N}_{\mathrm{CG}}\right)^{6.40}
$$

The correlation coefficient was 0.984 and the $\mathrm{R}^{2}$ value was 0.969 . Successful application of the nested-grid method was initially expected only for grid sizes equal to or smaller than $17 \times 17 \times 34$.

The results of the fine and nested grid study for GMRES are shown in Table B.XXIV.

Table B.XXIV. Fine and Nested-Grid Results for GMRES

\begin{tabular}{|c|c|c|c|c|}
\hline $\mathrm{N}_{\mathrm{FG}}$ & $\mathrm{N}_{\mathrm{CG}}$ & $\mathrm{N}_{\text {ITER }}$ & TIME(s) & TIME/ITER \\
\hline 250 & 2 & 22 & 0.063 & $2.84 \mathrm{E}-03$ \\
\hline 1458 & 2 & 42 & 0.322 & $7.66 \mathrm{E}-03$ \\
\hline 9826 & 2 & 80 & 3.153 & $3.94 \mathrm{E}-02$ \\
\hline 71874 & 2 & - & - & - \\
\hline 549250 & 2 & - & - & - \\
\hline 250 & 18 & 20 & 0.063 & $3.13 \mathrm{E}-03$ \\
\hline 1458 & 18 & 40 & 0.294 & $7.34 \mathrm{E}-03$ \\
\hline 9826 & 18 & 80 & 4.966 & $6.21 \mathrm{E}-02$ \\
\hline 71874 & 18 & 155 & 355.688 & $2.29 \mathrm{E}+00$ \\
\hline 549250 & 18 & - & - & - \\
\hline 1458 & 130 & 30 & 0.219 & $7.29 \mathrm{E}-03$ \\
\hline 9826 & 130 & 67 & 3.225 & $4.81 \mathrm{E}-02$ \\
\hline 71874 & 130 & 137 & 289.081 & $2.11 \mathrm{E}+00$ \\
\hline 549250 & 130 & - & - & - \\
\hline 9826 & 1026 & 36 & 1.494 & $4.15 \mathrm{E}-02$ \\
\hline 71874 & 1026 & 89 & 123.544 & $1.39 \mathrm{E}+00$ \\
\hline 549250 & 1026 & - & - & - \\
\hline 71874 & 8194 & 36 & 25.534 & $7.09 \mathrm{E}-01$ \\
\hline 549250 & 8194 & - & - & - \\
\hline 549250 & 65538 & - & - & - \\
\hline
\end{tabular}


Table B.XXIV reveals an interesting consequence of imbedding fixed points in the solution method. It can be seen that the original fine-grid solution was only achieved up to 9,826 fine-grid points. Grid sizes above this required too much memory to successfully determine a solution on the desktop computer. As fixed points were added to the fine grid, according to the nested-grid method, the previously unattainable solutions became attainable. The finely-gridded pressures for a grid size of 33x33x66 (71,874 fine grid points) was achieved by applying the nested-grid method using the same desktop computer. This result highlights the potential of the new method to compute reservoir simulation pressures for more refined grids on the same computer. However, even using the nested-grid method, the $65 \times 65 \times 130$ grid was still too large for GMRES to handle on the desktop computer.

\section{B.3 LaPlace SOR: Optimization of the Nested Grid Method}

Simulation runs for various grid sizes at different levels of coarse-grid refinement identified optimal performance of the nested-grid method. A plot of the ratio of time/iteration for all of the nested-grid setups was made as a function of the total grid size and is shown in Figure B.31. The time/iteration was found to vary almost directly with the total number of fine-grid points. This observation was used in the development of a mathematical expression that generates a dimensionless time value which is independent of the computer being used. The dimensionless time expression was in turn used to determine the optimal number of fixed-coarse-grid points to be nested into a given fine grid. The expression for the total dimensionless time was. 


$$
t_{d}=N_{C G} \cdot N_{\text {ITER }}\left(2, N_{C G}\right)+N_{F G} \cdot N_{\text {ITER }}\left(N_{C G}, N_{F G}\right)
$$

\section{Time/Iteration vs Total Grid Size}

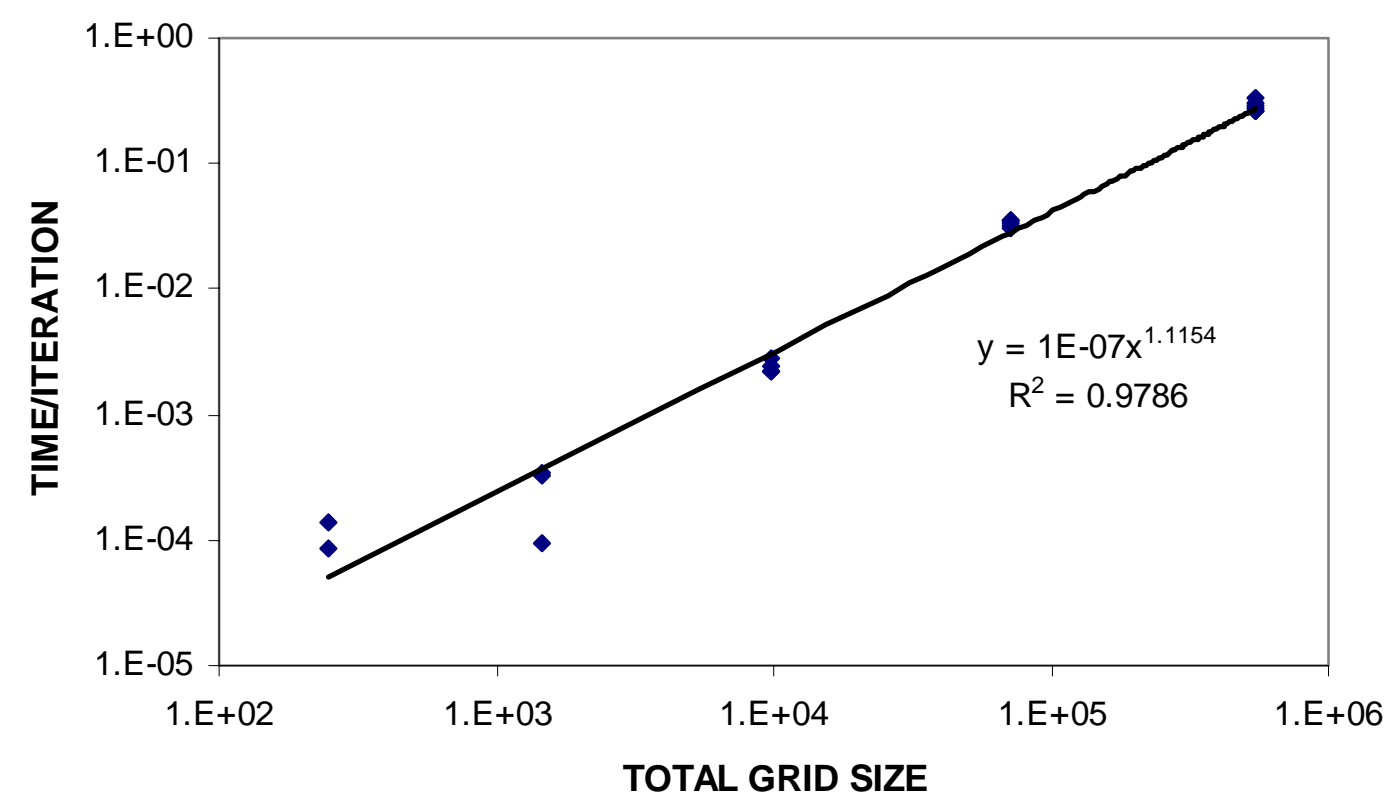

Figure B.31. LaPlace SOR Time/Iteration as a Function of Total Grid

As evident, the dimensionless time expression is made of up two terms: the time required for the calculation of the coarse grid with two wells, and the time required for the calculation of the nested-fine-grid solution with two wells. The number of iterations, $\mathrm{N}_{\text {ITER }}$, is a function of the number of nested-coarse-grid points and the total number of fine-grid points. For the coarse-grid dimensionless-time calculation, the number of iterations is a function of the number of wells (which become nested-coarse-grid points) and the number of coarse-grid points (the number of coarse-grid points is equivalent to the total number of fine-grid points). The time required for the calculation of either the 
coarse or fine-grid pressure solution is the product of the number of coarse or fine grid points used to model the reservoir with the number of iterations required for convergence of the respective grid. The sum of these two yields the total dimensionless time. This function for dimensionless time was optimized by determining the optimum number of fixed-coarse-grid points that should be nested in a given fine-grid solution. The optimization was accomplished using the Solver tool in Microsoft Excel.

The optimized dimensionless time results indicate significant reductions in computational time required to obtain the finely-gridded pressure solution for larger grids. Figure B.32 shows a plot of the improvement obtained by the nested-grid method (SOR_OPTFP) compared to the original full solution without nested-grid pressures (SOR_2FP), both being solved using the SOR algorithm at $\omega_{\text {opt }}$.

\section{Dimensionless Time as a Function of Grid Size}

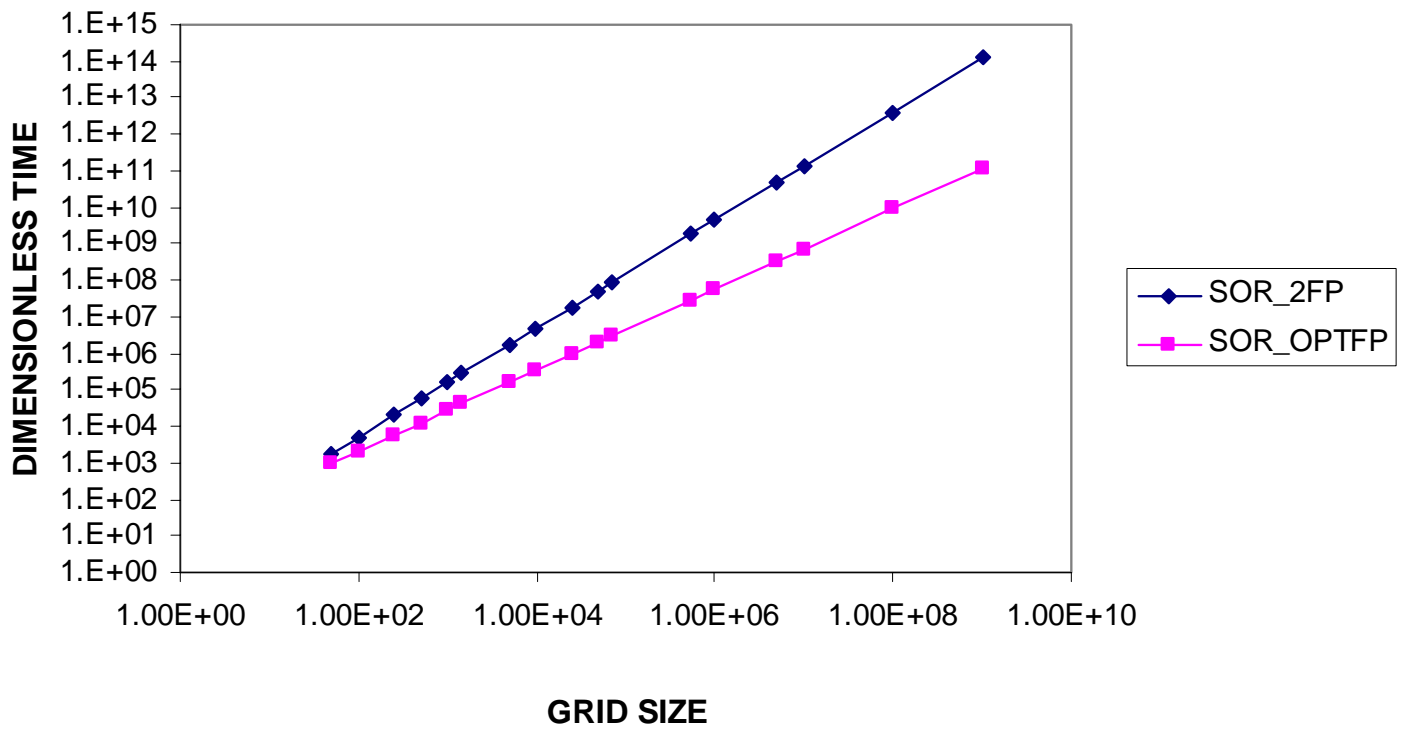

Figure B.32. Improvement Obtained by the Nested-Grid Method 
Table B.XXV summarizes the data displayed in Figure B.32 and shows the ratio of improvement obtained by using the nested-grid method. For a grid size of one million, an improvement of 77 times is noted for the optimized-nested-fine-grid solution over the full solution on a fine grid solved with SOR. Extrapolating the data to one billion points yields an improvement of 1,022 times. This assumes that the computer being used can handle such large memory demands. The desktop used in this study could not.

Table B.XXV. Dimensionless Time Results of Nested-Grid Method

\begin{tabular}{|c|c|c|c|c|}
\hline $\begin{array}{c}\text { OPTIMAL } \\
N_{\mathrm{CG}}\end{array}$ & $\mathrm{N}_{\mathrm{FG}}$ & $\begin{array}{c}\text { FULL } \\
\text { TIME }\end{array}$ & $\begin{array}{c}\text { OPTIMAL } \\
\text { TIME }\end{array}$ & $\begin{array}{c}\text { RATIO OF } \\
\text { IMPROVEMENT }\end{array}$ \\
\hline 13 & $5.00 \mathrm{E}+01$ & $1.84 \mathrm{E}+03$ & $9.51 \mathrm{E}+02$ & 2 \\
\hline 22 & $1.00 \mathrm{E}+02$ & $5.16 \mathrm{E}+03$ & $2.06 \mathrm{E}+03$ & 3 \\
\hline 43 & $2.50 \mathrm{E}+02$ & $2.01 \mathrm{E}+04$ & $5.72 \mathrm{E}+03$ & 4 \\
\hline 72 & $5.00 \mathrm{E}+02$ & $5.62 \mathrm{E}+04$ & $1.24 \mathrm{E}+04$ & 5 \\
\hline 121 & $1.00 \mathrm{E}+03$ & $1.57 \mathrm{E}+05$ & $2.67 \mathrm{E}+04$ & 6 \\
\hline 161 & $1.46 \mathrm{E}+03$ & $2.74 \mathrm{E}+05$ & $4.07 \mathrm{E}+04$ & 7 \\
\hline 403 & $5.00 \mathrm{E}+03$ & $1.71 \mathrm{E}+06$ & $1.60 \mathrm{E}+05$ & 11 \\
\hline 668 & $9.83 \mathrm{E}+03$ & $4.64 \mathrm{E}+06$ & $3.38 \mathrm{E}+05$ & 14 \\
\hline 1341 & $2.50 \mathrm{E}+04$ & $1.85 \mathrm{E}+07$ & $9.53 \mathrm{E}+05$ & 19 \\
\hline 2250 & $5.00 \mathrm{E}+04$ & $5.18 \mathrm{E}+07$ & $2.06 \mathrm{E}+06$ & 25 \\
\hline 2951 & $7.19 \mathrm{E}+04$ & $8.87 \mathrm{E}+07$ & $3.07 \mathrm{E}+06$ & 29 \\
\hline 13484 & $5.49 \mathrm{E}+05$ & $1.81 \mathrm{E}+09$ & $2.93 \mathrm{E}+07$ & 62 \\
\hline 21101 & $1.00 \mathrm{E}+06$ & $4.40 \mathrm{E}+09$ & $5.70 \mathrm{E}+07$ & 77 \\
\hline 70259 & $5.00 \mathrm{E}+06$ & $4.78 \mathrm{E}+10$ & $3.39 \mathrm{E}+08$ & 141 \\
\hline 117953 & $1.00 \mathrm{E}+07$ & $1.33 \mathrm{E}+11$ & $7.31 \mathrm{E}+08$ & 183 \\
\hline 659496 & $1.00 \mathrm{E}+08$ & $4.05 \mathrm{E}+12$ & $9.39 \mathrm{E}+09$ & 432 \\
\hline 3687808 & $1.00 \mathrm{E}+09$ & $1.23 \mathrm{E}+14$ & $1.20 \mathrm{E}+11$ & 1022 \\
\hline
\end{tabular}

A plot of the optimum number of coarse-grid points as a function of the number of fine-grid points is shown in Figure B.33. 


\section{Optimal NCG vs NFG}

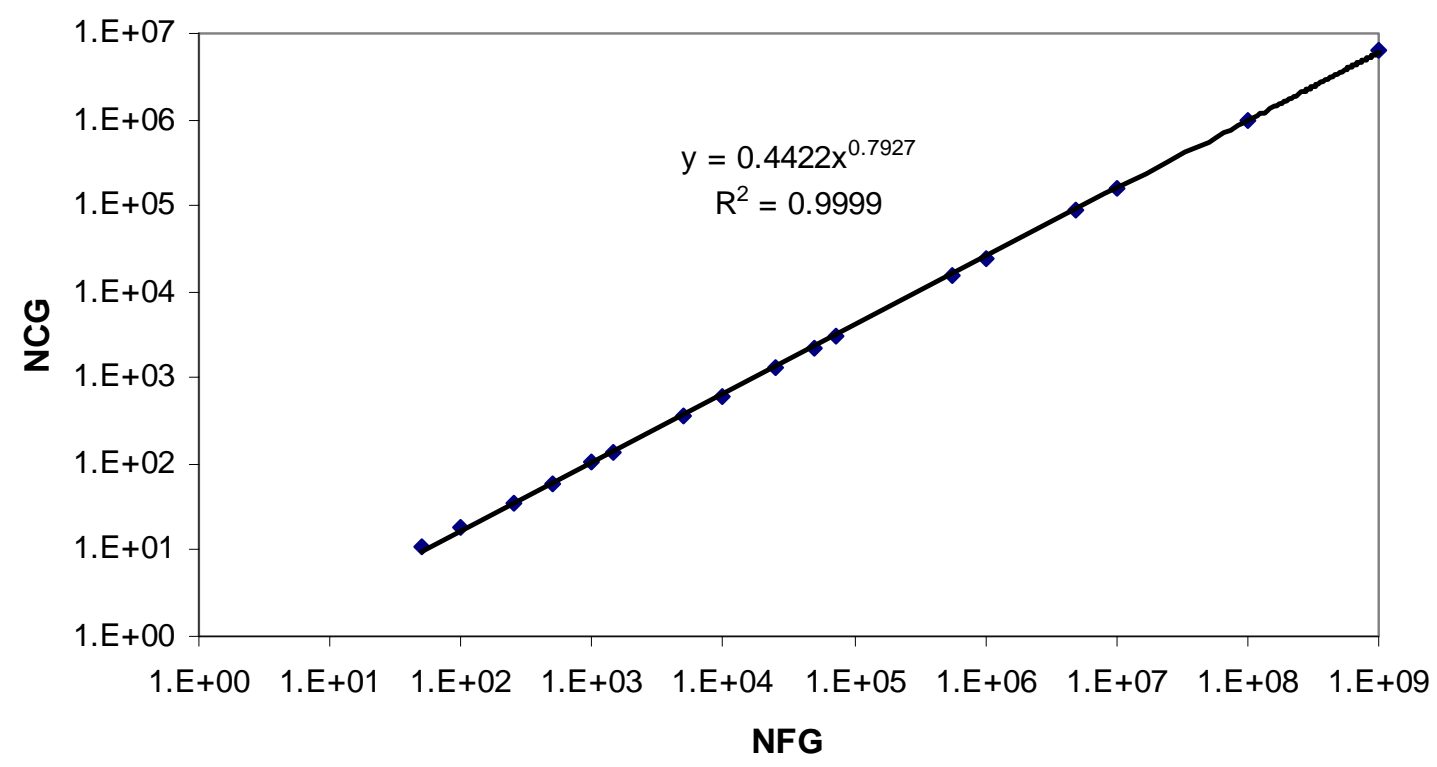

Figure B.33. Optimal Number of Coarse-Grid Points Nested Into Fine Grid

For all of the nested-grid set ups, it was determined that at the optimal number of fixed-coarse-grid points, the calculation of the coarse-grid pressures takes about 20-24\% of the total time, with the nested-fine-grid-calculation taking the remainder of the time. The nested-grid method speeds up the calculation of the finely-gridded reservoir pressures and makes feasible the simulation of larger grids on standard desktop/laptop computers.

\section{B.4 GMRES: Optimization of the Nested Grid Method}

A similar study was done to compare the performance of GMRES with SOR using the new method. A plot of the time/iteration as a function of grid size was also 
constructed from the results obtained from the nested-grid study using GMRES instead of SOR and is shown in Figure B.34.

\section{Time/lteration vs Total Grid Size}

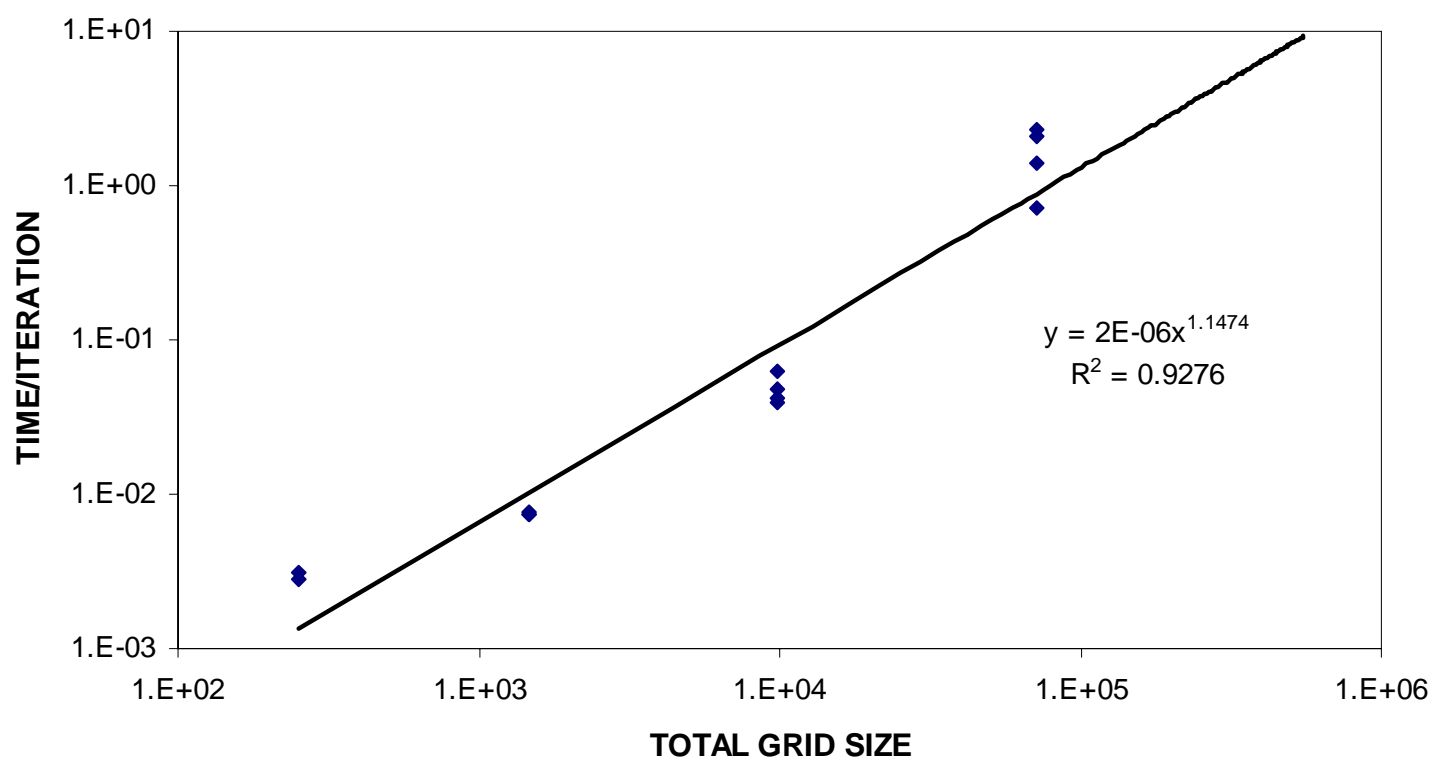

Figure B.34. GMRES: Time/Iteration as a Function of Total Grid Size

The time/iteration was found again to vary more or less directly with the total number of fine-grid points. A dimensionless time analysis was conducted using Equation (B-4) multiplied by twenty to correct the variation in constants. This was done so that SOR and GMRES could be compared directly. The dimensionless times calculated using Equation (B-4) are in essentially the same ratio as the actual time values for the data collected. A correlation coefficient of 0.938 was obtained when the two ratios were analyzed. A plot of the performance, in dimensionless time, of the original GMRES function with the original full fine-grid SOR and SOR applied with the optimized nested-grid method is shown in Figure B.35. These results indicate that the previous conclusion, that GMRES 
would not outperform SOR, is essentially validated except for extremely large grid sizes (1 billion) at which point GMRES has better performance than SOR.

\section{Dimensionless Time as a Function of Grid Size}

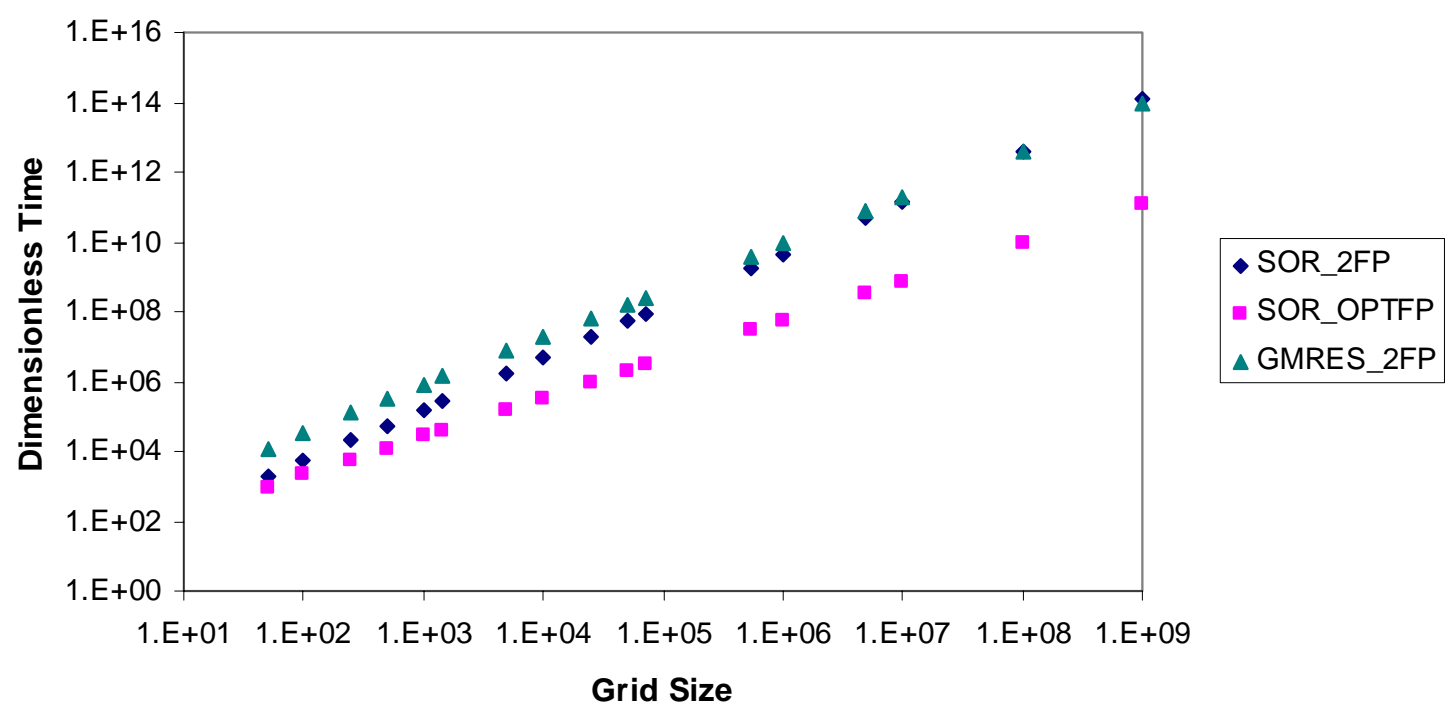

Figure B.35. Optimized Nested-Grid Method Using SOR Compared with GMRES

Determination of the optimal number of fixed-grid points that should be nested into the fine grid using GMRES was determined next. The results are displayed in Table B.XXVI.

Table B.XXVI. Dimensionless Time Results of Nested-Grid Method

\begin{tabular}{|c|c|c|c|c|}
\hline $\begin{array}{c}\text { OPTIMAL } \\
\mathrm{N}_{\mathrm{CG}}\end{array}$ & $\mathrm{N}_{\mathrm{FG}}$ & $\begin{array}{c}\text { FULL } \\
\text { TIME }\end{array}$ & $\begin{array}{c}\text { OPTIMAL } \\
\text { TIME }\end{array}$ & $\begin{array}{c}\text { RATIO OF } \\
\text { IMPROVEMENT }\end{array}$ \\
\hline 16 & $5.00 \mathrm{E}+01$ & $1.17 \mathrm{E}+04$ & $2.51 \mathrm{E}+03$ & 4.7 \\
\hline 30 & $1.00 \mathrm{E}+02$ & $3.39 \mathrm{E}+04$ & $8.14 \mathrm{E}+03$ & 4.2 \\
\hline 71 & $2.50 \mathrm{E}+02$ & $1.25 \mathrm{E}+05$ & $3.21 \mathrm{E}+04$ & 3.9 \\
\hline 137 & $5.00 \mathrm{E}+02$ & $3.24 \mathrm{E}+05$ & $8.45 \mathrm{E}+04$ & 3.8 \\
\hline 268 & $1.00 \mathrm{E}+03$ & $8.31 \mathrm{E}+05$ & $2.16 \mathrm{E}+05$ & 3.8 \\
\hline 385 & $1.46 \mathrm{E}+03$ & $1.38 \mathrm{E}+06$ & $3.58 \mathrm{E}+05$ & 3.9 \\
\hline 1266 & $5.00 \mathrm{E}+03$ & $7.24 \mathrm{E}+06$ & $1.83 \mathrm{E}+06$ & 4.0 \\
\hline 2430 & $9.83 \mathrm{E}+03$ & $1.79 \mathrm{E}+07$ & $4.44 \mathrm{E}+06$ & 4.0 \\
\hline 5984 & $2.50 \mathrm{E}+04$ & $6.26 \mathrm{E}+07$ & $1.51 \mathrm{E}+07$ & 4.1 \\
\hline
\end{tabular}


Table B.XXVI. continued

\begin{tabular}{|c|c|c|c|c|}
\hline 11676 & $5.00 \mathrm{E}+04$ & $1.58 \mathrm{E}+08$ & $3.74 \mathrm{E}+07$ & 4.2 \\
\hline 16564 & $7.19 \mathrm{E}+04$ & $2.57 \mathrm{E}+08$ & $6.02 \mathrm{E}+07$ & 4.3 \\
\hline 117187 & $5.49 \mathrm{E}+05$ & $3.92 \mathrm{E}+09$ & $8.59 \mathrm{E}+08$ & 4.6 \\
\hline 208339 & $1.00 \mathrm{E}+06$ & $8.74 \mathrm{E}+09$ & $1.88 \mathrm{E}+09$ & 4.6 \\
\hline 974551 & $5.00 \mathrm{E}+06$ & $7.54 \mathrm{E}+10$ & $1.54 \mathrm{E}+10$ & 4.9 \\
\hline 1891579 & $1.00 \mathrm{E}+07$ & $1.91 \mathrm{E}+11$ & $3.79 \mathrm{E}+10$ & 5.0 \\
\hline 17024133 & $1.00 \mathrm{E}+08$ & $4.16 \mathrm{E}+12$ & $7.61 \mathrm{E}+11$ & 5.5 \\
\hline 151734513 & $1.00 \mathrm{E}+09$ & $9.07 \mathrm{E}+13$ & $1.52 \mathrm{E}+13$ & 6.0 \\
\hline
\end{tabular}

These results show an average speed up of about 4.5 times in the performance of GMRES when the optimal number of fixed points is nested into the solution algorithm. It is evident that GMRES by itself does not perform as well as SOR; this was observed in the initial study of GMRES. The optimized performance of GMRES is seen to be much slower than the optimized performance of SOR especially at larger grid sizes; however the optimized performance of GMRES does outperform SOR_2FP at larger grid sizes. Figure B.36 displays the results.

These results indicate that SOR generates significant improvement over even advanced algorithms such as GMRES when the new method is applied. It is believed that improved performance of the pressure solution calculation with the nested-grid method using SOR is due to the increased number of fixed points around which the final solution can relax. 


\section{Dimensionless Time as a Function of Grid Size}

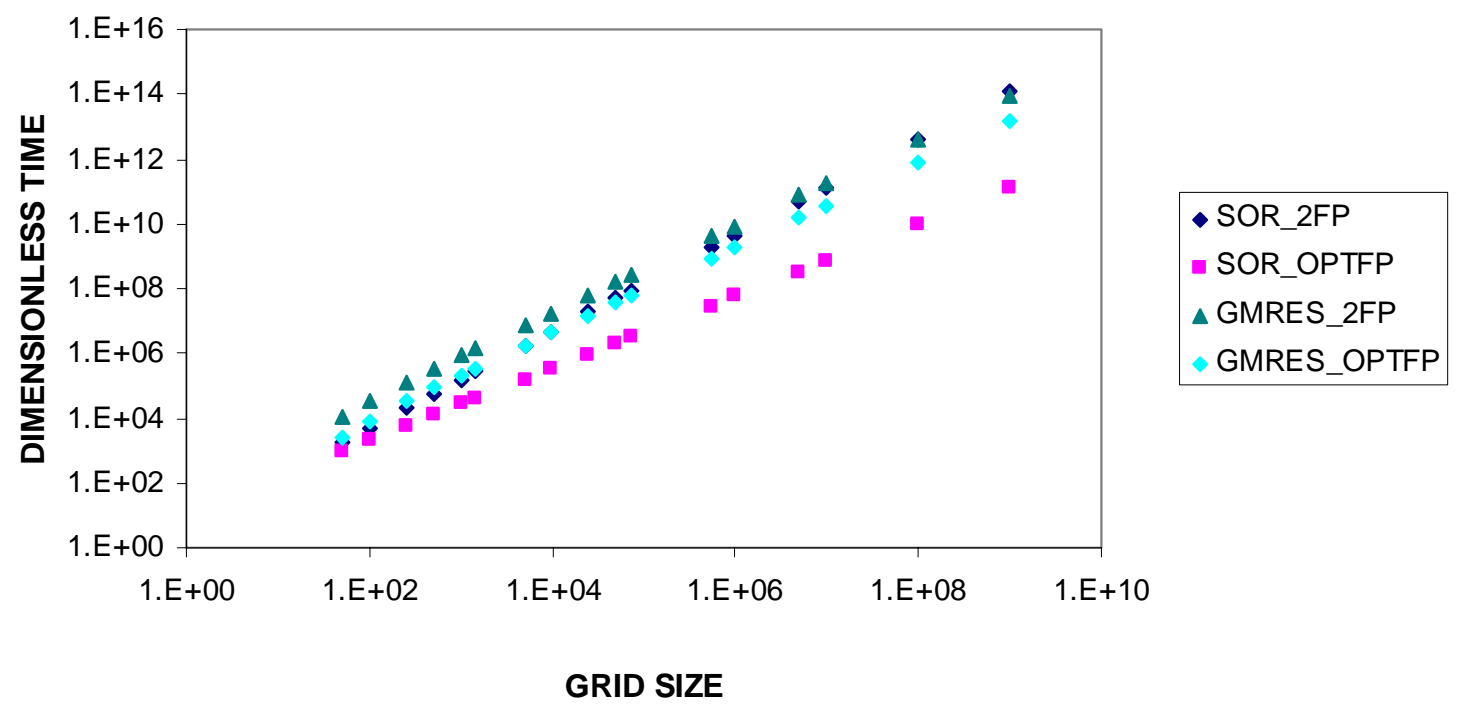

Figure B.36. Comparison of Improvement Obtained by the Nested-Grid Method for GMRES and SOR 


\section{APPENDIX C}

This Appendix contains the original regression analysis of the nested-grid results on the solution of the pressure using Weber's coefficients. Subsequent regression analyses were thought to be preferable and are included in the body of the thesis.

\section{C.1 Weber SOR: Nested-Grid Results}

Table C.XXVII. Weber SOR: Fine and Nested-Grid Results with Weber’s Coefficients

\begin{tabular}{|c|c|c|c|c|c|c|}
\hline DIMENSIONS & $\mathrm{N}_{\mathrm{FG}}$ & $\mathrm{N}_{\mathrm{CG}}$ & $\begin{array}{c}\text { OPT } \\
\text { ORF }\end{array}$ & NITER & TIME(s) & TIME/ITER \\
\hline $5 \times 5 \times 10$ & 250 & 2 & 1.9831 & 634 & 0.049 & $7.80 \mathrm{E}-05$ \\
\hline $15 \times 15 \times 30$ & 6750 & 2 & 1.9947 & 1875 & 4.489 & $2.39 \mathrm{E}-03$ \\
\hline $25 \times 25 \times 50$ & 31250 & 2 & 1.9975 & 3410 & 124.852 & $3.66 \mathrm{E}-02$ \\
\hline $35 \times 35 \times 70$ & 85750 & 2 & 1.9977 & 4762 & 545.705 & $1.15 \mathrm{E}-01$ \\
\hline $45 \times 45 \times 90$ & 182250 & 2 & 1.9982 & 6370 & 1640.267 & $2.57 \mathrm{E}-01$ \\
\hline $55 \times 55 \times 110$ & 332750 & 2 & 1.9985 & 8284 & 3785.215 & $4.57 \mathrm{E}-01$ \\
\hline $65 \times 65 \times 130$ & 549250 & 2 & 1.9989 & 10371 & 8036.465 & $7.75 \mathrm{E}-01$ \\
\hline $75 \times 75 \times 150$ & 843750 & 2 & 1.999 & 13057 & 15704.155 & $1.20 \mathrm{E}+00$ \\
\hline $5 \times 5 \times 10$ & 250 & 16 & 1.47 & 25 & 0.002 & $7.50 \mathrm{E}-05$ \\
\hline $15 \times 15 \times 30$ & 6750 & 16 & 1.89 & 115 & 1.372 & $1.19 \mathrm{E}-02$ \\
\hline $25 \times 25 \times 50$ & 31250 & 16 & 1.96 & 250 & 15.762 & $6.30 \mathrm{E}-02$ \\
\hline $35 \times 35 \times 70$ & 85750 & 16 & 1.974 & 361 & 65.910 & $1.83 \mathrm{E}-01$ \\
\hline $45 \times 45 \times 90$ & 182250 & 16 & 1.9795 & 470 & 116.330 & $2.48 \mathrm{E}-01$ \\
\hline $55 \times 55 \times 110$ & 332750 & 16 & 1.9851 & 584 & 266.795 & $4.57 \mathrm{E}-01$ \\
\hline $65 \times 65 \times 130$ & 549250 & 16 & 1.9888 & 536 & 432.823 & $8.08 \mathrm{E}-01$ \\
\hline $75 \times 75 \times 150$ & 843750 & 16 & 1.9912 & 1209 & 1534.991 & $1.27 \mathrm{E}+00$ \\
\hline $15 \times 15 \times 30$ & 6750 & 54 & 1.81 & 75 & 1.040 & $1.39 \mathrm{E}-02$ \\
\hline $25 \times 25 \times 50$ & 31250 & 54 & 1.911 & 142 & 9.063 & $6.38 \mathrm{E}-02$ \\
\hline $35 \times 35 \times 70$ & 85750 & 54 & 1.951 & 214 & 39.997 & $1.87 \mathrm{E}-01$ \\
\hline $45 \times 45 \times 90$ & 182250 & 54 & 1.963 & 315 & 79.660 & $2.53 \mathrm{E}-01$ \\
\hline $55 \times 55 \times 110$ & 332750 & 54 & 1.974 & 340 & 158.568 & $4.66 \mathrm{E}-01$ \\
\hline $65 \times 65 \times 130$ & 549250 & 54 & 1.981 & 263 & 211.304 & $8.03 \mathrm{E}-01$ \\
\hline
\end{tabular}


Table C.XXVII. continued

\begin{tabular}{|c|c|c|c|c|c|c|}
\hline $75 \times 75 \times 150$ & 843750 & 54 & & & & \\
\hline $15 \times 15 \times 30$ & 6750 & 128 & 1.75 & 55 & 0.126 & $2.29 \mathrm{E}-03$ \\
\hline $45 \times 45 \times 90$ & 182250 & 128 & 1.94 & 254 & 64.549 & $2.54 \mathrm{E}-01$ \\
\hline $75 \times 75 \times 150$ & 843750 & 128 & & & & \\
\hline $15 \times 15 \times 30$ & 6750 & 250 & 1.678 & 41 & 0.660 & $1.61 \mathrm{E}-02$ \\
\hline $25 \times 25 \times 50$ & 31250 & 250 & 1.829 & 94 & 6.262 & $6.66 \mathrm{E}-02$ \\
\hline $35 \times 35 \times 70$ & 85750 & 250 & 1.883 & 128 & 24.113 & $1.88 \mathrm{E}-01$ \\
\hline $45 \times 45 \times 90$ & 182250 & 250 & 1.9159 & 183 & 47.596 & $2.60 \mathrm{E}-01$ \\
\hline $55 \times 55 \times 110$ & 332750 & 250 & 1.9513 & 277 & 132.412 & $4.78 \mathrm{E}-01$ \\
\hline $65 \times 65 \times 130$ & 549250 & 250 & 1.9529 & 289 & 237.171 & $8.21 \mathrm{E}-01$ \\
\hline $75 \times 75 \times 150$ & 843750 & 250 & 1.9617 & 347 & 436.920 & $1.26 \mathrm{E}+00$ \\
\hline $45 \times 45 \times 90$ & 182250 & 1024 & 1.817 & 123 & 31.412 & $2.55 \mathrm{E}-01$ \\
\hline $75 \times 75 \times 150$ & 843750 & 1024 & & & & \\
\hline $45 \times 45 \times 90$ & 182250 & 6750 & 1.63 & 48 & 12.238 & $2.55 \mathrm{E}-01$ \\
\hline $75 \times 75 \times 150$ & 843750 & 6750 & & & & \\
\hline $75 \times 75 \times 150$ & 843750 & 31250 & 1.631 & 48 & 60.624 & $1.26 \mathrm{E}+00$ \\
\hline
\end{tabular}

A regression of the results in Table C.XXVII allowed the generation of mathematical expressions to predict both $\omega_{\text {opt }}$ and $\mathrm{N}_{\text {ITER }}$ required for convergence for any nested-grid setup. The following correlation predicts the optimal value of the overrelaxation factor $\omega_{\text {opt }}$ with a correlation coefficient of 0.973 or a $\mathrm{R}^{2}$ value of 0.94 .

$$
\omega_{\text {opt }}=2-\left[1.99 \cdot\left(\mathrm{N}_{\mathrm{CG}}\right)^{0.54} \cdot\left(\mathrm{N}_{\mathrm{FG}}\right)^{-0.53} \cdot\left(\mathrm{N}_{\mathrm{FG}}-\mathrm{N}_{\mathrm{CG}}\right)^{0.00}\right]
$$

The mathematical expression for the number of iterations required to reach a convergence criterion of $10^{-6}$ had a correlation coefficient of 0.997 or a $\mathrm{R}^{2}$ value of 0.995 . Once again the convergence criterion was the absolute difference between the two entirely opposite grid cells $\mathrm{P}_{1,1,1}$ and $\mathrm{P}_{\mathrm{Imax}, J \max , K \operatorname{Kmax}}$.

$$
\mathrm{N}_{\mathrm{ITER}}=87.50 \cdot\left(\mathrm{N}_{\mathrm{CG}}\right)^{-1.21} \cdot\left(\mathrm{N}_{\mathrm{FG}}\right)^{0.41} \cdot\left(\mathrm{N}_{\mathrm{FG}}-\mathrm{N}_{\mathrm{CG}}\right)^{0.02}
$$




\section{C.2 Optimization of the Nested Grid Method}

A plot of the ratio of time/iteration as a function of total grid size was constructed and is shown in Figure C.37.

\section{Time/lteration vs Total Grid Size}

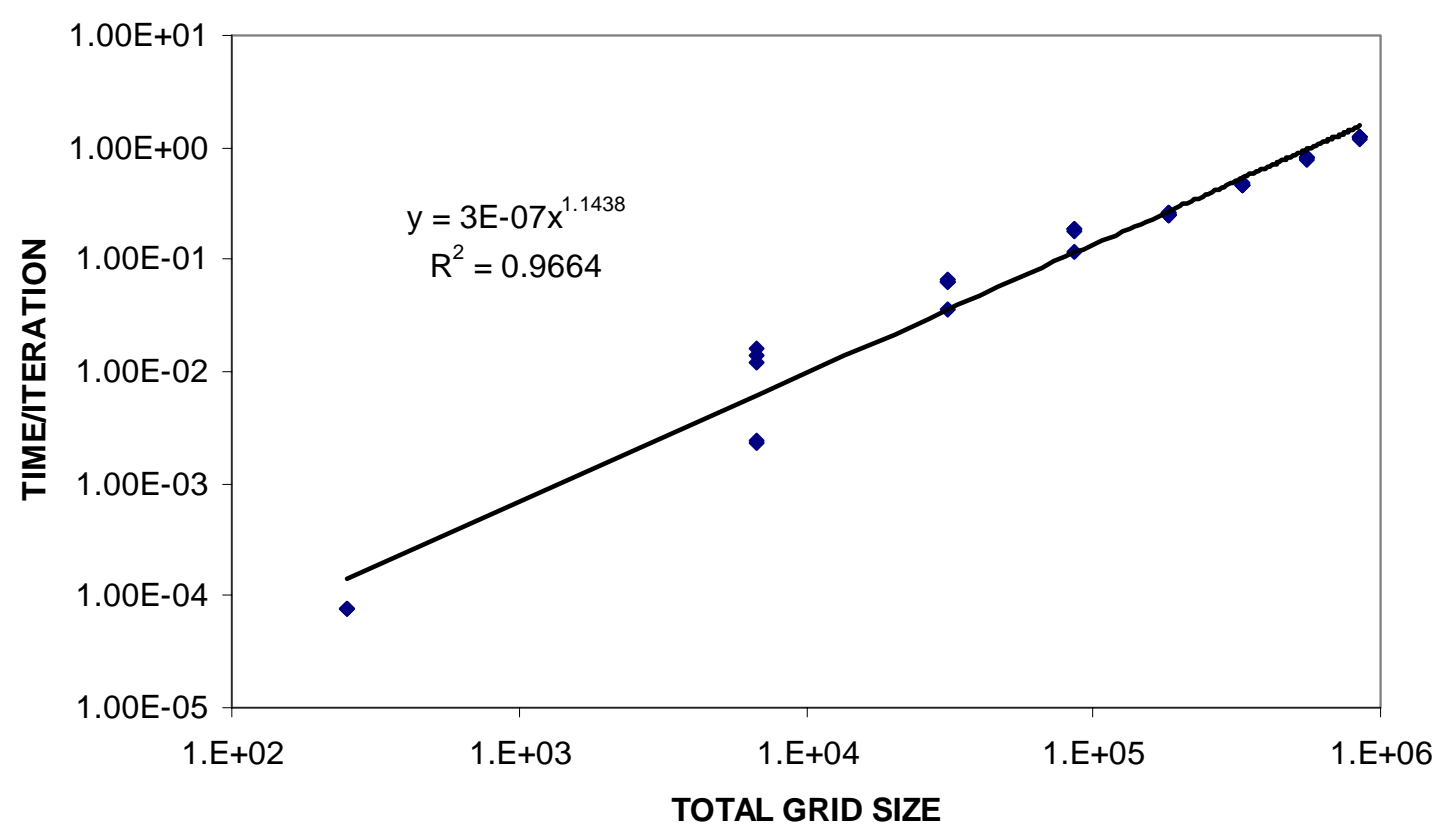

Figure C.37. Weber Time/Iteration as a Function of Total Grid Size

Once again the time/iteration was found to vary almost directly with the total number of fine-grid points. The same expression for dimensionless time was used as explained previously in Appendix B.

$$
t_{d}=N_{C G} \cdot N_{\text {ITER }}\left(2, N_{C G}\right)+N_{F G} \cdot N_{I T E R}\left(N_{C G}, N_{F G}\right)
$$


A comparison of the dimensionless time required to achieve a fine-grid solution without fixed points and with the optimal number of fixed points nested into the fine grid showed a dramatic enhancement in performance.

\section{Dimensionless Time as a Function of Grid Size:Weber's Equations}

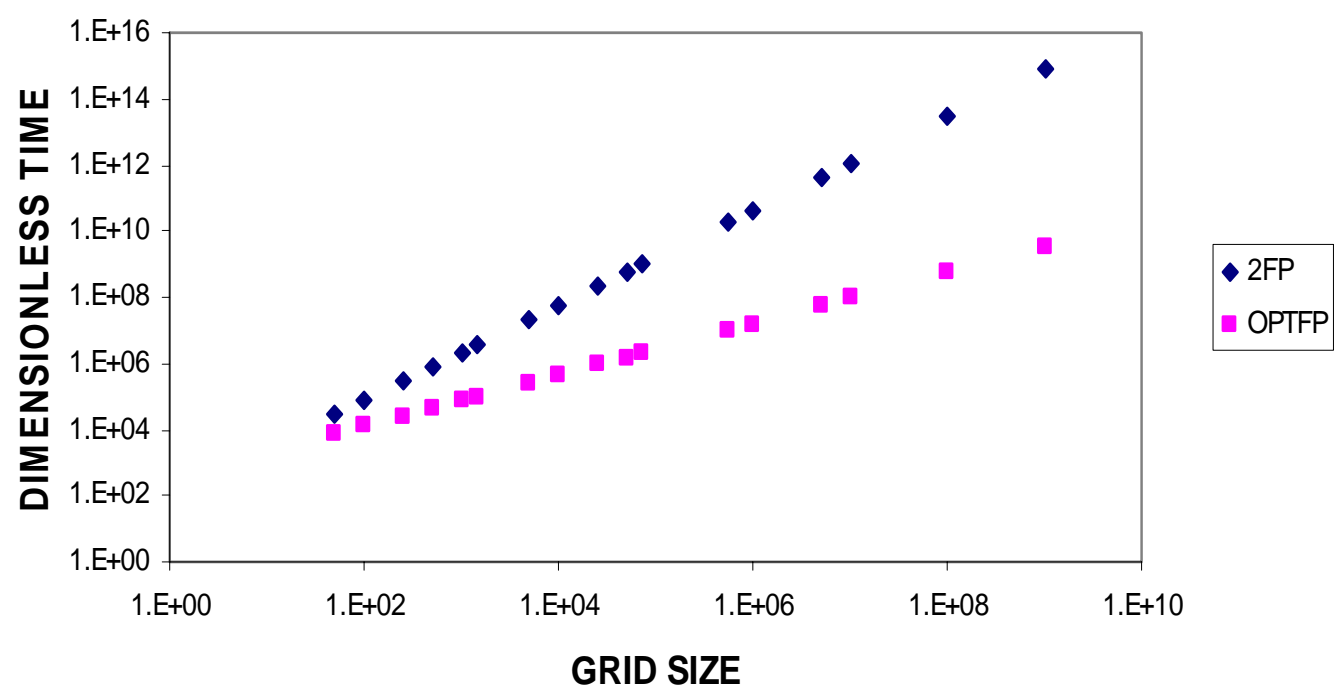

Figure C.38. Improvement Obtained by Nested-Grid Method

Table C.XXVIII shows the data displayed in Figure C.38 as well as the ratio of improvement obtained by using the nested-grid method. For a fine grid size of one million, the ratio of improvement of dimensionless time required for the original fine-grid solution to the fine-grid solution obtained using the optimum number of nested fixed points is 2,659. This is a tremendous increase. Comparing the performance of the nestedgrid method using Weber's coefficients with the work done previously which used traditional finite-difference equations and GMRES, shows that Weber's coefficients enhance the nested-grid performance even further for large grids. 
Table C.XXVIII. Performance of Nested-Grid Method Using Weber's Coefficients

\begin{tabular}{|c|c|c|c|c|}
\hline $\begin{array}{c}\text { OPTIMAL } \\
\mathrm{N}_{\mathrm{CG}}\end{array}$ & $\mathrm{N}_{\mathrm{FG}}$ & FULL TIME & $\begin{array}{c}\text { OPTIMAL } \\
\text { TIME }\end{array}$ & $\begin{array}{c}\text { RATIO OF } \\
\text { IMPROVEMENT }\end{array}$ \\
\hline 11 & $5.00 \mathrm{E}+01$ & $3.01 \mathrm{E}+04$ & $7.30 \mathrm{E}+03$ & 4 \\
\hline 16 & $1.00 \mathrm{E}+02$ & $8.09 \mathrm{E}+04$ & $1.25 \mathrm{E}+04$ & 6 \\
\hline 26 & $2.50 \mathrm{E}+02$ & $2.99 \mathrm{E}+05$ & $2.54 \mathrm{E}+04$ & 12 \\
\hline 37 & $5.00 \mathrm{E}+02$ & $8.03 \mathrm{E}+05$ & $4.33 \mathrm{E}+04$ & 19 \\
\hline 54 & $1.00 \mathrm{E}+03$ & $2.16 \mathrm{E}+06$ & $7.40 \mathrm{E}+04$ & 29 \\
\hline 67 & $1.46 \mathrm{E}+03$ & $3.69 \mathrm{E}+06$ & $9.91 \mathrm{E}+04$ & 37 \\
\hline 130 & $5.00 \mathrm{E}+03$ & $2.14 \mathrm{E}+07$ & $2.57 \mathrm{E}+05$ & 83 \\
\hline 188 & $9.83 \mathrm{E}+03$ & $5.61 \mathrm{E}+07$ & $4.33 \mathrm{E}+05$ & 130 \\
\hline 311 & $2.50 \mathrm{E}+04$ & $2.13 \mathrm{E}+08$ & $8.91 \mathrm{E}+05$ & 239 \\
\hline 453 & $5.00 \mathrm{E}+04$ & $5.71 \mathrm{E}+08$ & $1.52 \mathrm{E}+06$ & 375 \\
\hline 551 & $7.19 \mathrm{E}+04$ & $9.58 \mathrm{E}+08$ & $2.01 \mathrm{E}+06$ & 476 \\
\hline 1659 & $5.49 \mathrm{E}+05$ & $1.74 \mathrm{E}+10$ & $9.69 \mathrm{E}+06$ & 1797 \\
\hline 2295 & $1.00 \mathrm{E}+06$ & $4.09 \mathrm{E}+10$ & $1.54 \mathrm{E}+07$ & 2659 \\
\hline 5488 & $5.00 \mathrm{E}+06$ & $4.06 \mathrm{E}+11$ & $5.34 \mathrm{E}+07$ & 7611 \\
\hline 7990 & $1.00 \mathrm{E}+07$ & $1.09 \mathrm{E}+12$ & $9.12 \mathrm{E}+07$ & 11971 \\
\hline 27814 & $1.00 \mathrm{E}+08$ & $2.91 \mathrm{E}+13$ & $5.40 \mathrm{E}+08$ & 53903 \\
\hline 96830 & $1.00 \mathrm{E}+09$ & $7.76 \mathrm{E}+14$ & $3.20 \mathrm{E}+09$ & 242710 \\
\hline
\end{tabular}

\section{Dimensionless Time as a Function of Grid Size}

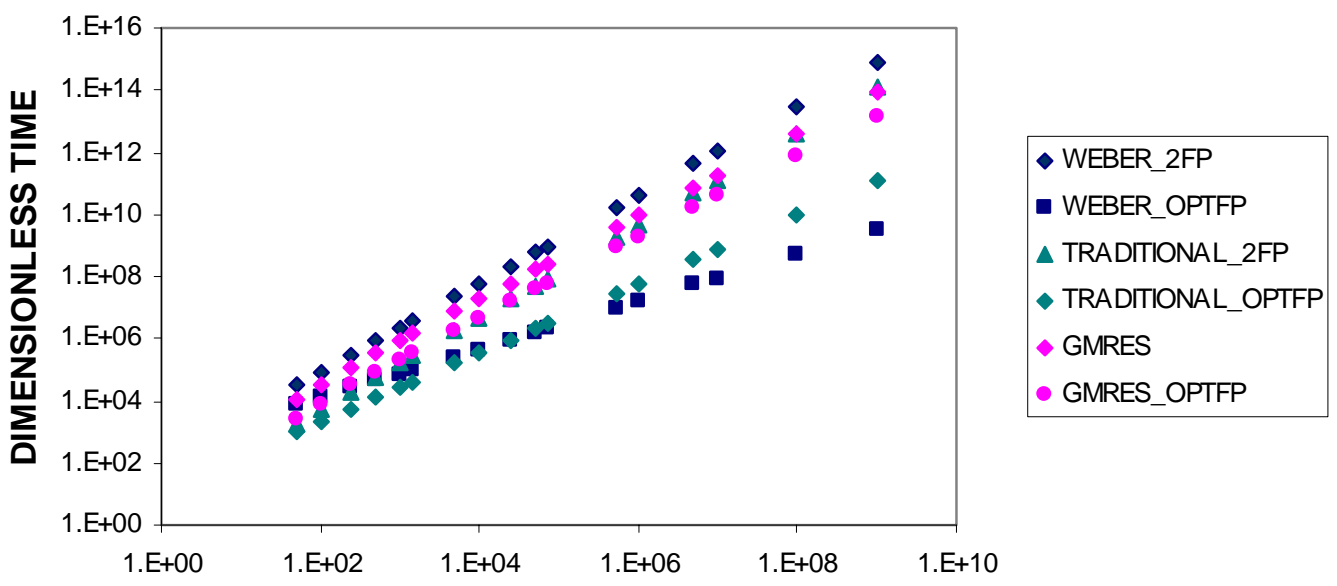

GRID SIZE

Figure C.39. Comparison of Dimensionless Time for all Solution Algorithms 


\section{APPENDIX D \\ OPTIMAL OVER-RELAXATION FACTOR FINDER}

The implementation of the nested-grid method, using Successive-Over-Relaxation, requires that an optimal value be used for the over-relaxation factor in order to achieve best performance. In the previous portions of the study, many hours were spent running the pressure simulations at different over-relaxation factors to determine which was best suited for the particular grid system being studied at the time. This quickly became a time consuming but necessary part of the project. In order to make the method of SuccessiveOver-Relaxation more suitable for real-life implementation in the nested-grid method, it was hoped that an algorithm could be developed that would allow the determination of the optimal over-relaxation factor, $\omega_{\text {opt, }}$ while the interative solution progressed.

The residual or error in the calculated pressure from its final value was observed to decay exponentially; consequently, an exponential decay term was used in an equation which approximated the pressure at a given iteration $n$ in the relaxation process.

$$
\mathrm{P}^{\mathrm{n}}=\mathrm{P}^{\infty}+\mathrm{ae}^{-\mathrm{bn}}
$$

$P$ is the pressure at grid point $\mathrm{P}_{1,1,1}$ or $\mathrm{P}_{\operatorname{Imax}, \mathrm{Jmax}, \mathrm{Kmax}}, \mathrm{P}^{\infty}$ is the pressure at the same grid point after an infinite number of iterations, and the $a e^{-b n}$ term represents the error in the 
pressure solution which decays exponentially. This equation was used at three different iteration steps to determine how the $b$ value changed throughout the convergence process at different over-relaxation factors. In equation (D-1) the values of $P$ can be the pressure at any particular point, yet the convergence criterion in this study was the absolute difference between $\mathrm{P}_{1,1,1}$ and $\mathrm{P}_{\text {Imax,JImax,Kmax }}$. Consequently, for the following analysis, the values of $P$ are the value of the absolute difference between these two grid points as the solution progresses to convergence. The following equations show how the value of $b$ was determined. Initially equation (D-1) was written for three different iteration steps as follows.

$$
\begin{aligned}
\mathrm{P}^{\mathrm{n}} & =\mathrm{P}^{\infty}+\mathrm{ae}^{-\mathrm{bn}} \\
\mathrm{P}^{\mathrm{n}-1} & =\mathrm{P}^{\infty}+\mathrm{ae}^{-\mathrm{b}(\mathrm{n}-1)} \\
\mathrm{P}^{\mathrm{n}-2} & =\mathrm{P}^{\infty}+\mathrm{ae}^{-\mathrm{b}(\mathrm{n}-2)}
\end{aligned}
$$

These equations were subtracted from each other which makes the $P^{\infty}$ term drop out.

$$
\begin{gathered}
\mathrm{P}^{\mathrm{n}}-\mathrm{P}^{\mathrm{n}-1}=a e^{-\mathrm{bn}}-\mathrm{ae}^{-\mathrm{b}(\mathrm{n}-1)}=\mathrm{ae}^{-\mathrm{bn}}\left(1-\mathrm{e}^{\mathrm{b}}\right) \\
\mathrm{P}^{\mathrm{n}-1}-\mathrm{P}^{\mathrm{n}-2}=a \mathrm{e}^{-\mathrm{b}(\mathrm{n}-1)}-\mathrm{a} \mathrm{e}^{-\mathrm{b}(\mathrm{n}-2)}=\mathrm{ae}^{-\mathrm{b}(\mathrm{n}-1)}\left(1-\mathrm{e}^{\mathrm{b}}\right)
\end{gathered}
$$

The ratio of the differences was calculated which eliminates the $a$ variable

$$
\frac{\mathrm{P}^{\mathrm{n}}-\mathrm{P}^{\mathrm{n}-1}}{\mathrm{P}^{\mathrm{n}-1}-\mathrm{P}^{\mathrm{n}-2}}=\frac{\mathrm{e}^{-\mathrm{bn}}}{\mathrm{e}^{-\mathrm{b}(\mathrm{n}-1)}}=\frac{\mathrm{e}^{-\mathrm{bn}}}{\mathrm{e}^{-\mathrm{bn}} \mathrm{e}^{\mathrm{b}}}=\mathrm{e}^{-\mathrm{b}}
$$


The resulting equation is solved for $b$.

$$
\mathrm{b}=-\ln \frac{\mathrm{P}^{\mathrm{n}}-\mathrm{P}^{\mathrm{n}-1}}{\mathrm{P}^{\mathrm{n}-1}-\mathrm{P}^{\mathrm{n}-2}}
$$

Equation (D-8) is the mathematical expression that determined how the value of $b$ changed with respect to the iteration process at a particular over-relaxation factor. It was assumed initially that a large $b$ value would be evident as the optimal over-relaxation factor was approached, indicating an increased rate of convergence. If this were the case, an algorithm to determine $\omega_{\text {opt }}$ would simply have to adjust the over-relaxation factor during the iteration process until the largest value of $b$ was calculated and then finish relaxing to the solution at the over-relaxation factor that generated the largest $b$ value.

A number of pressure simulations were run in order to determine if the $b$ value changed in a distinct manner as the optimal over-relaxation factor was approached. The following figures summarize the results obtained for a $15 \times 15 \times 30$ grid with 250 nestedgrid points fixed in the solution method using Weber's equations. As the $b$ value required a minimum of three iterations to be determined, the plots' independent variable,Number of B Values, is the number of iterations required for convergence minus two. The dependent variable is simply the value of $b$ as expressed in equation (D-8). 
ORF 1.0

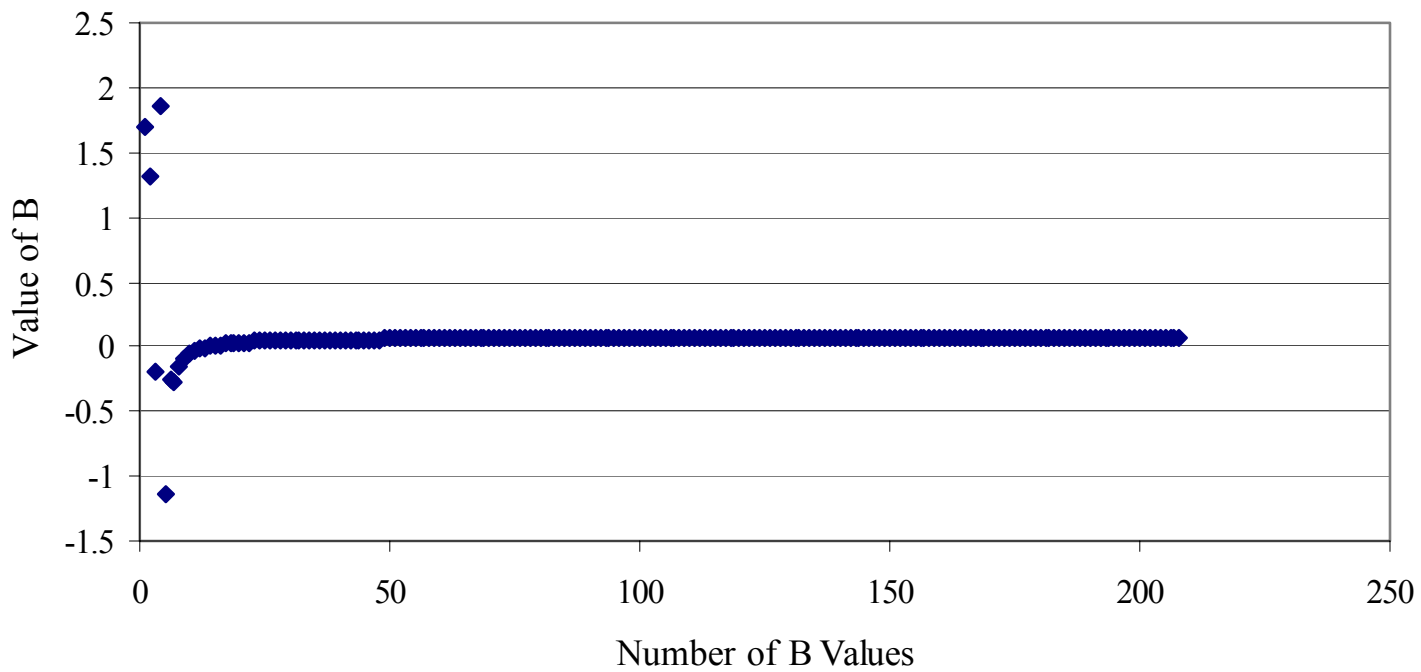

Figure D.40. ORF 1.0

ORF 1.1

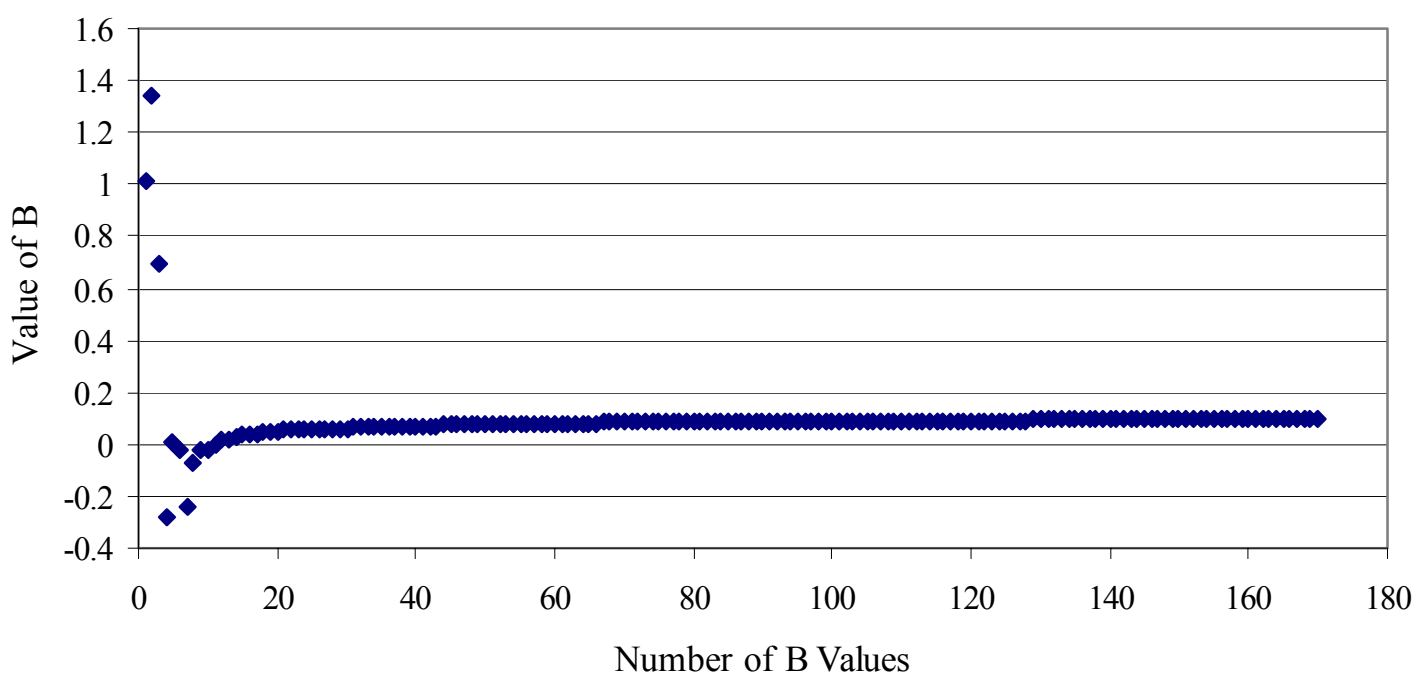

Figure D.41. ORF 1.1 
ORF 1.2

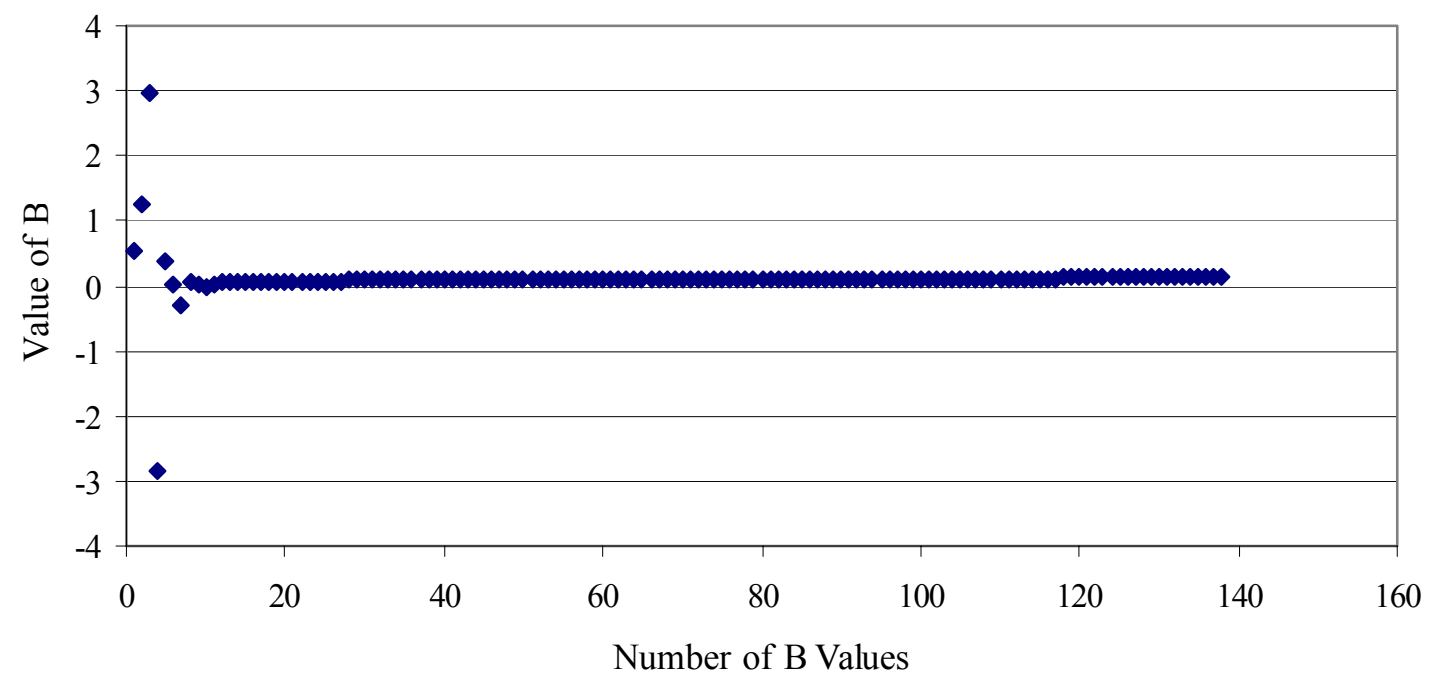

Figure D.42. ORF 1.2

ORF 1.3

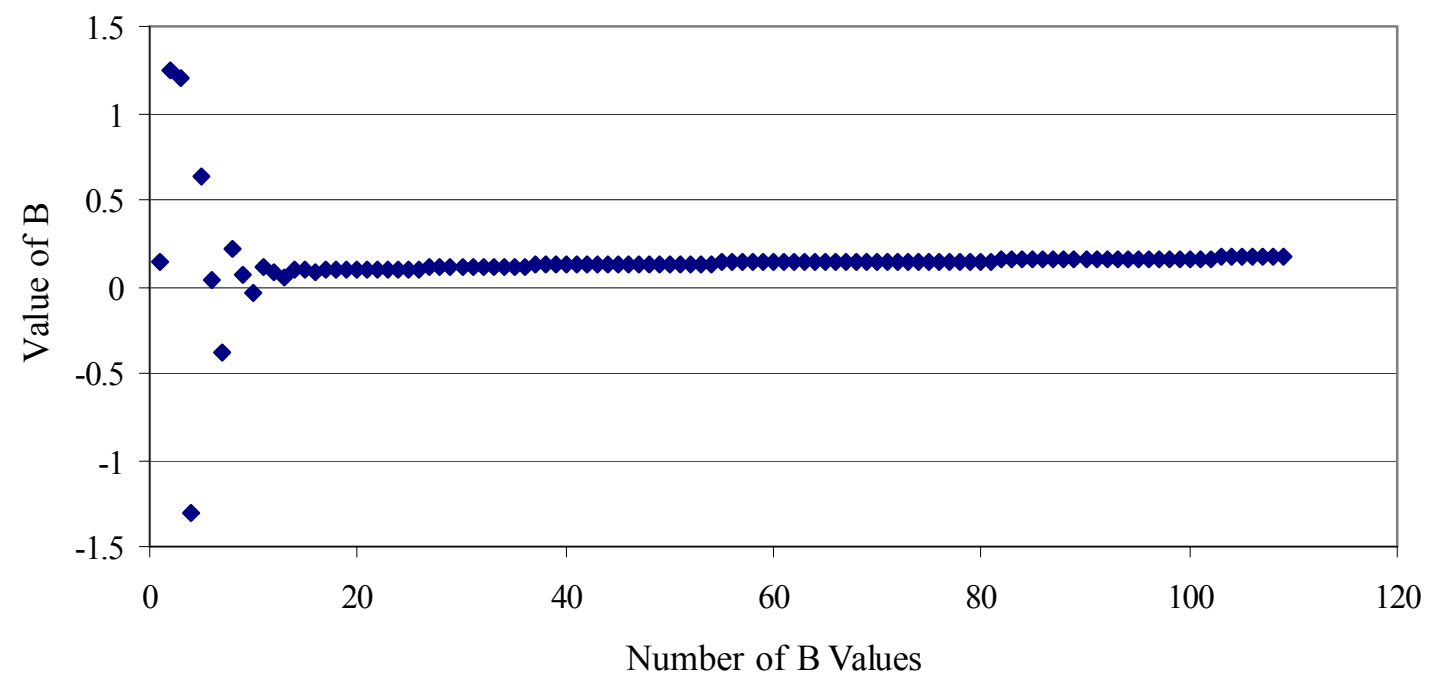

Figure D.43. ORF 1.3 
ORF 1.4

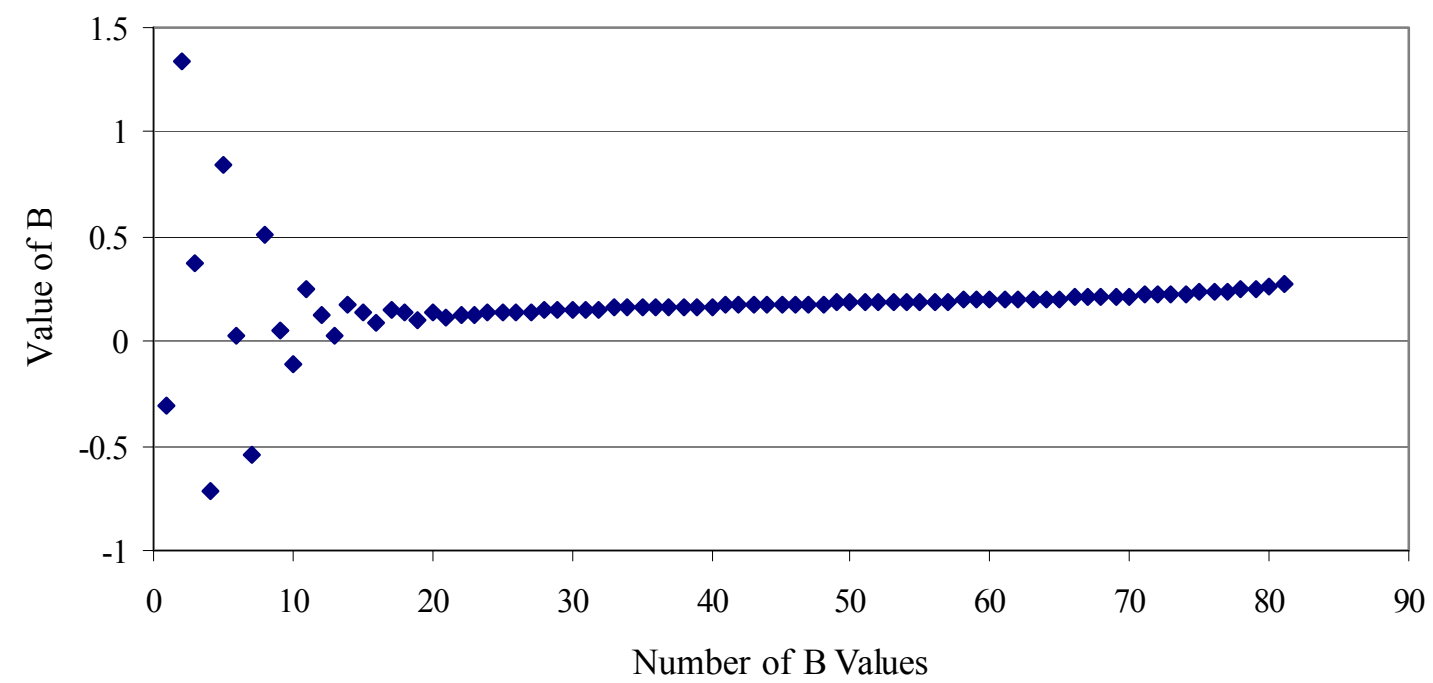

Figure D.44. ORF 1.4

ORF 1.5

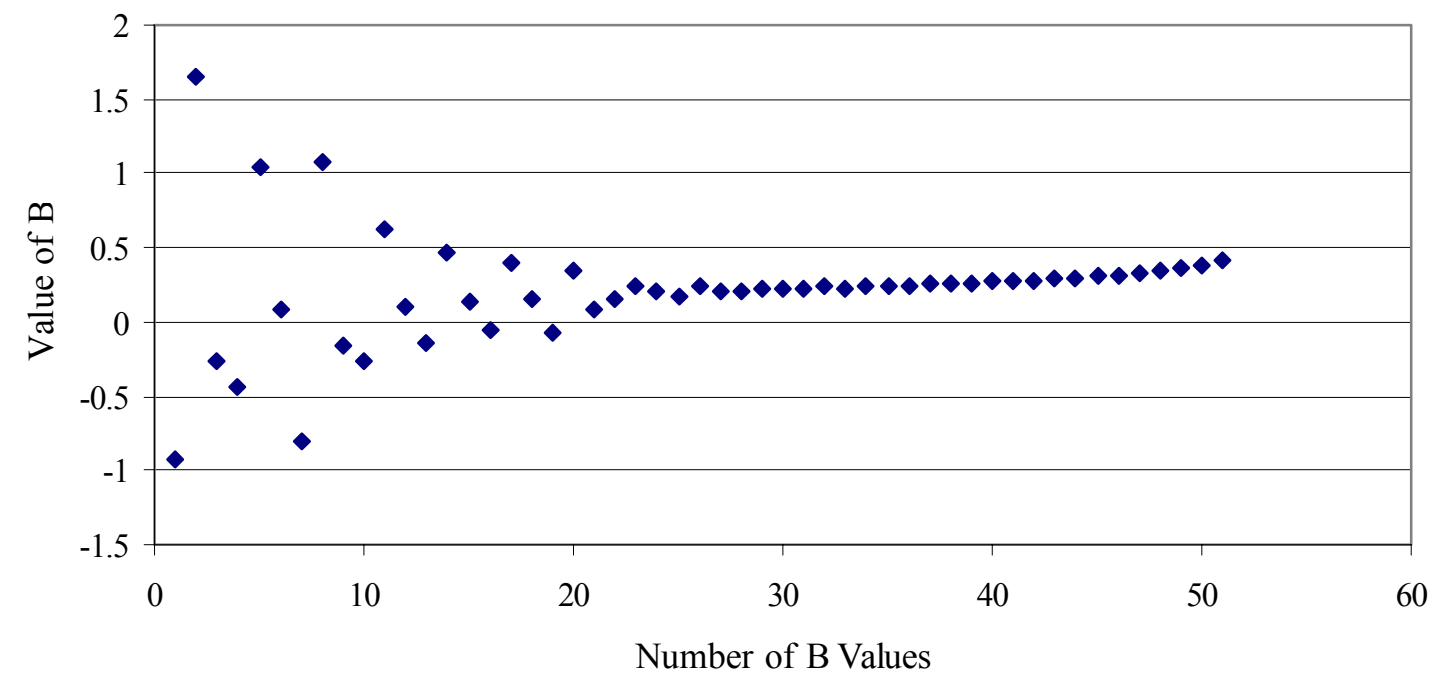

Figure D.45. ORF 1.5 
ORF 1.6

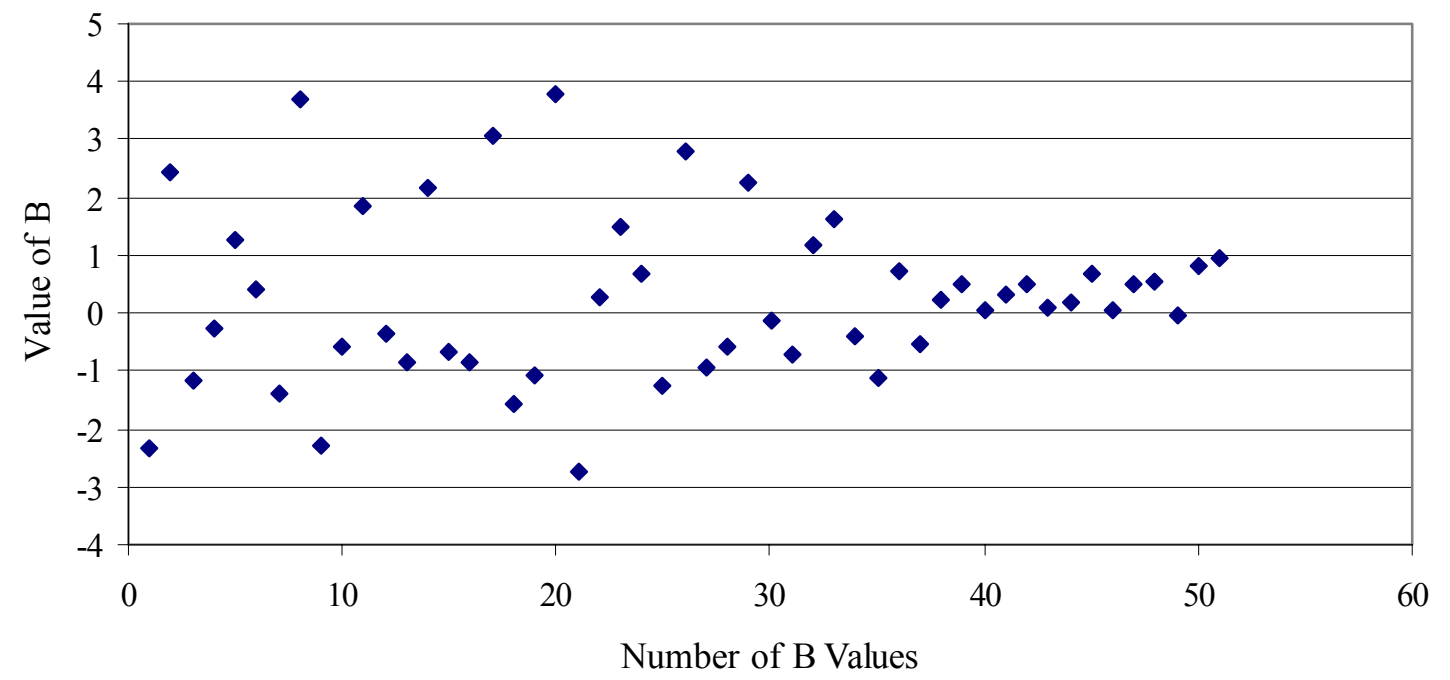

Figure D.46. ORF 1.6

\section{ORF 1.67}

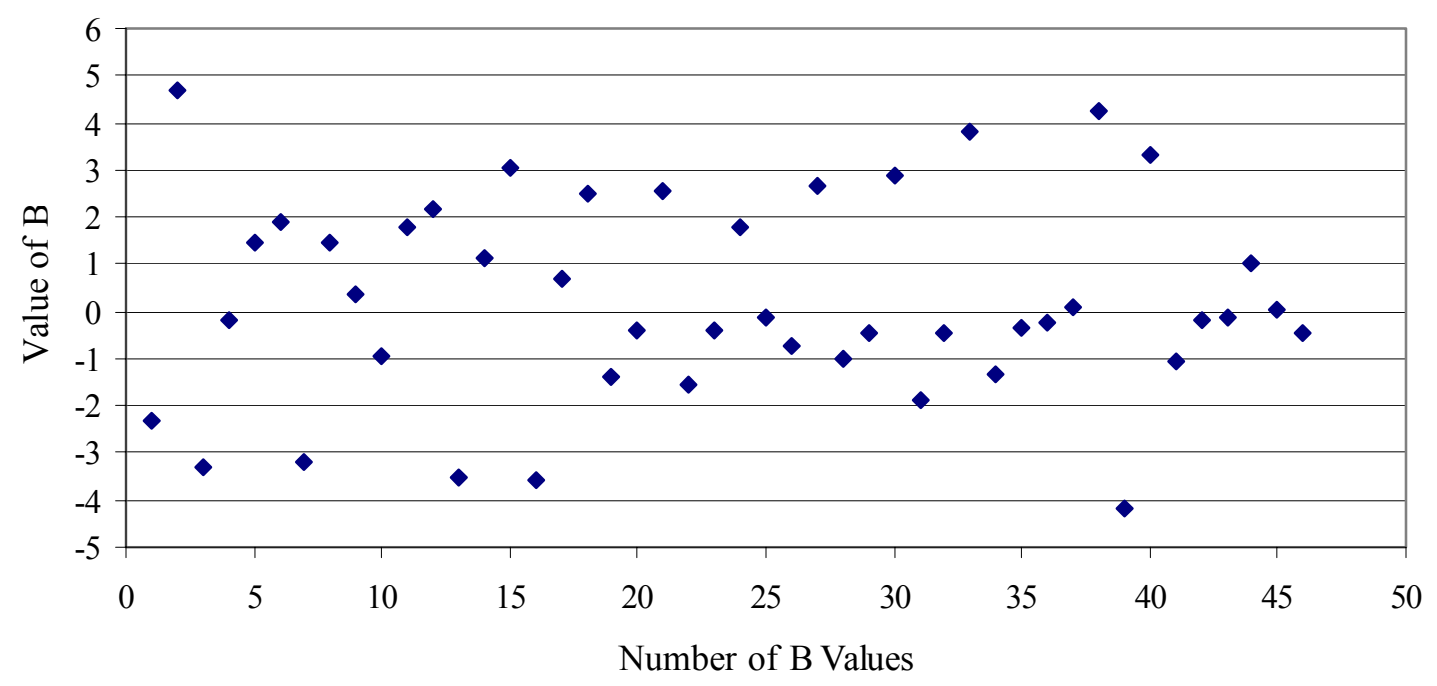

Figure D.47. ORF 1.67 


\section{ORF 1.678}

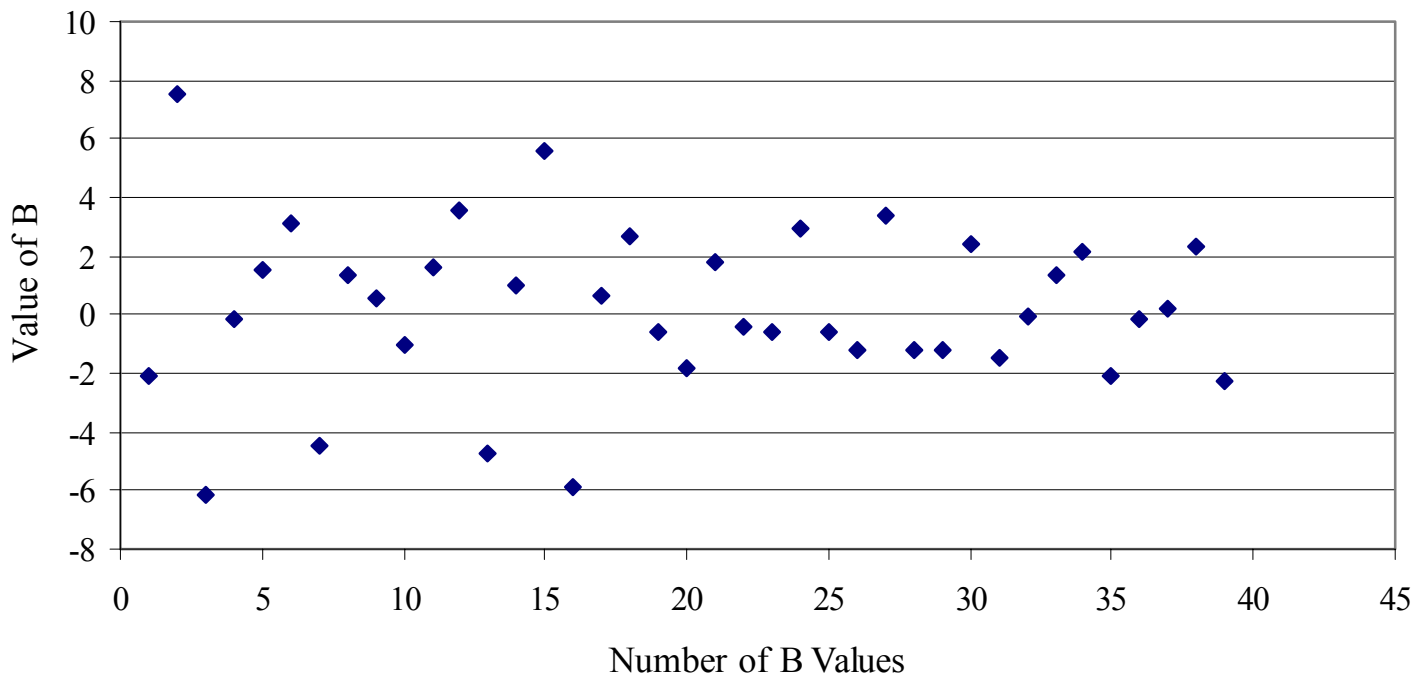

Figure D.48. ORF 1.678, $\omega_{\mathrm{opt}}$

\section{ORF 1.7}

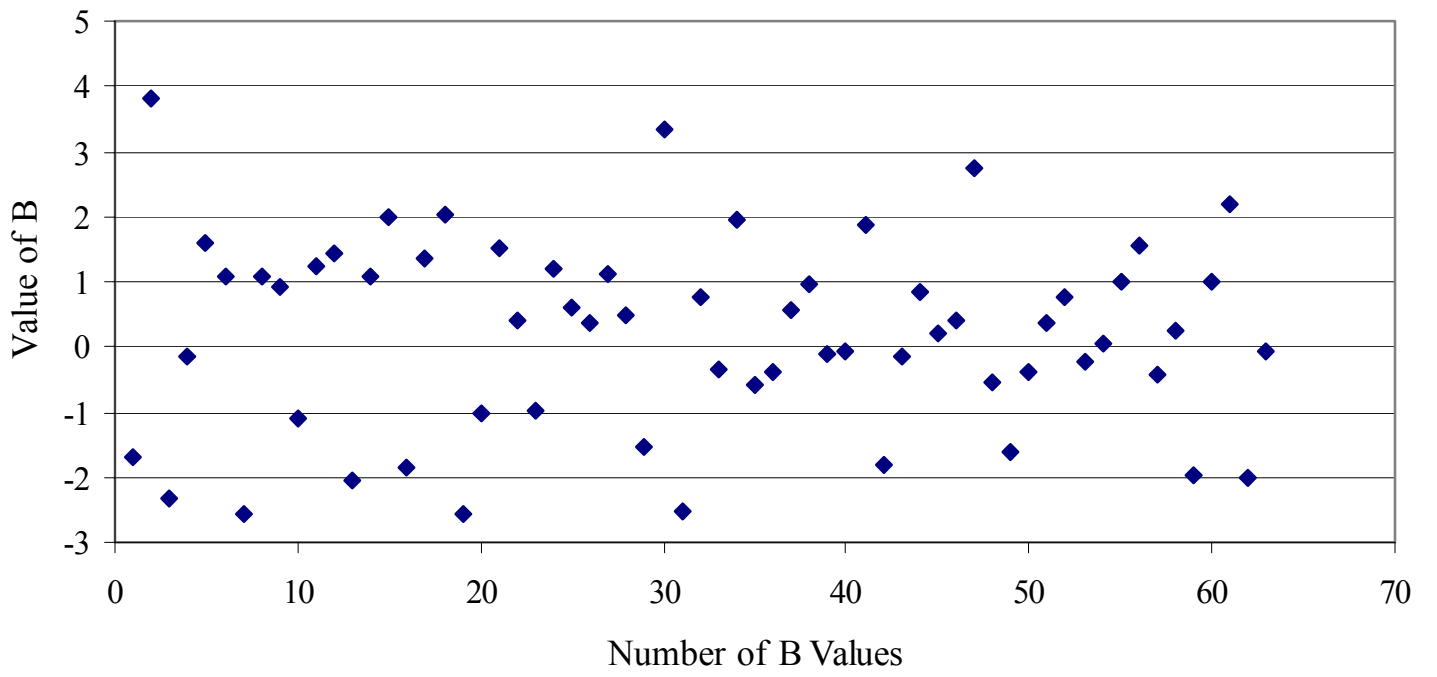

Figure D.49. ORF 1.7 
ORF 1.8

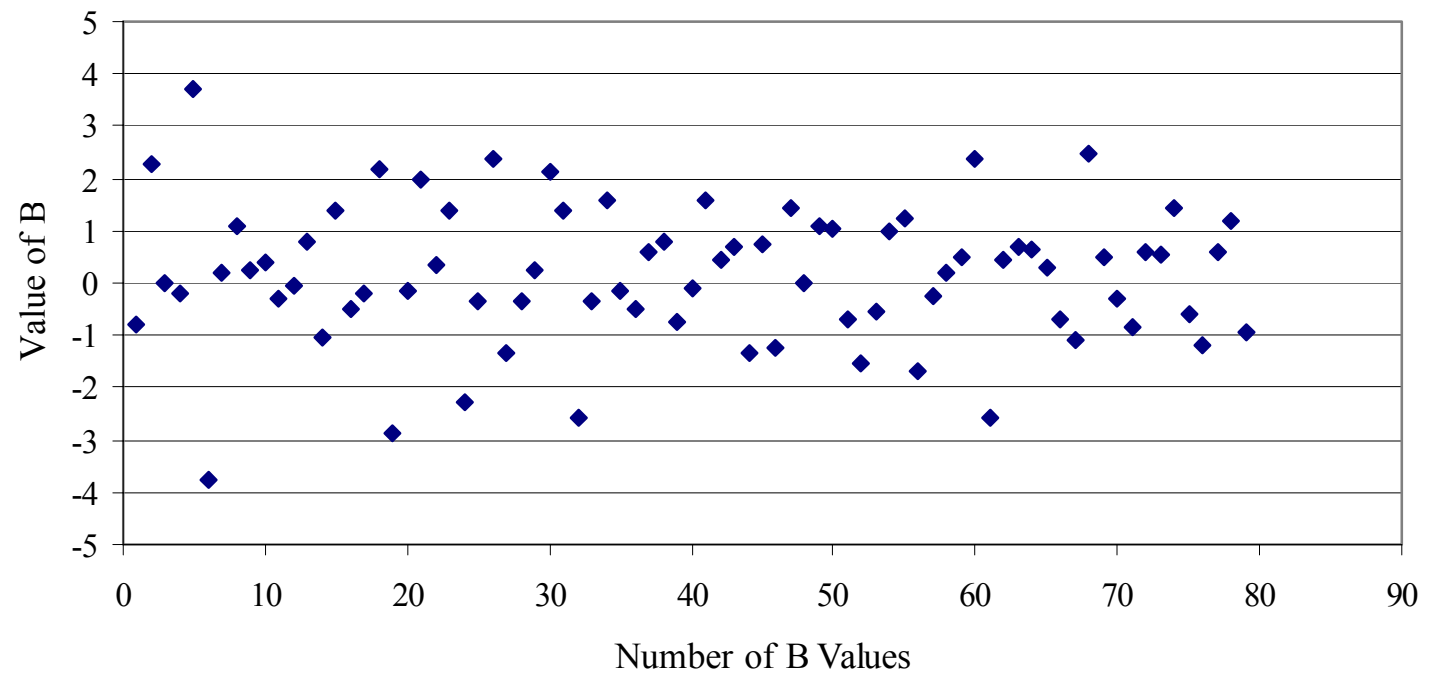

Figure D.50. ORF 1.8

ORF 1.9

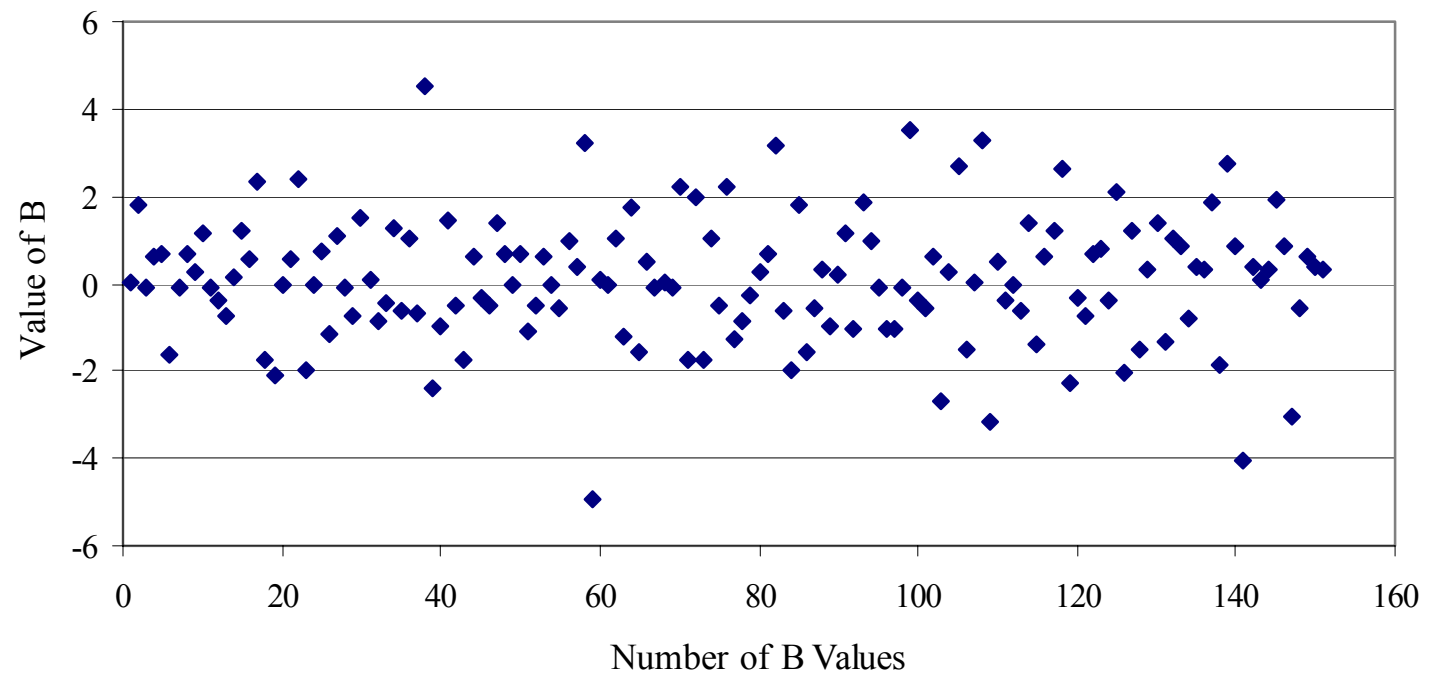

Figure D.51. ORF 1.9 
ORF 1.99

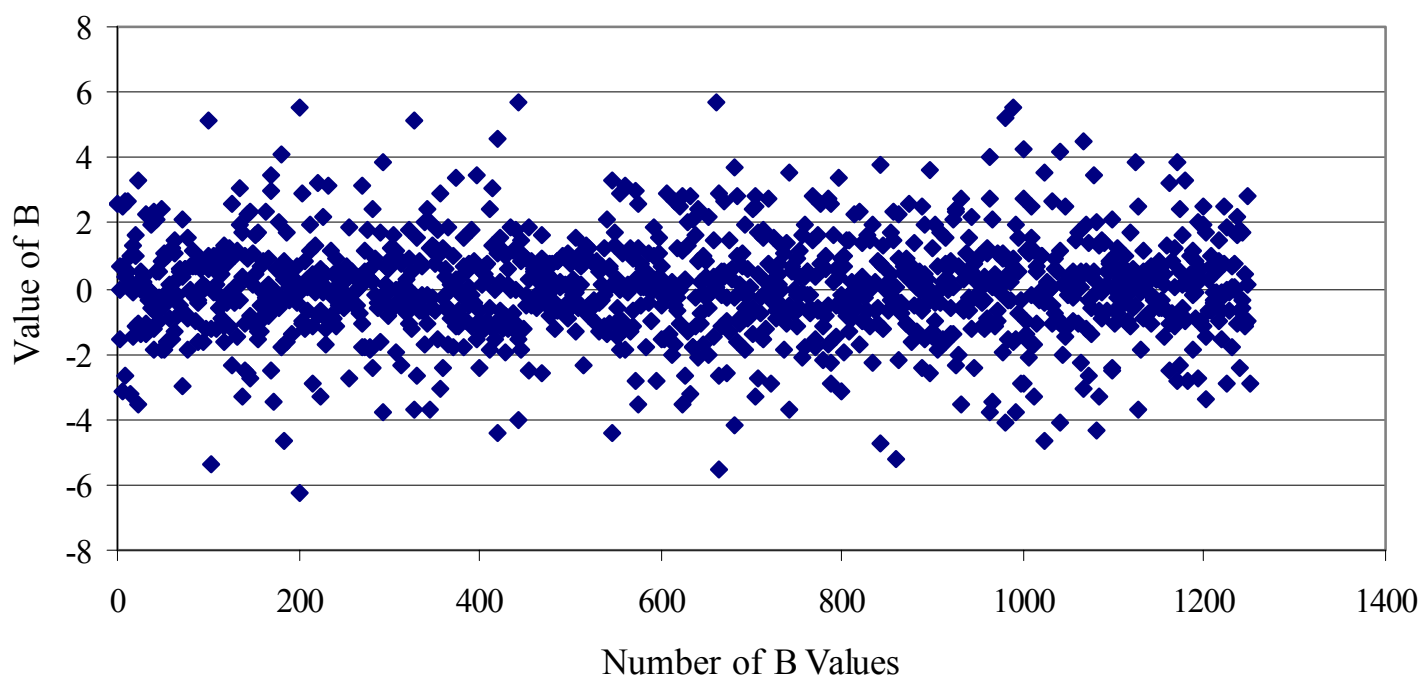

Figure D.52. ORF 1.99

These figures reflect the general trends that were observed with all nested-grid arrangements studied. For low over-relaxation factors, the values of $b$ initially oscillated during the first few iterations and then were suppressed to a near zero value for the remainder of the iterations. As the over-relaxation factor approached $\omega_{\text {opt }}$, the plots indicate an increase in the duration of the oscillatory nature of the $b$ value from the first iteration. At $\omega_{\mathrm{opt}}$ the $b$ value never was suppressed as previously observed with lower over-relaxation factors, but instead oscillated in value throughout the relaxation process. Over-relaxation factors larger than $\omega_{\text {opt }}$ showed continued and increased oscillation as the over-relaxation factor approached the unstable value of 2.0. These figures indicate that the rate of exponential decay of the error became essentially random around $\omega_{\mathrm{opt}}$; consequently, there appeared to be no effective way of determining $\omega_{\text {opt }}$ from a single run. 


\begin{abstract}
APPENDIX E
This Appendix contains a brief summary of all programs used in this thesis. As part of this summary, there is a brief description of things to pay attention to when running the programs. All working programs are contained on the CD that is included with this thesis. The programs are organized generally into Direct and Iterative Methods and the individual programs are found in corresponding folders.
\end{abstract}

\title{
DIRECT METHODS
}

\section{DGBSVREAD}

This folder contains the banded solver taken from the LAPACK library ${ }^{30}$ as well as a Visual Basic input generation file. The Visual Basic program LUARRAYIN generates a coefficient matrix input array. This input array is read into the Fortran program DGBSVREAD to obtain the pressure solution.

\section{Things to Consider: LUARRAYIN}

\section{LINES 11-13}

Set the dimensions of the reservoir.

$$
\begin{aligned}
& \operatorname{Imax}=11 \\
& \mathrm{Jmax}=11 \\
& \mathrm{Kmax}=22
\end{aligned}
$$

LINE 49

Make sure the proper output file is designated.

$$
\text { Open “E: Band11x11x22.dat” For Output as \#1 }
$$

When the output file has been correctly generated, open the file in notebook and make two corrections to the lines that have the well pressures of 1500 , and -1500 . The 
formatting distorts these two lines, and a space needs to be added so all columns are properly aligned. If this is not done the correct answer will not be generated when this input is used in the Fortran program DGBSVREAD.

\section{Things to Consider: DGBSVREAD}

\section{LINES 8,9}

Make sure the appropriate input file generated from Visual Basic program LUARRAYIN is used and that a desired output file is specified.

OPEN(1,FILE="D:/BAND11X11X22.dat")

OPEN(2,FILE="D:/ANSWER.txt")

LINES 11-13

Make sure the proper dimensions of the reservoir are entered.

$$
\begin{aligned}
& \operatorname{IMAX}=11 \\
& \mathrm{JMAX}=11 \\
& \text { KMAX }=22
\end{aligned}
$$

LINE 28

Make sure that the number following $L D A B$ is $=I M A X^{2}+1$. In this case $(11 x 11)$ $+1=122$.

$$
\text { READ(1,300),(AB(J,I),J=LDAB,122,-1),B(I) }
$$

\section{LINE 57}

This contains the output format so make sure a number equal to IMAX leads the formatting description. In this case the number is 11.

\section{$200 \quad$ FORMAT(11F16.3)}

\section{LINE 60}

Make sure that the first and third format specifications are led by the number $I M A X^{2}$. In this case the number is $11 \times 11=121$. 


\section{FEB22LU}

This folder contains the Doolittle LU decomposition as described in the book Numerical Methods for Scientists and Engineers. ${ }^{29}$

\section{Things to Consider: FEB22LU}

LINE 5

Make sure the Dimensions of the various arrays agree with the dimensions of grid being used. For example in this case $5 \times 5 \times 10=250$.

DIMENSION A(250,250),B(250),BP(250),X(250)

\section{LINES 11-13}

Make sure the proper grid dimensions are entered.

$\operatorname{IMAX}=5$

$\mathrm{JMAX}=5$

$\mathrm{KMAX}=10$ 


\section{SGAUSSELIM}

This folder contains the Gauss Elimination solution method. This program was taken from the book Numerical Methods for Scientists and Engineers. ${ }^{29}$

\section{Things to Consider: SGAUSSELIM}

LINE 6

Make sure the proper dimensions are allocated to each array. For example $5 \times 5 \times 10=250$.

DIMENSION A(250,250),B(250),X(250)

\section{LINE 12-14}

Make sure proper dimensions are entered.
$\operatorname{IMAX}=5$
$\mathrm{JMAX}=5$
$\mathrm{KMAX}=10$ 


\section{ITERATIVE METHODS}

\section{JACOBIMARO5}

This folder contains the Jacobi iterative method.

\section{Things to Consider: JACOBIMAR05}

LINE 12

Designate desired output file.

OPEN(1,FILE=DR//":/JACOBI.txt")

\section{LINES 14-16}

Make sure proper dimensions are entered.

$$
\begin{aligned}
& \text { IMAX }=5 \\
& \text { JMAX }=5 \\
& \text { KMAX }=10
\end{aligned}
$$

LINE 118

Make sure that the first number in the format description equals IMAX. For example IMAX is equal to 5 in this case.

200 FORMAT(5F16.6) 


\section{LSOR}

This folder contains the hybrid of Successive-Over-Relaxation and the tridiagonal solver (DGTSV) from LAPACK library. ${ }^{30}$ The Visual Basic program REVMEM provides the input file for the Fortran program SOVLVER3. To generate the input file from REVMEM, simply change the values of the desired dimensions and run the program. Before using the input file, open it in notepad and make sure that the lines containing the well pressures $1500,-1500$ are not out of line with the other columns. This input file is fed into the program SOLVER3 which takes care of the rest.

\section{Things to Consider: REVMEM}

\section{LINES 9-11}

Make sure the proper dimensions of desired grid are entered.

$$
\begin{aligned}
& \operatorname{Imax}=11 \\
& \operatorname{Jmax}=11 \\
& \operatorname{Kmax}=22
\end{aligned}
$$

LINE 54

Make sure proper output file is designated.

Open "e:\TDMA11.dat" For Output As \#1

\section{Things to Consider: SOLVER3}

\section{LINES 18,19}

Make sure that the proper input file is being used, as generated in Visual Basic REVMEM program, and that an output file is designated.

OPEN(1,FILE=DR1//":/TDMA11.dat")

OPEN(2,FILE=DR2//":/LSOR.txt")

\section{LINE 21-23}

Make sure the proper dimensions of the grid are entered

IMAX $=11$

JMAX $=11$

$\mathrm{KMAX}=22$ 
LINE 30

Make sure that the Optimal Over-Relaxation-Factor is being used.

$\mathrm{ORF}=1.8$

\section{LINE 156}

Make sure the output format is correct. The first number in the format description should be equal to IMAX.

200 FORMAT(11F16.6) 


\section{MATLAB}

The Fortran 90 programs in this folder are responsible for generating the $b$ vector that is used in MATLAB 7.0 to solve nested-grid arrangements. The Visual Basic programs generate the A matrix that is likewise used in MATLAB 7.0. The two input files generated from these programs are uploaded into MATLAB 7.0 and then the various solvers can be quickly and readily used to determine the solution. For help with MATLAB algorithms, simply use the HELP option.

Things to Consider: (These comments are based on MATLABREV1, but the same principles apply to the other Fortran programs)

\section{LINE 15}

Make sure the desired output file is in place.

OPEN(1,FILE=DR//":/MATREV5B1.txt")

LINE 17-19

Make sure that the proper dimensions are entered.

$$
\begin{aligned}
& \text { IMAX }=5 \\
& \text { JMAX }=5 \\
& \text { KMAX }=10
\end{aligned}
$$

LINE 21

For fastest time, enter in the Optimal Over-Relaxation-Factor.

$$
\mathrm{ORF}=1.988
$$

\section{LINE 172}

After this point the correct $b$ vector will be written to the output file and the rest of the code is not necessary, but simple recalculates the pressure solution using the nested grid points designated previously.

A similar pattern exits for all of the Fortran MATLAB programs

MATLABREV1: Generates input b vector with 18 Fixed Points

MATLABREV2: Generates input b vector with 130 Fixed Points

REV3MATLAB: Generates input b vector with 1026 Fixed Points

MATLABREV4: Generates input b vector with 8194 Fixed Points

MATLABREV5: Generates input b vector with 65538 Fixed Points 
Things to Consider: (These comments are based on SNOW2, but the same principles apply to the other Visual Basic programs)

LINE 24

Make sure proper output file is designated.

Open "E:\MAT17A3.dat" For Output As \#1

LINE 26-28

Make sure the correct grid dimensions are entered.

$$
\begin{aligned}
& \operatorname{Imax}=17 \\
& \mathrm{Jmax}=17 \\
& \mathrm{Kmax}=34
\end{aligned}
$$

LINES 105, 110, 120, 157,165, 171, 177, 185, 192, 198

Note the format of the output which indicates column and row indices as well as values of diagonal bands of input matrix.

Write \#1, COLIND, ROWIND, NNZ 


\section{LAPLACE_SOR}

This folder contains the files used in the LaPlace SOR nested-grid method.

\section{SOR}

The folder named SOR contains the Successive-Over-Relaxation algorithm that can be changed to Gauss-Seidel by setting the value of the Over-Relaxation-Factor to 1.0.

\section{Things to Consider: SOR}

LINE 13

Enter desired output file name. OPEN(1,FILE=DR//":/5X5X10.txt")

LINES 15-18

Enter the proper dimensions of the grid and the desired ORF value.

$$
\begin{aligned}
& \text { IMAX }=5 \\
& \text { JMAX }=5 \\
& \text { KMAX }=10 \\
& \text { ORF }=1.8
\end{aligned}
$$

LINE 32

Location in code where the convergence criterion can be adjusted.

\section{LINE 92}

Make sure leading number in format description equals IMAX. In the case shown, $I M A X=5$.

200 FORMAT(5F16.6) 


\section{AREV1, AVREV2, AREV3, AREV4, AREV5}

These folders contain programs that first calculate the entire fine-grid solution for a given grid size using SOR with only two wells. The initial part of the programs can be used to obtain solutions for the Gauss Seidel method (set ORF = 1.0) and for SOR (use optimal ORF value). The programs continue by determining the location of the fixed points for a given grid refinement and then nest the fixed pressures into the unsolved grid. The finelygridded pressure solution is then determined again by the nested-grid method.

$\begin{array}{ll}\text { AREV1 } & \text { 18 Fixed Points } \\ \text { AREV2 } & \text { 130 Fixed Points } \\ \text { AREV3 } & \text { 1026 Fixed Points } \\ \text { AREV4 } & \text { 8194 Fixed Points } \\ \text { AREV5 } & \text { 65538 Fixed Points }\end{array}$

Things to Consider: (These comments are based on AREV1, but the same principles apply to the other programs)

\section{LINE 15}

Make sure the desired output is designated

OPEN(1,FILE=DR//":/5X5X10.txt")

\section{LINE 17-20}

Make sure the proper dimensions for the grid are entered and that the desired value for ORF is set.

$$
\begin{aligned}
& \text { IMAX }=5 \\
& \text { JMAX }=5 \\
& \text { KMAX }=10 \\
& \text { ORF }=1.8
\end{aligned}
$$

\section{LINE 34}

The convergence criterion for SOR can be altered here.

\section{LINES 34-116}

Calculates and displays the SOR output of the fine-grid solution, which by this point in the code has been determined in its entirety.

\section{LINES 122-162}

This portion of the code designates the position of the fixed points in the nestedgrid configuration. 
LINE 169

Set the ORF value for the nested-grid calculation

$\mathrm{ORF}=1.5$

LINES 175-257

This portion of the code is the nested-grid algorithm and determines the finelygridded pressures. 


\section{FIXAREV, 17FIXAREV2, 17FIXAREV3, 17FIXAREV4, 17FIXAREV5}

These programs calculate the nested-grid solution by reading in desired nested-grid points for a specified level of grid refinement from a previously calculated fine-grid pressure solution. With the nested-grid configuration established, the fine-grid solution is determined.

To conduct a nested-grid study on the performance of LaPlace SOR, these are the fastest programs to run as the input has previously been generated using the SOR program.

$\begin{array}{ll}\text { 17FIXAREV } & \text { 18 Fixed Points } \\ \text { 17FIXAREV2 } & \text { 130 Fixed Points } \\ \text { 17FIXAREV3 } & \text { 1026 Fixed Points } \\ \text { 17FIXAREV4 } & \text { 8194 Fixed Points } \\ \text { 17FIXAREV5 } & \text { 65538 Fixed Points }\end{array}$

Things to Consider (These comments are based on 17FIXAREV, but same pattern applies to other programs)

LINE 15, 16

Make sure to designate the proper input and output files. Input files can be generated using the SOR program.

$$
\begin{aligned}
& \text { OPEN(1,FILE=DR//":/9X9X18.txt") } \\
& \text { OPEN(2,FILE=DR//":/9OUT.TXT") }
\end{aligned}
$$

LINES 18-21

Set the proper dimensions and Over-Relaxation-Factor

$$
\begin{aligned}
& \text { IMAX }=9 \\
& \text { JMAX }=9 \\
& \text { KMAX }=18 \\
& \text { ORF }=1.78
\end{aligned}
$$

LINE 31

Reads in the previously determined pressure values.

$\operatorname{READ}(1,201) \mathrm{P}$

\section{LINE 33,34}

Make sure the Input/Output format description agrees with the files being used. 
200 FORMAT(9F16.6)

201 FORMAT(9F16.9)

LINE 39-78

Sets the location of the fixed points in the nested grid configuration.

LINE 88-151

Calculation of the nested-grid solution. 


\section{WEBER}

This file contains all the programs used with Weber's coefficients in the nestedgrid method. The program HAWK3 was used to generate accurate pressures on a course grid. These pressures are then read into other programs that are made to handle a certain number of nested-grid points.

$\begin{array}{ll}\text { FALCONREV1 } & \text { 18 Fixed Points } \\ \text { FALCONREV2 } & \text { 54 Fixed Points } \\ \text { OSPREYREV3 } & \text { 130 Fixed Points } \\ \text { FALCONREV3 } & \text { 250 Fixed Points } \\ \text { OSPREYREV5 } & \text { 1026 Fixed Points } \\ \text { OSPREYREV6 } & \text { 6750 Fixed Points } \\ \text { HARRIER } & \text { 31250 Fixed Points }\end{array}$

HAWK3

Things to Consider: HAWK3

\section{LINES 3-25}

These lines allocate space required for various arrays used in this algorithm.

DOUBLE PRECISION, ALLOCATABLE :: OHMXP(:,:,:),OHMXM(:,:,:;)

DOUBLE PRECISION, ALLOCATABLE :: OHMYP(:,:,:),OHMYM(:,:,:)

DOUBLE PRECISION, ALLOCATABLE :: OHMZP(:,:,:),OHMZM(::,:,:)

OHMXP: solid angle for positive $x$ face of a given cell with respect to the first well.

OHMXM: solid angle for negative $x$ face of a given cell with respect to the first well.

Same nomenclature pattern applies in y and z directions.

DOUBLE PRECISION, ALLOCATABLE :: OHMXPT(:,:,:),OHMXMT(:,::;)

DOUBLE PRECISION, ALLOCATABLE :: OHMYPT(:,:,:),OHMYMT(:,:,:)

DOUBLE PRECISION, ALLOCATABLE :: OHMZPT(:,:,:),OHMZMT(:,:,:)

OHMXPT: solid angle for positive $x$ face of a given cell with respect to the second well.

OHMXMT: solid angle for negative $x$ face of a given cell with respect to the second well.

Same nomenclature pattern applies in y and z directions.

DOUBLE PRECISION, ALLOCATABLE :: RXP(:,:,:),RXM(:,:,:) 
DOUBLE PRECISION, ALLOCATABLE :: RYP(:,:,;),RYM(:,:,:;)

DOUBLE PRECISION, ALLOCATABLE :: RZP(:,:,:),RZM(:,:,:)

$\boldsymbol{R X P}$ : radial distance of a given point in positive direction from the first well.

$\mathbf{R X M}$ : radial distance of a given point in negative direction from the first well.

Same nomenclature pattern applies in the $y$ and $\mathrm{z}$ directions.

DOUBLE PRECISION, ALLOCATABLE :: RXPT(:,:,::),RXMT(:,:,:)

DOUBLE PRECISION, ALLOCATABLE :: RYPT(:,:,:),RYMT(:,:,:)

DOUBLE PRECISION, ALLOCATABLE :: RZPT(:,:,:),RZMT(:,:,:)

$\boldsymbol{R X P T}$ : radial distance of a given point in positive direction from the second well.

$\boldsymbol{R X M T}$ : radial distance of a given point in negative direction from the second well.

Same nomenclature pattern applies in the y and z directions.

DOUBLE PRECISION, ALLOCATABLE :: R(:,:::)

DOUBLE PRECISION, ALLOCATABLE :: RT(:,:::)

DOUBLE PRECISION, ALLOCATABLE :: P(:,:,:)

$\boldsymbol{R}$ : radial distance from the first well to any particular point.

$\boldsymbol{R T}$ : radial distance from the second well to any particular point.

$\boldsymbol{P}$ : array that contains pressure solution.

DOUBLE PRECISION, ALLOCATABLE ::

LAMBDAXP(:,:,:),LAMBDAXM(:,:,::)

DOUBLE PRECISION, ALLOCATABLE ::

LAMBDAYP(:,:,:),LAMBDAYM(:,:,:)

DOUBLE PRECISION, ALLOCATABLE ::

LAMBDAZM(:,:,:),LAMBDAZP(:,:,:)

LAMBDAXP: Lambda values for $\mathrm{x}$-face in positive direction

LAMBDAXM: Lambda values for $\mathrm{x}$-face in negative direction

Same nomenclature pattern applies in the $y$ and $\mathrm{z}$ directions.

LINE 40

Specify desired output file name.

OPEN(1,FILE=DR2//":/75NG.txt") 


\section{LINES 43-45}

These lines set the actual dimensions of the reservoir in feet.

$\mathrm{XLENGTH}=500$

YLENGTH=500

ZLENGTH=1000

LINES 48-51

These lines set the dimensions of the grid used as well as the Over-Relaxation-

Factor.

$\operatorname{IMAX}=5$

$\mathrm{JMAX}=5$

$\mathrm{KMAX}=10$

$\mathrm{ORF}=1.9831$

LINE 53-55

These lines specify variables that are used in the determination of the influence of Weber's coefficients as the grid is refined.

$\mathrm{CDIM}=5$

WS=IMAX/CDIM

WSS $=$ REAL(WS)

LINE 58-59

These lines allow you to specify the injection rate of the wells.

$\mathrm{Q} 1=250.0$

$\mathrm{Q} 2=-250.0$

LINE 61,62

Specify the format of output files.

200 FORMAT(5F16.9)

300 FORMAT(5F16.9)

LINE 65-74

These lines determine the physical size of the grid cells used and specify the ratio of the well radius to the length of a cell side. 
LINE 141-143

These lines specify the grid spacing.

LINE 149-481

Calculation of Weber's coefficients around the first well.

LINE 486-841

Calculation of Weber's coefficients around the second well.

LINE 886-939

Calculation of the finely-gridded pressure for the specified grid. 


\section{FALCONREV1}

This program is like HAWK3, but it does the nested-grid calculation using Weber's equations. The same patterns apply to all the other programs that use the nestedgrid method.

$\begin{array}{ll}\text { FALCONREV1 } & \text { 18 Fixed Points } \\ \text { FALCONREV2 } & \text { 54 Fixed Points } \\ \text { FALCONREV3 } & \text { 250 Fixed Points } \\ \text { OSPREYREV3 } & \text { 130 Fixed Points } \\ \text { OSPREYREV5 } & \text { 1026 Fixed Points } \\ \text { OSPREYREV6 } & \text { 6750 Fixed Points } \\ \text { HARRIER } & \text { 31250 Fixed Points }\end{array}$

Things to Consider: FALCONREV1 (Similar patterns are found in all remaining files found in the Weber folder)

LINE 27

These are the additional arrays needed to make the nested-grid method work.

DOUBLE PRECISION, ALLOCATABLE :: PP(:,:,:),DD(:,:,:),BP(:,:,::),VAL(:)

\section{LINES 32-33}

Additional parameters required for the nested-grid method.

$158-865$

Calculation of Weber's coefficients for both wells.

LINES 907-1070

These lines determine and fix the nested-grid points.

LINES 1098-1181

Determines the finely-gridded pressures using the nested-grid configuration. 


\section{REFERENCES}

1. U.S. Department of Energy, "Petroleum: An Energy Profile 1999”, Superintendent of Documents, U.S. Government Printing Office, P.O. Box 371954, Pittsburgh, PA 15250-7954, http://www.energy.gov/engine/content.do?BT_CODE=OIL.

2. Bundy, B.C.; Hales, H.B., "A Streamline Reservoir Simulator with Dynamic Gridding”, CIPC Paper 2004-303 presented at the Petroleum Society's $5^{\text {th }}$ Canadian International Petroleum Conference, Calgary, Alberta, Canada, June 8 - 10, 2004.

3. Erlandsen, S.M., "Production Experience From Smart Wells in the Oseberg Field", SPE Paper 62953 presented at the 2000 SPE Annual Technical Conference and Exhibition held in Dallas, Texas, 1-4 October 2000.

4. Barker, J.W.; Thibeau, S., "A Critical Review of the Use of Psuedorelative Permeabilities for Upscaling”, SPE Reservoir Engineering, 12, May 1997, pp. 138143

5. Christie, M.A., "Flow in Porous Media-Scale Up of Multiphase Flow", Current Opinion in Colloid \& Interface Science, 6 (3): 236-241 Jun 2001.

6. Evans, S., "Reservoir Modeling and Simulation in Today's High-Performance Computing Environments”, Oil and Gas Journal, May 17 2004, p 33-37.

7. Christie, M.A.; Blunt, M.J., "Tenth SPE Comparative Solution Project: A Comparison of Upscaling Techniques”, SPE Reservoir Evaluation \& Engineering, 4, August 2001, pp. 308-317.

8. Durlofsky, L.J., "Numerical Calculation of Equivalent Grid Block Permeability Tensors for Heterogeneous Porous Media”, Water Resources Research, May 1991,Vol 27, No. 5, pp. 699-708.

9. Durlofsky, L.J.; Milliken, W.J.; Bernath, A., "Scaleup in the Near-Well Region", SPE Journal 5 (2000), pp. 110-117.

10. Efendiev, Y.; Durlofsky, L.J.; Lee S.H., "Modeling of Subgrid Effects in Coarse Scale Simulations of Transport in Heterogeneous Porous Media”, Water Resources Research, Vol. 36, No. 8, pp. 2031-2041, August 2000. 
11. Efendiev, Y.; Durlofsky, L.J., "Numerical Modeling of Subgrid Heterogeneity in Two Phase Flow Simulations”, Water Resources Research, Vol. 38, No. 8, 3-1-3-11, 2002.

12. Guedes, S.S.; Petrobras; Schiozer, D.J, “An Implicit Treatment of Upscaling in Numerical Reservoir Simulation”, SPE Symposium on Reservoir Simulation, SPE 51937, Houston, 14-17 February, 1999.

13. Christie, M.A., "Upscaling for Reservoir Simulation”, Journal of Petroleum Technology, Nov 1996, 48 (11), p 1004-1010.

14. Farmer, C.L., “Upscaling: a Review”, International Journal for Numerical Methods in Fluids, 2002, 40: 63-78.

15. Renard Ph, de Marsily G., “Calculating Equivalent Permeability: a Review”, Advances in Water Reserouces, Vol. 20, pp. 253-278, 1997.

16. Wen, X.H.; Gomez-Hernandez, J. J., “Upscaling Hydraulic Conductivities in Heterogeneous Media: An overview”, Journal of Hydrology 183 (1996) ix-xxxii.

17. Audigane, P.; Blunt, M.J., "Dual Mesh Method for Upscaling in Waterflood Simulation”, Transport in Porous Media 55: 71-89, 2004.

18. Ramé, M.; Killough, J.E., “A New Approach to the Simulation of Flows in Highly Heterogeneous Porous Media”, SPE Symposium on Reservoir Simulation, SPE No. 21247, Anaheim California 11-17 February 1991.

19. Guérillot, D.; Verdière, S., "Different Pressure Grids for Reservoir Simulation in Heterogeneous Reservoirs, SPE Symposium on Reservoir Simulation, SPE No. 29148 San-Antonio, 12-15 February 1995.

20. Verdière, S.; Guérillot, D., "Dual Mesh Method for Multiphase Flows in Heterogeneous Media, 5th European Conference on the Mathematics of Oil Recovery, Leoben, Austria, 3-6 September 1996.

21. Hou, T.Y.; Wu, X.H., “A Multiscale Finite Element Method for Elliptical Problems in Composite Materials and Porous Media”, Journal of Computational Physics 134, 169-189, 1997.

22. Guedes, S.S.; Schiozer, D.J., “An Implicit Treatment of Upscaling in Numerical Reservoir Simulation”, SPE Symposium on Reservoir Simulation, SPE 51937, Houston, 14-17 February 1999.

23. Hermitte, T.; Guerillot, D., “A More Accurate Numerical Scheme for Locally Refined Meshes in Heterogeneous Reservoirs”, SPE Symposium on Reservoir Simulation, SPE No. 25261, New Orleans, 28 February -3 March. 1995. 
24. Gautier, Y.; Blunt, M.J.; Christie, M.A., "Nested Gridding and Streamline-Based Simulation for Fast Reservoir Performance Prediction", Proceedings of the SPE Symposium on Reservoir Simulation, 1999, SPE 51931, p 403-412.

25. Arbogast, T.; Bryant, S.L, "Numerical Subgrid Upscaling for Waterflood Simulations”, SPE Reservoir Simulation Symposium, SPE No. 66375, Houston, 1114 February 2001.

26. Peaceman, D.W., “Fundamentals of Numerical Reservoir Simulation”, Elsevier Scientific Publishing Company, New York, 1977.

27. Weber, D.B.; Hales, H.B.; L.L. Baxter, “A New Method of Formulating Finitedifference Equations -- Some Reservoir Simulation Examples”, CIPC Paper 2004-170 presented at the Petroleum Society's $5^{\text {th }}$ Canadian International Petroleum Conference, Calgary, Alberta, Canada, June 8 - 10, 2004.

28. Briggs, W.L., “A Multigrid Tutorial”, Society for Industrial and Applied Mathematics, Philadelphia, Pensylvania, 1987.

29. Hoffman, J.D., "Numerical Methods for Engineers and Scientists”, Second Edition, Marcel Dekker, Inc., New York, 2001.

30. Anderson, E.; Bai, Z.; Bischof, C.; Blackford, S.; Demmel, J.; Dongarra, J.; Du Croz, J.; Greenbaum, A.; Hammarling, S.; Mckenney, A.; Sorsensen, S., "LAPACK Users' Guide; Third Edition”, SIAM, Philadelphia, PA, 1999.

31. Barrett, R.; Berry, M.; Chan, T.F.; Demmel, J.; Donato, J.; Dongarra, J.; Eijkhout, V.; Pozo, R.;Romine, C.; Vorst, V., "Templates for the Solution of Linear Systems: Building Blocks for Iterative Methods”, 2nd Edition, SIAM, Philadelphia, PA, 1994.

32. Mathworks, Inc., MATLAB 7.0 The Language of Technical Computing; Mathworks www.mathworks.com.

33. Hardy, B.A.; Hales, H.B.; Baxter, L.L, "A New Method for the Rapid Calculation of Finely-Gridded Reservoir Simulation Pressures”, CIPC Paper 2005-112 presented at the Petroleum Society's $6^{\text {th }}$ Canadian International Petroleum Conference, Calgary, Alberta, Canada, June 7-9 2005.

34. Flannery, B.P.; Press, W.H; Teukolsky, S.A.; Vetterling, W.T., "Numerical Recipes in Fortran 77"; Second Edition, Press Syndicate of the University of Cambridge, New York, 1992. 
